\title{
COMPONENTS OF NEGATIVE EMOTION AND EMOTION-CONGRUENT INFORMATION PROCESSING BIASES
}

by

Carl John Beuke

\author{
A thesis \\ submitted to Victoria University of Wellington \\ in fulfilment of the \\ requirements for the degree of \\ Doctor of Philosophy \\ in Psychology
}

Victoria University of Wellington

2002 


\section{Acknowledgements}

I thank those who have, in a variety of ways, helped to make this thesis possible. First, my supervisor, Dr John McDowall, has provided good-natured guidance and invaluable support. Thank you. Second, my thanks to the School of Psychology’s support staff and to the students and academic staff who took responsibility for arranging the student research participation program and the mass testing sessions. Your work made my thesis much easier than it would have been: thank you Ngaire Lavery, Jaana Salo, Stephanie Simpson, Keith Riach, Douglas Flux, Gary Jowett, Dr Todd Jones, Dr Marc Wilson, Stefanie Sharman and Dr Maryanne Garry. Thank you to the participants that made this research possible. My thanks also to the researchers from other universities who supplied advice or additional details of their procedures and stimuli. Thank you to the School's vibrant postgraduate community. In particular, thanks to Joseph Oliver and Angela Mansell for your advice, your moral support, and your interest in my statistical procedures. Extra thanks to Caroline Fisher and Kristy Bolter for having the patience to carefully read my entire thesis; I am most grateful for your feedback. Thank you to my parents for your lifelong support and encouragement. I acknowledge the generous financial support of the Foundation for Research, Science, and Technology. Finally, my thanks to all those who helped, but whom I have not mentioned by name. 


\begin{tabular}{|c|c|}
\hline & \\
\hline Abstract & 1 \\
\hline Introduction & 2 \\
\hline Methodological issues & \\
\hline Anxiety and depression & 6 \\
\hline Clinical and nonclinical samples & 7 \\
\hline Statistical power & 11 \\
\hline Mood and memory & \\
\hline Autobiographical memory & 13 \\
\hline Nonautobiographical memory & 20 \\
\hline Mood and attention & 27 \\
\hline Mood and judgement & 35 \\
\hline Theories of information processing in anxiety and depression & 42 \\
\hline Somatic and cognitive aspects of depression and cognitive biases & 47 \\
\hline Using information processing biases to measure depression & 49 \\
\hline Hemispheric thinking style and information processing & 50 \\
\hline The purpose of the current investigation & 51 \\
\hline Experiment 1 & \\
\hline Method & 57 \\
\hline Results & 64 \\
\hline Discussion & 84 \\
\hline Experiment 2 & \\
\hline Method & 92 \\
\hline Results & 101 \\
\hline Discussion & 124 \\
\hline General discussion & \\
\hline Implications of findings relevant to Aim 1 & 127 \\
\hline Implications of findings relevant to Aim 2 & 134 \\
\hline Implications of findings relevant to Subsidiary Aim 1 & 138 \\
\hline Implications of findings relevant to Subsidiary Aim 2 & 138 \\
\hline Implications of findings relevant to Subsidiary Aim 3 & 141 \\
\hline Implications of findings relevant to Subsidiary Aim 4 & 142 \\
\hline Implications of the overall project & 143 \\
\hline References & 151 \\
\hline
\end{tabular}




\begin{abstract}
Negative emotion is often associated with emotion-congruent biases in information processing. However, rather than all negative emotion being associated with biases in all information processes, certain components of emotion appear to be associated with specific biases. This project examined two examples of specific associations. First, Williams, Watts, MacLeod, \& Mathews $(1988,1997)$ have argued that anxiety is associated with biases on tasks involving priming, and depression is associated with biases on tasks involving elaboration. Second, most models of mood-congruent bias have given purely cognitive explanations; these models suggest that biases should be more closely associated with the cognitive symptoms than the somatic symptoms of depression (Horowitz, Nelson, \& Person, 1997). Evidence is reviewed that suggests this may not be the case. These issues were examined in two experiments, each of which administered a broad range of tasks to a large sample of students. The experiments examined attention and judgement, and explicit, implicit, and autobiographical memory. It was hypothesised that Williams et al.'s $(1988,1997)$ predictions about the task-specific effects of anxiety and depression would be confirmed, and that the somatic symptoms of depression would have a greater influence on information processing biases than the cognitive symptoms. Emotion-congruent biases were not shown on every task, but on the tasks where biases were shown, the hypotheses were broadly confirmed. Strengths, limitations, and implications of the studies are discussed. Current cognitive and neuropsychological models of emotion are used to provide a possible explanation of the results.
\end{abstract}


Information processing is the selection, transformation, storage, and use of information obtained from the senses. Emotion-related information processing biases occur when preferential treatment is given by the information processing system to material with specific emotional content. Biases in attention occur when either positive or negative information is preferentially processed by the system. Biases in memory occur when either positive or negative material is preferentially stored or recalled. Biases in judgement occur when ambiguous material is preferentially interpreted as either negative or positive.

Interest in emotionally biased information processing arose out of the empirical investigation of Beck’s cognitive model of depression (Beck, Rush, Shaw, \& Emery, 1979). At the time that Beck introduced his model, the two dominant models of psychotherapy were Freudian and behavioural (Hunt, 1993). The Freudian tradition did not prove receptive to experimental investigations (Macmillan, 1991), and the behavioural paradigm was more concerned with external influences on behaviour than on the internal mental state of individuals (Beidel \& Turner, 1986). In contrast, the cognitive model placed high importance on the thoughts and information processing of individuals, and the model has inspired a remarkable quantity of research (Clark, Beck, \& Alford, 1999). Although the cognitive model was first developed as a model of depression, it has since been extended to model many other mental disorders and difficulties, particularly anxiety (Clark et al., 1999).

Early research seemed to raise some challenges to the cognitive model. Beidel and Turner (1986) argued strongly that the cognitive model of depression was not supported by sufficient empirical evidence. They argued that though both behaviourists and cognitivists accept that cognition is a "description of 
antecedent and consequent events” (Beidel \& Turner, 1986, p. 313), these groups of theorists disagree about the causal nature of cognition. Cognitive theory postulates that cognition actively mediates the relationship between antecedent stimuli (what happens to the individual) and the individual's resulting emotions and behaviours. In behavioural theory, thoughts “merely describe the relationship between antecedent events and their contingencies” (Beidel \& Turner, 1986, p. 313). Beidel and Turner (1986) argued that both behaviour itself, and thoughts and emotions, which they conceptualise as private behaviour, are fully determined by the known laws of learning. Presumably, by the known laws of learning they mean classical and operant conditioning, and perhaps modelling.

Beck’s cognitive theory has always been lent credibility by the success of the therapies that it inspired. However, Beidel and Turner (1986) pointed out that the cognitive and cognitive-behavioural therapy treatment plans that existed at that time all contained extensive use of known behavioural techniques. Thus, it was not possible to determine whether the effectiveness of cognitive treatments was due to the use of cognitive components, or was attributable to the behavioural components alone. Although more recently therapists have developed successful treatment plans they claim are purely based on the cognitive model (Free, 1999), it is possible to explain the mechanism of almost any plausible treatment plan in terms of the behavioural model, just as it is possible to explain the mechanism of behavioural treatments in terms of the cognitive model (Lilienfeld, 1995). Beidel and Turner (1986) also argued that treatments classified as cognitive-behavioural are no more effective than treatments classified as purely behavioural. Although purely behavioural treatment is uncommon in current clinical practice, the conclusion of equal 
effectiveness remains consistent with more recent evidence on the treatment of depression (Jacobson, Martell, \& Dimidjian, 2001) and the anxiety disorders

(Feske \& Chambless, 1995; Sweet \& Loizeaux, 1991; van Blakom et al., 1994).

Thus, the final evaluation of the cognitive model requires theoretically oriented research. One critical research strategy is to investigate cognitive variables and examine whether they predict later psychopathology. Researchers have often chosen depression as the disorder of interest in these experiments. Beidel and Turner (1986) reviewed the relatively small literature that existed at that time, and found that measures of negative or purportedly irrational cognitive content correlate with depression, but do not predict it. At that time, a few studies of cognitive process had been conducted, but these studies examined only the association of cognitive processes with depression, not the causal direction of the relationship. Thus, Beidel and Turner (1986) concluded that negative cognitions are a correlate of depression, but that there was no evidence to show that they caused depression. ${ }^{1}$

In a more recent review, Gotlib and Krasnoperova (1998) found that most studies have shown that cognitive content does not predict later depression. However, these authors also reviewed evidence that cognitive processing is more negative in depression than in normal mood states. More importantly, they

\footnotetext{
${ }^{1}$ It may come as a surprise to some that Beck has also explicitly stated that he does not believe that cognition causes depression (Beck \& Steer, 1991). Rather, negative thoughts are conceptualised as an integral part of depression; "To conclude that cognitions cause depression is analogous to asserting that delusions cause schizophrenia” (p. 371). The theory holds that the activation of latent maladaptive schemas is the proximal mechanism of the development of depression, and thus the presence of such schemas represents a major vulnerability factor (Clark et al., 1999; schemas are defined later in this thesis). The distinction between a vulnerability factor and a causal factor that is not, in itself, necessary and sufficient to cause depression is not a clear one; suffice to say that evidence that can be used to support the existence of one can also be used to support the existence of the other. Clark et al. (1999) claim that Beck's theory has often been misunderstood, but it would perhaps be more accurate to say that, especially in it's early formulations, the theory was ambiguous about some issues (Williams et al., 1997), particularly the distinction between cognitive content and cognitive processes (Beidel \& Turner, 1986).
} 
reviewed several studies that suggested that negative cognitive processing may play a causal role in depression. Two types of evidence for causality exist. First, studies have examined whether negative biases in processing predict later depression. Second, and more commonly, studies have examined whether individuals who are known to be vulnerable to depression (for example, the previously depressed) show negative biases in cognitive processing. The issue of causality will be returned to in the discussion.

It is partly because of the possible role of cognitive biases in the aetiology of anxiety and depression that these processes have become a focus of research interest. Most research has focussed on identifying cognitive biases that co-occur with anxiety and depression; the existence of such biases must first be determined before causality can be investigated. Cognitive biases are also a subject of interest in their own right, because even if they are a consequence of depression rather than a cause, they may help illuminate the functions of anxiety and depression (Mathews, 1990). In addition, Horowitz, Nelson, and Person (1997) suggest using a battery of measures of cognitive bias as an instrument for assessing level of depression. These possibilities are further explored later in this thesis.

This introduction reviews the literature on cognitive biases in information processing. First, there is a discussion of the main methodological issues that affect research on cognitive biases. Then there is a review of the way that emotional information is selectively attended to, interpreted, and stored in and retrieved from memory. Finally, there is a discussion of the way that Beck's cognitive theory explains biases in these processes, and of some competing 
theories. This discussion leads into an explanation of the purposes and hypotheses of the current studies.

\section{$\underline{\text { Methodological Issues }}$}

Anxiety and depression. Anxiety and depression are highly associated in both unselected and clinical populations (Clark \& Watson, 1991a).

Consequently, it has been suggested that studying their separate effects is meaningless. Ingram and Hamilton (1999) discount this suggestion, arguing that the stated intention of investigators is usually to investigate anxiety or depression, not general negative affect. Consistent with this argument, anxiety and depression are generally regarded as distinct enough to warrant separate review (Williams et al., 1997), and studies of depression have been reviewed without mentioning anxiety at all (Gotlib \& Krasnoperova, 1998). These reviews have found that despite their close association, anxiety and depression are associated with different aspects of information processing. As discussed later in this introduction, these differences are of particular interest in the evaluation of different models of information processing biases. In addition, the failure to consider the potential effects of the high degree of association between anxiety and depression represents an important flaw in many studies of both emotional states. This issue will be discussed as appropriate in the relevant sections. Finally, many existing studies that have measured both anxiety and depression have measured anxiety using the Spielberger State-Trait Anxiety Inventory (STAI; Spielberger, Gorsuch, Lushene, Vagg, \& Jacobs, 1983). Unfortunately, this measure is not well suited to differentiating the effects of anxiety from those 
of depression. Bieling, Antony, and Swinson (1998) found that the STAI-trait scale contains a factor that appears to measure depression rather than anxiety, consisting of items like "I am happy” (reverse scored) and "I feel like a failure”. Although Bieling et al. (1998) did not examine the state scale, this also contains items that appear to reflect depression rather than anxiety (for example, "I feel satisfied”, “I feel content”, both reverse-scored). Endler, Cox, Parker, and Bagby (1992) found that neither scale of the STAI successfully differentiated anxiety from depression, but that the Endler Multidimensional Anxiety Scales (EMAS) did differentiate them. Similarly, Kennedy, Schwab, Morris, and Beldia (2001) found that neither scale of the STAI differentiated anxiety disorders from clinical depression. A primary purpose of the current investigation is to compare the effects of anxiety and depression across a variety of tasks, using measures that clearly discriminate between the two emotions.

\section{Clinical and nonclinical samples. Research into anxiety and depression} has relied primarily on two kinds of studies. The first kind of study compares participants who meet the DSM-IV criteria for a mood or anxiety disorder (American Psychiatric Association, 1994) to community controls. Participants who meet DSM-IV criteria are diagnosed using a structured or unstructured interview, and are often referred to as clinically anxious or depressed. The second kind of study compares participants with high scores on self-report inventories to participants with low scores. High-scoring individuals are often referred to as subclinically anxious or depressed, and studies that compare subclinical to nondistressed samples are often called nonclinical studies. Some members of subclinical samples may be clinically depressed or anxious, or even 
undergoing treatment for their emotional distress, but investigators who use these samples do not generally have information about this. The use of subclinical samples has been the subject of recent debate in the literature (Coyne, 1994; Flett, Vredenburg, \& Krames, 1997; Vredenburg, Flett, \& Krames, 1993). One often-overlooked problem is that control participants in studies of subclinical depression are often extremely low scorers on self-report inventories measuring psychological distress, and this can potentially lead to artifactual results (Ingram \& Hamilton, 1999). However, it is not altogether clear that studies of clinical depression always avoid this problem. The experiments reported in this thesis endeavour to use representative control groups. Coyne (1994) also points out that the distinction between clinical and nonclinical studies is often not made clear. The literature review that follows avoids this problem by clearly labelling studies as either clinical or nonclinical. The terms subclinically anxious and subclinically depressed are used in preference to the term dysphoria, because the latter is often taken to imply generalised distress, without distinguishing between anxiety and depression. As will become clear, there are important differences between the effects of subclinical anxiety and subclinical depression.

The continuity debate in depression has been a focus of much recent research attention. If depression is continuous, then subclinically depressed individuals are similar to clinically depressed individuals, but less extreme. If depression is discontinuous, then clinical depression is a qualitatively different phenomenon from subclinical depression. Coyne and Schwenk (1997) argue that subclinical depression, which they call “distress”, is associated with little impairment. However, every other major empirical study that has directly addressed this question has shown that subclinical and clinical depression are 
continuous on most of the attributes investigated, and that subclinical depression is a condition with serious consequences for the lives of those affected by it (Cox, Enns, Borger, \& Parker, 1999; Gotlib, Lewinsohn, \& Seeley, 1995; Lewinsohn, Solomon, Seeley, \& Zeiss, 2000; Ruscio \& Ruscio, 2000). The continuity of anxiety appears to have been a relatively neglected topic, perhaps because the issue is complicated by the assortment of different anxiety disorders, including the highly prevalent specific phobias. It is worth noting that the cognitive model firmly takes the view that anxiety and depression are continuous entities (Clark et al., 1999). It is thus appropriate to test the model with experiments that assume continuity.

Even if depression is truly a continuous condition, it is possible for studies labelled nonclinical and studies labelled clinical to arrive at discrepant results. There are several reasons for this. First, because differences between participants in studies of clinical depression are relatively large, and differences between participants in studies of subclinical depression are relatively small, at a given sample size clinical studies have more power to detect differences between groups. Second, subclinical and clinical populations differ in ways other than severity of depression; for example, clinical participants are more likely to have shown help-seeking behaviour and to be undergoing treatment, and they are less likely to have been selected from a student sample. The last difference means they are likely to be lower in IQ (Crawford \& Allan, 1997), which may affect some cognitive tasks. Moreover, is possible that some features of depression are continuous, and some are discontinuous (Flett et al., 1997). In particular, it remains possible that subclinical and clinical depression have discontinuous impacts on information processes. The review detailed below finds that clinical 
and nonclinical studies tend to find similar results in terms of information processing biases, although findings are sometimes weaker or less consistent in nonclinical studies. This is what the continuity view predicts. However, certain memory deficits appear to be associated with clinical, but not subclinical, depression, as predicted by a discontinuity view.

Although as argued by Coyne (1994), nonclinical studies do suffer from limitations, they also possess certain strengths that clinical studies do not. First, and most importantly for the current studies, nonclinical samples are useful for examining the relative effects of different components of negative mood. Clinically depressed individuals tend to be highly anxious, and clinically anxious individuals tend to be somewhat depressed (Clark \& Watson, 1991a). Within clinically depressed samples, level of depression and level of anxiety are very closely related (Enns, Cox, Parker, \& Guertin, 1998). The use of nonclinical samples allows experiments to more easily disentangle the differing information processing correlates of anxiety and depression, which is a main aim of this project. Similarly, the other main aim of this project is to compare the effects of the somatic and cognitive symptoms of depression. Clinically depressed individuals are likely to be very high on both types of symptoms, and so the use of nonclinical samples is a more powerful method for disentangling their effects. Second, nonclinical samples are easier to recruit, a practical advantage that can lead to the important methodological advantage of greater statistical power. Third, studies of information processing in clinical populations have difficulty controlling demand effects; participants presumably know that they have been selected into the study because they are anxious or depressed, and may feel that they are expected to behave in an anxious or depressed way. Studies using 
nonclinical populations can more easily minimise demand effects by concealing the purpose of the study for the duration of the experiment. Fourth, given that many depressed individuals do not seek help or undergo treatment, subclinical samples may be more representative of depressed people in general than are patients recruited from outpatient clinics (Vredenburg et al., 1993). Fifth and finally, the use of nonclinical samples may be less problematic for studies of information processing bias than for studies on certain other topics concerning depression or anxiety. Subclinically depressed students and clinically depressed outpatients have different demographic features (Coyne, 1994), and this is a problem for the investigation of contextual factors like daily stressors. However, demographics are of less concern to the study of basic cognitive processes, because cognitive psychology’s assumption of uniformity of cognitive architecture implies that the minds of people from different demographic groups will function in a similar manner (Coltheart, 2002).

These considerations suggest that it is important to conduct both clinical and nonclinical studies. For this reason, both clinical and nonclinical studies will be reviewed, but clearly labelled according to type of participant selection procedure used. The experiments in this thesis use nonclinical samples, because of their greater suitability for addressing the main aims of the project.

Statistical power. Cohen (1988) reviews evidence that insufficient power is a substantial methodological problem in research in social, experimental, and clinical psychology. An experiment's statistical power is the likelihood that it will find a significant effect in a sample, given that an effect of a certain size really does exist in the population from which the sample is randomly selected. 
Because effect sizes in many types of research are necessarily small, and participant numbers are often restricted by practical concerns, statistical power is often low. Cohen (1988) discusses meta-analyses that find that in many areas of psychology, the statistical power of the average published study to detect a small effect size is around 50\%. This means that even if a small effect size does exist, $50 \%$ of studies are likely to miss it, although publication bias may mean that not all of these studies will be published. This problem is likely to confuse the interpretation of research, and slow progress in an area, particularly when statistical power is not explicitly considered. At the extreme, Meehl (1990) argues that the combination of insufficient statistical power and the (partly consequential) submission and publication biases against studies with null results has rendered the results of whole fields of study uninterpretable. Unhappily, in the experimental cognitive abnormal psychology area, both limitations on effect size obtainable and limitations on the number of participants that can be recruited for a given experiment seem particularly intractable. Matt, Vazquez, and Campbell (1992) argue that the typical existing study has very low power to detect the small effect sizes that are being investigated. Before sufficient comparable studies have accumulated to allow meta-analysis, the best that can be done in this situation is to fully disclose the expected power of an experiment in the case of null results, and for power issues to be considered particularly carefully by narrative reviewers. At present, such considerations are rare; for example, in their review of the inconsistent findings in some areas of research in cognition and emotion, Williams et al. (1997) do not mention the issue of statistical power at all. Consideration of statistical power is also rare in the reporting of single experiments, though it may have recently been increasing in 
frequency. This thesis will discuss degree of statistical power in cases where null results are found.

\section{Mood and Memory}

Autobiographical memory. Autobiographical memory has long been thought to play a particularly important role in the emotional disorders (Williams, 1992). However, as several reviewers have noted, it is particularly difficult to study, because it is difficult to determine whether differences in valence of memories recalled are best accounted for by biased recall or by actual differences in life history (Blaney, 1986). Perhaps for this reason, there have been relatively few investigations examining naturally occurring mood and its relationship to valence of recalled events.

Mayo (1983) conducted a study of university students and staff. He found that valence of autobiographical memories produced in association to neutral cue words was not significantly related to despondency, anxiety, or happiness, as measured by simple visual analogue scales, but was related to personality, as measured by the Eysenck Personality Questionnaire (EPQ). However, this study was not a fair comparison of the effects of mood and personality, as the EPQ is likely to be a more valid and reliable measure of personality than visual analogue scales are of current mood. Also, the analogue scales used in this experiment rate immediate mood, while depression and anxiety inventories rate mood over the preceding one or two weeks. Mayo (1989) examined a similar population and found similar but weaker effects of personality, and not all of his earlier findings were replicated in this study. Anxiety was also measured using the STAI-Trait, 
and was associated with the production of unhappier memories. Immediate mood state was also measured, but correlations with valence of recalled memories were not reported.

In addition to looking at the emotional valence of freely recalled memories, researchers have looked at the time taken to recall either happy or unhappy memories. The results have sometimes but not always been supportive of the mood-congruent hypothesis. Williams and Scott (1988) found that depressed individuals took longer to produce happy memories than did nondepressed individuals. Richards and Whittaker (1990) also found a moodcongruent effect: self-reported depression was related to response latency for negative memories, and self-reported anxiety was related to response latency for positive memories. Similarly, MacLeod, Andersen, and Davies (1994) found that self-ratings of positive and negative affectivity predicted recall latency for autobiographical memories cued by the terms used in the positive and negative affectivity schedule (PANAS; Watson, Clark, \& Tellegen, 1988). However, Kuyken and Dalgleish (1995) found that depressed individuals were no different from nondepressed individuals in their pattern of response latencies for positive and negative events.

As already noted, it is uncertain whether these mood-congruent results are due to biased cognitive processes or to veridical differences in participants' life histories. One way of avoiding the confounding of biases in recall with veridical differences in events is to capitalise on natural variations in mood. Fogarty and Hemsley (1983) found that valence of recalled memories changes in a manner congruent with recovery from clinical depression. Similarly, Hammen, Miklowitz, and Dyck (1986) asked college students to provide examples of their 
past behaviours that were consistent with positive or negative words that they had judged to be self-descriptive. Subclinically depressed participants provided fewer positive examples, and many more negative examples, than nondepressed participants. Participants who had recently been subclinically depressed performed identically to the stably nondepressed participants. Thus, both studies seem to show that current mood state, rather than veridical differences in life history, is responsible for the recall of more negative events in depression. However, Blaney (1986) argued that because in studies like the ones described above changes in mood occur over a long time frame, the findings of studies with this design could be related to a change in the pleasantness of the events that participants had recently experienced, rather than to a mood-related change in recall bias. Clark and Teasdale (1982) avoided this problem by investigating valence of autobiographical events recalled at different times of the day. The participants were clinically depressed individuals with strong diurnal variation in depression, and they recalled autobiographical events that varied in valence in a manner that was congruent with current mood. Consistent with the studies already discussed, this study suggests that depression is associated with a negative bias in autobiographical recall.

A second way of avoiding the confounding of biases in recall and actual differences in events is to artificially manipulate mood. Teasdale and Fogarty (1979) found that when participants were inducted into depressed or elated moods using a variant of the Velten self-statement procedure, their recall latencies for pleasant and unpleasant memories were affected in a moodcongruent way. Teasdale and Russell (1983) conducted a similar study without constraining participants to produce either pleasant or unpleasant memories. 
Instead, participants responded to neutral cue words by freely recalling memories of any emotional valence. The authors replicated their earlier response latency finding, and also found that negative mood induction was associated with the production of less pleasant autobiographical memories. However, Ehrlichman and Halpern (1988) argued that the effects produced in these studies could have been produced by non-emotional cognitive mechanisms related to the semantic content of the self-statements used to induce the mood. To avoid this problem, these authors used pleasant or unpleasant odours to induce positive or negative mood, and found that individuals in the pleasant odour condition recalled happier memories than those in the unpleasant odour condition. More recently, Kumari, Hemsley, Cotter, Checkley, and Gray (1998) investigated the effects of a placebo and of the mood-lowering drug haloperidol on recall of personal memories. They found that the drug caused participants to recall unhappier memories. It could, however, be argued that the effect of the drug on recall was directly biologically mediated, rather than caused by mood itself, an idea consistent with the fact that the change in valence of recalled events was only partially mediated by self-rated mood. Despite some individual methodological weaknesses, in combination these consistent strands of evidence suggest that induced mood-congruent autobiographical recall is a robust and genuine effect.

Clinical observation has suggested that depressed individuals are not only more negative in the memories that they recall, but that they are also less able to recall certain kinds of specific events (Williams, 1992). This lack of specificity has also been the focus of research attention. Overgeneral recall is the inability to remember specific events from one's own life - that is, events that occurred only once and were less than one day in duration (Williams, 1996). Early research 
found that overgeneral memory was found in suicide attempters (Williams \& Broadbent, 1986), but more recent investigations have focused on the relationship between depression and overgeneral memory. Kuyken and Dalgleish (1995), Moore, Watts, and Williams (1988), and Williams and Scott (1988) have all found that clinically depressed individuals produce more overgeneral memories than do controls; this effect seems to be quite consistent. Wessel, Meeren, Peeters, Arntz, and Merckelbach (2001) found that overgeneral memory was specific to major depressive disorder, and was not related to the presence of an anxiety disorder or to self-rated depression severity. However, Wessel et al. (2001) did not examine any participants diagnosed with post-traumatic stress disorder, and other studies have found that this specific anxiety disorder is associated with overgeneral memory (McNally, Lasko, Macklin, \& Pitman, 1995). Consistent with Wessel et al.'s (2001) finding that self-rated depression was unrelated to overgeneral recall, Merckelbach, Muris, and Horselenberg (1996) investigated a large, unselected student sample, and found that overgeneral memory was not associated with trait anxiety, neuroticism, or depression, all as measured using self-report inventories. Williams, Williams, and Ghadiali (1998) have obtained similar findings, although it is doubtful that their sample size of 15 would provide sufficient power to detect mood related differences. Merckelbach et al. (1996) did find that overgeneral memory was associated with left-hemispheric thinking style as measured by a paper-andpencil questionnaire. The influence of thinking style on information processing is discussed in more detail later in this introduction.

Merckelbach et al.'s (1996) finding that depression, as measured by selfreport inventory, is unrelated to overgeneral memory is more puzzling than it 
might seem. These authors argued that this finding means that overgeneral memory is a stable trait that predicts vulnerability to clinical depression, rather than an unstable product of transient mood disturbance. Yet persons scoring highly on self-report depression inventories are more likely than persons scoring low on these inventories to report past or present clinical depression, and are also more susceptible to developing clinical depression in the future (Gotlib et al., 1995). Thus even if we assume that only clinical depression as diagnosed with a structured interview produces overgeneral memory, many of those who score highly on depression inventories fit this criterion, and this should increase the mean level of overgeneral recall found in this group. There are three possible reasons that this might not occur. First, there could be a subgroup of high scorers who are lower in overgeneral recall than low scorers, to balance out the overgenerality of the clinically depressed individuals (Dawes, 2001). There is no obvious theoretical reason for this to occur. Second, a relationship might in fact exist. In this case, the null results of the one experiment that has examined overgeneral memory in nonclinical depression would have been due to chance; this is possible as Merckelbach et al. (1996) did not report the power of their study. Third, findings of overgeneral memory in clinically depressed individuals could be due to some methodological artefact; for example, the use of antidepressants in the clinical groups, which existing experiments have made only indirect efforts to control. Experiment 2 of this thesis seeks to further confirm Merckelbach et al.'s (1996) finding that overgeneral memory is unrelated to nonclinical depression, and will report the statistical power of the comparison. Studies examining whether overgeneral memory plays a casual role in depression have produced mixed results. Mackinger, Pachinger, Leibetseder, and 
Fartacek (2000) found that women who had been previously depressed were more overgeneral in their memories than women who had not been previously depressed. However, Wessel et al. (2001) found that previous depression was not related to overgeneral recall. Similarly, some authors but not others have found evidence for predictive validity of overgenerality in autobiographical memory. Brittlebank, Scott, Williams, and Ferrier (1993) found that overgeneral recall predicted poor response to antidepressant treatment. Brewin, Reynolds, and Tata's (1999) study did not replicate this finding, but it did find that overgeneral memory was associated with the presence of spontaneous intrusions of distressing memories, and that this latter variable predicted poor treatment response. More consistently, overgeneral recall has been found to be associated with poor social problem solving (Goddard, Dritschel, \& Burton, 1996; Sidley, Whitaker, Calam, \& Wells, 1997), although the authors of these studies did not control for the possible mediating effect of IQ. This is an important flaw, because the ability to produce specific autobiographical memories is strongly related to IQ (Williams et al., 1998), and social problem solving is also likely to be related to IQ, because performance on most complex intellectual tasks is associated with this variable (Jensen, 1998). Although evidence for the causal role of overgeneral memory in depression could be seen as weak, some attention has already been paid to the possibility of using interventions to reduce overgeneral recall (Watkins, Teasdale, \& Williams, 2000; Williams, Teasdale, Segal, \& Soulsby, 2000).

In summary, studies of natural variation in mood have found that autobiographical memory is mood-congruent. Mood manipulation studies have demonstrated that part of this effect is due to differences in cognitive processes 
rather than differences in veridical life history. At this stage, there is insufficient evidence to determine whether depression, anxiety, or both are driving the effect. In contrast, overgeneral recall characterises major depression, suicidality, and post-traumatic stress disorder, but is not characteristic of the other anxiety disorders or of nonclinical disturbed mood states.

Nonautobiographical memory. Studies of material learnt in the laboratory have the advantage that there is precise control over the stimuli to which the participants are exposed. They also have the disadvantage that the material to be recalled is less personally relevant. Some authors have hypothesised that this aspect of the word learning tasks may weaken any effects found (Blaney, 1986).

Mood congruence in nonautobiographical memory has been extensively investigated. For this reason, the literature will not be reviewed in detail: instead, the conclusions of various existing reviews will be discussed, as will a selection of the most recent studies. The majority of studies have investigated explicit memory, that is, participants were explicitly asked to recall a previously presented word set.

Blaney (1986) conducted the earliest significant review of studies of mood and memory. Anxiety was not addressed by the review. Blaney (1986) concluded that there was evidence for mood-congruent memory in induced mood, naturally occurring nonclinical depression, and clinical depression. He also concluded that mood-congruence does not appear when self-referent learning of the materials is explicitly discouraged during the learning phase (for example, when participants are asked to count the number of times the letter $\mathrm{E}$ appears in each word). More recently, Matt et al. (1992) conducted a metaanalytic review of published studies of mood-congruent recall. Again, anxiety 
was not discussed. For naturally occurring mood, they found evidence that nondepressed individuals showed a positive bias in recall, dysphoric individuals showed even-handed recall, and clinically depressed individuals showed a negative bias in recall. Statistical tests generally suggested homogeneity of effect size - that is, there was no evidence that methodological differences between studies had any effect on the results obtained, except for in the case of induced elated mood. However, consistent with Blaney (1986), Matt et al. (1992) noted that there was a trend for non-self-referent encoding of information to be less likely to yield mood-congruent recall.

Matt et al. (1992) also make two important methodological points. First, the mean effect sizes found were small to very small according to Cohen's (1988) effect size conventions. Matt et al. (1992) calculate that most studies of moodcongruent recall are too small to have sufficient power to detect the effects of interest; for example, if the effect of clinical depression on mood-congruent recall is of the average size calculated in the review, a typical study of clinical depression has only a 13\% chance of correctly finding that clinically depressed individuals are negatively biased in their recall. Second, Matt et al. (1992) conducted analyses to determine how many unpublished studies with null results would need to exist for the average effect size found by a review that also included these studies to be nonsignificant; in all cases but that of the positive bias in nondepressed participants, this number was small, and so Matt et al. (1992) suggest caution in interpreting their meta-analytic findings.

There is one final notable finding of this review. As discussed in the section on methodology, individuals classified as nondepressed in research studies often score appreciably lower on depression inventory score than the 
average of the population. Matt et al. (1992) found that "nondepressed" individuals actually showed a stronger positive bias than unselected, induced elated, individuals. This suggests that the positive bias shown by the control groups in these studies is the result of the selection process used, and is not necessarily characteristic of the general population.

A significant number of studies examining mood-congruent memory have been published since Matt et al. (1992) conducted their meta-analytic review, but subsequent reviews have been narrative rather than meta-analytic. These reviews have consistently argued that both nonclinical and clinical depression produce mood-congruent recall, but that anxiety rarely does so (Dalgleish \& Cox, 2000; Ellis \& Moore, 1999; Gotlib \& Krasnoperova, 1998; Gotlib \& Neubauer, 2000; MacLeod \& Rutherford, 1998; Williams et al., 1997). The results of empirical studies subsequent to Matt et al.'s (1992) review continue to be mixed; for example Denny and Hunt (1992) found that clinically depressed individuals were more negative in their recall than controls, but Banos, Medina, and Pascual (2001) did not. Similarly, Watkins, Mathews, Williamson, and Fuller (1992) found that subclinically depressed individuals were more negative in their recall than controls, but Lang and Craske (1997) did not.

Finally, investigators have also examined mood-congruent biases in implicit memory. Implicit memory for word lists occurs when responses are affected by previously presented information without the participants being aware of this. Implicit memory is often assessed using either word stem or word fragment completion tasks. For example, Mathews, Mogg, May, and Eysenck (1989) asked participants to imagine themselves in scenes related to a number of positive, negative, and neutral words. They then completed a nonsemantic 
distracter task, and then a word-stem completion task. The word-stem completion task involved thinking of words to complete three letter stems (for example, FAI___ ); for some of the word stems, one possible answer was a word the participant had been previously exposed to in the imagination task. Participants were more likely complete a word stem with a given word if they had been previously exposed to it, thus demonstrating implicit memory. More importantly, the clinically anxious participants showed relatively more implicit memory for the negative material than did the control participants. This demonstrated a mood-congruent implicit memory bias. Studies have found that both clinical and nonclinical levels of anxiety are related to mood-congruent implicit memory (Eysenck \& Byrne, 1994; Lang \& Craske, 1997; Mathews, Mogg et al., 1989). However, Banos et al. (2001) did not find an implicit memory bias in a group of panic disordered patients. In contrast to the overall findings for anxiety, several studies have found that neither clinical nor nonclinical depression are associated with a mood-congruent bias in implicit memory (Banos et al., 2001; Danion, Kauffmann-Muller, Grange, Zimmerman, \& Greth, 1995; Denny \& Hunt, 1992; Lang \& Craske, 1997; Watkins et al., 1992). Roediger and McDermott (1992) suggested that depression might be associated with mood-congruent bias in conceptual, but not perceptual, implicit memory. They made this suggestion because it had been hypothesised that depression affects elaboration, rather than perceptual priming (Williams, Watts, MacLeod, \& Mathews, 1988; see also the section on theory in this thesis). Watkins, Vache, Verney, and Mathews (1996) tested the conceptual-perceptual hypothesis by conducting a conceptually-based implicit memory task with clinically depressed university students. Their encoding task was an imagination task, their recall task was a free-association 
task, and they found mood-congruent implicit memory. Watkins, Martin, and Stern (2000) conducted a similar study involving two perceptual implicit memory tasks and two conceptual implicit memory tasks - the free association task used in their 1996 study, and a word-retrieval task that required participants to produce words to fit supplied definitions. Participants showed mood-congruent recall on the word-retrieval task, but not on the other three tasks. The authors concluded that depression-congruent implicit memory occurs only on conceptual tasks, but that even then the effect is dependent on the exact experimental method used. Somewhat inconsistent with this conclusion and the other existing evidence, Ruiz-Caballero and Gonzalez (1994) did find a depression-congruent perceptual implicit memory bias in subclinically depressed participants using a word-stem completion task.

Some investigators have taken a less conventional approach to the measurement of implicit memory, by using a subliminally primed lexical decision task (Bradley, Mogg, \& Millar, 1996; Bradley, Mogg, \& Williams, 1994, 1995; Scott, Mogg, \& Bradley, 2001). In these experiments, the target stimuli are strings of letters, and the participants' task is to decide whether or not these stimuli form valid words. Some stimuli are preceded by subliminally presented prime words. When participants classify primed stimuli as words more rapidly than unprimed stimuli, priming has occurred. When mood-congruent stimuli are primed more than mood-incongruent stimuli, mood-congruent bias has occurred. In these experiments, the prime word could either be the same as the target word (repetition priming) or a closely related word (semantic priming). The repetition priming experiments used upper case letters as primes, but lower case letters as target words, in an effort to ensure that any effect was due to 
conceptual, rather than perceptual, processes. However, many letters do share perceptual properties between their upper and lower case forms, so classification of this task as conceptual or perceptual is ambiguous (Scott et al., 2001). Bradley et al. (1994, 1995, 1996) found that both clinical and nonclinical participants showed mood-congruent biases on the repetition priming task. Scott et al. (2001) found that nonclinical participants did not show mood-congruent biases on the repetition priming task, but did show mood-congruent biases on the semantic priming task. Across the series of experiments, mood-congruent bias was more strongly and consistently associated with depression than with anxiety. Overall, the findings are consistent with the view that depression is associated with conceptual implicit memory bias (Scott et al., 2001). However, one limitation of the subliminal priming paradigm is that the effects appear to occur over a limited duration; no mood-congruency effects were found after a delay of two seconds (Scott et al., 2001). Thus, it is unclear that these mood-congruency effects are in fact due to memory processes. Bradley et al. $(1994,1995,1996)$ did also include a supraliminal priming condition that had a longer delay between presentation of prime and target stimuli. Consistent with their other findings, they found depression-congruent processing, but as previously noted it is unclear whether these experiments were measuring perceptual or conceptual implicit memory. In summary, depression appears to sometimes produce a bias in conceptual implicit memory, but to less often produce a bias in perceptual implicit memory. Anxiety appears to produce a bias in perceptual implicit memory, but the effects of anxiety on conceptual implicit memory have not been sufficiently investigated. Because anxiety and depression co-vary, but research in this area has typically investigated only one of these variables, it is hard to 
determine whether the effects demonstrated by individual studies are due to anxiety or to depression. The need for a study that concurrently investigates both anxiety and depression, and both conceptual and perceptual implicit memory, is apparent. Experiment 1 of this thesis was designed to address this need.

Studies have also examined whether emotional state affects ability to explicitly recall neutral materials. For measures of explicit memory, the pattern of findings is similar to that already described for overgeneral recall in autobiographical memory. Burt, Zembar, and Niederehe (1995) conducted a meta-analysis that found that clinical depression was associated with an explicit memory deficit that was small to moderate in Cohen's (1988) effect size terms. The effect was larger in inpatient than outpatient samples. The available evidence suggests that no explicit memory deficit is associated with either subclinical depression (Gotlib, Roberts, \& Gilboa, 1996) or anxiety disorders that are not accompanied by comorbid depression (Burt et al., 1995). The similarity of the mood states that are associated with impairment of explicit wordlist recall and with impairment of the ability to produce specific autobiographical memories suggests a need to investigate whether a common mechanism causes both impairments, but existing research does not address this question. Experiment 2 contains a preliminary investigation of this matter.

Finally, studies have examined whether depression is related to deficits in implicit memory. Clinical depression does not seem to be associated with deficits in perceptual implicit memory (Burt et al., 1995; Jenkins \& McDowall, 2001), but recent evidence suggests it may be associated with deficits in conceptual implicit memory (Jenkins \& McDowall, 2001). There is no corresponding 
evidence that a similar deficit in conceptual implicit memory occurs in subclinical depression.

In summary, depression seems to be closely related to explicit and conceptual implicit memory biases and deficits, and anxiety seems to be closely related to perceptual implicit memory biases. However, these distinctions are not always clear cut and consistent. The picture is blurred by methodological and population differences between studies, the difficulty of attracting enough participants to allow sufficient power to detect the effects of interest, and the failure of many authors to measure both anxiety and depression within the same study.

\section{Mood and Attention}

Studies of attentional processing in anxiety and depression have adopted two main approaches. The first approach has been to measure interference effects. Interference effects occur when participants perform worse on a nonemotional task when emotional stimuli are used. The experimenters who use interference tasks assume that the interference effects occur when the emotional stimuli capture the attentional resources of the participants. The second approach to examining attention in depression and anxiety has been to measure facilitation effects. Facilitation effects occur when participants perform better on a nonemotional task when task stimuli and emotional stimuli are presented in the same spatial region. The experimenters who conduct this research assume that facilitation effects occur when the participant's attention is automatically oriented towards the emotional stimuli. 
Attentional interference has usually been measured using the emotional Stroop task. In the emotional Stroop task, participants name the colours of neutral or emotionally valenced words. The theory behind the task is that emotionally disturbed individuals will be more distracted by negative stimuli, and will thus be slower to name the colour of these stimuli. There has been a large number of studies utilising the emotional Stroop task, and only a representative sample is reviewed here. Many studies have found that anxious participants have shown an emotional Stroop interference effect (Williams et al., 1997), so the review will focus on the more controversial issue of whether there is an emotional Stroop effect in depression. Gotlib and Krasnoperova (1998) and Horowitz et al. (1997) argue that depression is characterised by increased Stroop interference for negative stimuli. Williams, Mathews, and MacLeod (1996) also appear to support this position. Two early experiments produced results consistent with this conclusion - Gotlib and McCann (1984) and Gotlib and Cane (1987) found that depression was associated with greater colour naming interference of depression-related words in a nonclinical student sample and in a clinical sample, respectively. Similarly, Klieger and Cordner (1990) found an emotional Stroop effect for mildly dysphoric students, though they found no effect for moderately dysphoric students. However, none of these experimenters measured anxiety in their participants. This leaves open the possibility that the depressed groups in these experiments were characterised by higher levels of anxiety than the control groups, and that it was this greater level of anxiety that produced the emotional Stroop effect. Depression, in itself, could have had no effect at all. Studies that have examined both anxiety and depression lend support to this possibility. Mathews and MacLeod (1985) ran a Stroop task on controls 
and on outpatients who had been referred by general practitioners for anxiety management training. These authors measured state and trait anxiety using the STAI, and depression using the Beck Depression Inventory (BDI). Looking at the sample as a whole, STAI-Trait, STAI-State, and BDI scores were all significantly correlated with emotional Stroop interference. However, when partial correlations were calculated for these variables (in each case, controlling for the other two measures used), only state anxiety remained significantly related to the interference effect. Thus, it is plausible that the effect of depression on Stroop interference was mediated by state anxiety. Dalgleish (1995) chose 12 high and 12 low anxious students based on their STAI-Trait scores. He conducted an emotional Stroop task using button press responses to categorized neutral, positive and threat words. As well as calculating group differences, he calculated correlations between measures of emotional disturbance and degree of threat word interference, although these correlations may have been inflated by the highly selected nature of the sample. Threat word interference was most highly related to STAI-State score $(\underline{r}=.67)$ but it was also related to STAI-Trait $(\underline{r}=.56)$ and BDI scores $(\underline{r}=0.44)$. Partial correlations for each of the mood measures with Stroop interference, controlling for the other mood measures, were nonsignificant. Given the small sample size used in this experiment, and the likelihood that there were very high intercorrelations between the mood measures, this is unsurprising.

MacLeod and Hagan (1992) ran the emotional Stroop on a nonclinical, nonstudent sample, unselected for depression or anxiety, and found that when emotional threat words were presented supraliminally, there was no association between threat word interference and self-reports of either anxiety or depression. 
When the threat words were presented subliminally, both the STAI-Trait and STAI-State were significantly related to interference. There was a nonsignificant trend for the BDI to relate to interference; again, if this trend represents a real effect, it may have been caused by the relationship of depression to anxiety. Finally, Hill and Knowles (1991) found no effect of subclinical depression on emotional Stroop interference. In fairness to the case for an effect of depression on the emotional Stroop, it should be noted that their small sample size (24 participants in all) is likely to have resulted in very low power. However, the weight of the evidence from these subclinical studies strongly suggests that interference on the emotional Stroop is due to anxiety, not depression.

Studies of clinically anxious and depressed individuals have tended to confirm that Stroop interference is more closely related to anxiety than to depression. McNeil, Tucker, Miranda, Lewin, and Nordgren (1999) investigated performance on two emotional Stroop tasks, one of which used depression related stimuli and the other of which used anxiety related stimuli. Participants were clinically diagnosed with obsessive-compulsive disorder, post-traumatic stress disorder, or major depressive disorder. Overall, the participants were slower when responding to emotional words, but when the groups were analyzed individually, this effect was significant only in the post-traumatic stress disorder group. There was no evidence that matching type of negative stimuli to participant diagnosis produced more interference than nonspecific negative stimuli. Carter, Maddock, and Magliozzi (1992) also ran Stroop experiments with depression and anxiety related stimuli, this time on controls and on participants with either panic disorder or clinical depression. Panic disordered participants showed significant interference for both kinds of stimuli, while 
clinically depressed participants showed a non-significant trend towards showing interference for depression-related stimuli only ( $\mathrm{p}=0.11)$, despite reporting higher levels of trait anxiety than the panic disordered participants. Bradley, Mogg, Millar, and White (1995) obtained findings even more inconsistent with the idea that depression can produce an emotional Stroop effect - they found that participants with an anxiety disorder alone, but not control participants or participants with a comorbid anxiety and depression diagnosis, showed an interference effect for negative words. That is, the presence of severe depression appeared to actively suppress the emotional Stroop effect.

In summary, some studies have found an association between depression and slowed colour naming of emotional words. However, these studies have failed to rule out the plausible hypothesis that anxiety mediates this difference. Because of the known association between depression and anxiety, no study can convincingly demonstrate a potentially causal link between depression and emotional Stroop interference unless it also measures the association between concurrent anxiety and Stroop interference. No study that has done this has found a significant association between current depression and emotional interference that could not plausibly be mediated by anxiety. Finally, although many studies have found an emotional Stroop effect related to anxiety, the effect has not been entirely consistently replicated, even at clinical levels of distress (Dozois \& Dobson, 2001; Kyrios \& Iob, 1998).

Although the emotional Stroop task has been a very popular research tool, several authors have argued that the emotional Stroop effect cannot be unambiguously interpreted as demonstrating an attentional bias (Gotlib \& MacLeod, 1997). In the conventional Stroop task, it is unclear that the 
interference effect can be strictly attributed to attentional processes (MacLeod, 1991), and this problem also applies to the emotional Stroop (Williams et al., 1996). Similarly, De Ruiter and Brosschot (1994) argue that the emotional Stroop effect may be due to anxious participants utilising cognitive avoidance, rather than selectively attending to the negative information. Tasks examining facilitation effects have examined the influence of mood on attention more directly.

The first facilitation task used was the dot-probe task. The dot-probe task is a reaction time task, in which dots are presented somewhere on a computer screen. The participant responds with a key press as rapidly as possible. Neutral or emotional words are presented in different regions of the computer screen. Allocation of attention is demonstrated by facilitated reaction time - that is, if participants respond more rapidly to dots presented near the negative stimuli, then it is assumed that their attention is being allocated to the negative stimuli.

Reviewers have concluded that anxious participants show consistent negative biases in attention on the dot probe task (Gotlib \& MacLeod, 1997). However, similarly to studies utilising the emotional Stroop, studies using the dot probe task have found inconsistent relationships between depression and deployment of attention. MacLeod, Mathews, and Tata (1986) found that clinically anxious participants showed biases towards negative information, but clinically depressed participants did not. However, controls showed a bias away from negative information, so the attentional deployment of the clinically depressed individuals cannot be considered normal. Similarly, Hill and Dutton (1989) found no difference between nonclinically depressed individuals and controls on a dot-probe task. Three more recent studies have found a negative 
attentional bias on the dot probe task for both clinically (Mathews, Ridgeway, \& Williamson, 1996; Mogg, Bradley, \& Williams, 1995) and nonclinically depressed populations (Westra \& Kuiper, 1997). Gotlib and Neubauer (2000) suggest that these positive findings are due to a methodological refinement in these studies, although it is unclear why this methodological refinement should be necessary to find an effect for depression, but not for anxiety.

A second kind of facilitation task is the Deployment of Attention Task (DOAT) developed by Gotlib, McLachlan, and Katz (1988). In the DOAT, participants are asked to fixate on a central crosshair, and are then presented with two words, one above and one below the crosshair. The words are then replaced with coloured bars. The participants are asked to determine which bar appeared first; in fact, the bars appear simultaneously. Prior research has demonstrated that people perceive events as occurring sooner when they have allocated their attention to that spatial area. Therefore, when participants perceive bars that replace words of a certain valence as appearing sooner, it is inferred that they are preferentially allocating their attention towards this word valence.

Gotlib et al. (1988) compared the performance of subclinically depressed participants on the DOAT to the performance of controls and, unexpectedly, found that controls showed a bias away from negative stimuli, but subclinically depressed participants did not. Mogg et al. (1991) compared subclinically anxious participants and controls, and clinically anxious participants and controls, and found no evidence of bias in any group. However, they used a shorter stimulus exposure time in these experiments than had been used in Gotlib et al.'s earlier experiment (500 ms vs. 730 ms). The same authors then compared high and low anxious students, using a median split on STAI-State score and a 
$730 \mathrm{~ms}$ presentation time. They found that low-anxious participants were positively biased, but high-anxious participants were not. McCabe and Gotlib (1995) compared clinically depressed participants to controls and found that controls were positively biased but that clinically depressed individuals were not. Finally, McCabe and Toman (2000) compared subclinically depressed participants to controls and found that controls were positively biased but subclinically depressed individuals were not. This pattern of results was found regardless of stimulus presentation duration $(750-1500 \mathrm{~ms})$. The authors of this series of studies concluded that clinically and subclinically depressed participants, and subclinically anxious participants, are even-handed in their attention, but that "normal" controls show a positive bias.

This series of studies is interesting in that each study has sought to measure both anxiety and depression using self-report measures. The three most recent studies have also measured both state and trait anxiety. However, three of the four studies suffer from a methodological problem in differentiating anxiety from depression. Gotlib et al. (1988), Mogg et al. (1991), and McCabe and Gotlib (1995) used the STAI to measure anxiety, and this instrument differentiates anxiety from depression poorly (Bieling, Antony, \& Swinson, 1998; Endler, Cox, Parker, \& Bagby, 1992). Perhaps as a result, the studies have been inconsistent in their findings as to whether depression or anxiety is more closely related to attentional bias - Gotlib et al. (1988) found anxiety and depression to be equally important, Mogg et al. (1991) found anxiety to be more important than depression, and McCabe and Gotlib (1995) and McCabe and Toman (2000) found depression to be more important than anxiety. Experiment 1 
of this thesis addressed this issue by using a measure of anxiety that has been found to be relatively separate from depression.

This series of studies also illustrates a point made in the section on studies of clinical and subclinical depression. In each of these studies, the control group was significantly lower in self-reported distress than is the general population (Beuke, 2002). Thus, the positive biases shown by the control groups of these studies may have been present because these groups were unusually happy or calm. Experiment 1 of this thesis addresses this issue by using an unselected sample.

In summary, studies of attentional processing have found that interference effects are fairly consistently shown by anxious participants, but are less consistently shown by depressed participants. Facilitation effects occur consistently in anxiety, and also occur consistently in depression on the DOAT task, but less consistently on the dot probe task.

\section{Mood and Judgement}

Like studies of mood-congruent memory, studies of mood-congruent judgement have taken two main approaches: inducing mood in normal participants, and investigating participants with naturally elevated anxiety or depression. Using the first strategy, Forgas, Bower, and Krantz (1984) hypnotically induced participants into happy or unhappy moods, and had them rate behaviour shown during an interview videotaped in a previous session. Participants rated both their own and another person's behaviour more negatively when induced into the unhappy, rather than happy, mood, and this effect was 
particularly pronounced for self-ratings. This study was limited by the fact that it did not include a neutral condition; so it is possible that the happy participants were the ones who showed mood-congruent biases, and that the unhappy participants were unbiased. The use of hypnotism may also have created demand effects. Johnson and Tversky (1983) found that using a newspaper article referring to a tragic event to induce negative mood raised subjective estimates of the likelihood of unrelated risks. Schwarz and Clore (1983) asked participants to judge their satisfaction with their own lives. They induced positive or negative mood either by asking participants to recall a positive or negative event, or by interviewing participants on either a sunny or a rainy day. Mood induced by either method affected judgement of life satisfaction. The method of using natural weather variation to induce mood is particularly impressive, in that the resulting effect on judgement seems unlikely to have been caused by nonemotional associative processes of the kind that could operate in studies using Velten inductions, or by demand effects of the kind that could operate in studies using hypnotism.

Studies of naturally occurring anxiety and depression have also found evidence of mood-congruent judgement. Krantz and Hammen (1979) found that both clinically depressed participants and participants scoring higher than average on the BDI were more likely than controls to select distorted negative interpretations of hypothetical events. Kuiper and MacDonald (1983) found that estimates of the frequency of positive and negative events in the general population, and of the likelihood of depressed or elated responses to those events, were congruent with mood as measured with the BDI. In another study, Wenzlaff and Bates (1998) asked subclinically depressed or nondepressed participants to 
unscramble sentences. The participants could unscramble the sentences in either a positive or a negative way. Mood-congruent interpretation of the sentences was found.

Each of these studies has measured or manipulated only one of the three relevant components of negative emotion: depression, anxiety, or general negative affect. This causes the problem of interpretation discussed in the section on anxiety and depression. Because anxiety and depression are closely related, and participants selected on one of these variables are also likely to be high on the other, none of these studies elucidate whether negative biasing of judgement is related to anxiety, depression, or both. One early study that did measure both anxiety (using the STAI-Trait) and depression (using the BDI) was conducted by Zarantonello, Slaymaker, Johnson, and Petzel (1984). These authors had participants perform an anagram unscrambling task. The variable of interest here is the participants' subjective evaluation of their own performance. Both the high-depression and the high-anxiety groups gave self-ratings of their own performance that were more negative than those of controls, and this effect was not solely due to real differences in performance. Subsequent analyses suggested that the relationship between depression and subjective rating of performance was mediated by anxiety; that is, depression itself had no direct effect on selfevaluation. This finding suggests that anxiety may be a more important influence on judgement tasks than depression, but the evidence for this conclusion is weakened by the use in this study of the STAI-T, which as discussed elsewhere does not discriminate well between depression and anxiety.

All of these studies used procedures that clearly made their participants aware that they were being given a choice between making either a negative or a 
positive response. This means that the results of these studies could plausibly be explained in terms of demand effects (MacLeod, 1990), especially in those studies where the selection process or mood induction procedure is clear to the participants. Eysenck, MacLeod, and Mathews (1987) conducted the first experiment using a now popular methodology that evaluates how participants interpret ambiguous information when it is not made clear that disambiguation is the focus of study. This methodology is the homophone interpretation task, and in it participants are aurally presented with a word list, and asked to write the words down. The task is usually falsely presented to the participants as being a word comprehension or spelling task. Some of the words presented are neutral and unambiguous, or neutral/neutral homophones, but some are negative/neutral or positive/neutral homophones, for example "bored/board” or "presents/presence”. It is assumed that the participants record the words in the way that they naturally interpret them. Mood-congruent results on this task have been found for anxiety as measured using the STAI (Byrne \& Eysenck, 1993; Eysenck et al., 1987; Mogg et al., 1994), in clinical anxiety states (Mathews, Richards, \& Eysenck, 1989), in subclinical depression (Wenzlaff \& Eisenberg, 2001), and in induced and naturally occurring negative affective states (Halberstadt, Niedenthal, \& Kushner, 1995; Rusting, 1999). None of these studies have shown whether depression, anxiety, or both are responsible for the negative interpretative bias shown in these studies, because none of these studies has attempted to directly compare these variables. Experiment 2 of this thesis included such a direct comparison.

Somewhat ironically, all of the studies that have just been described have possessed an interpretative ambiguity in their implications, as well as in their 
stimuli. MacLeod and Mathews (1991) argue that the results found in these studies could arise from either of two phenomena. First, anxious and depressed individuals could be showing a genuine negative interpretative bias. Second, anxious and depressed individuals could be identical to controls in their interpretation of ambiguous stimuli, but be more likely than controls to emit negative responses. The latter possibility would mean that the results of the studies reviewed were due to a response bias rather than to an interpretative bias. MacLeod (1990) investigated this possibility. In this study, participants were selected for either high or low trait anxiety. In the experimental session, participants read aloud sentences, and then read aloud a target word as quickly as they could. Target words were either semantically related to the sentence presented (primed condition), or semantically unrelated to the sentence presented (unprimed condition). Under normal circumstances, primed target words are read more quickly than unprimed target words. The critical condition occurred when sentences that could be interpreted in either a neutral or negative way were presented. These critical sentences were followed by target words related either to the neutral or to the negative interpretation of the sentence. The participants' interpretations of the ambiguous sentences were inferred by their pattern of priming - if the neutral words showed greater priming, it was inferred that the participants were making neutral interpretations, but if the negative words showed greater priming, it was inferred that the participants were making negative interpretations. MacLeod (1990) found that high trait-anxious participants who were also high in state anxiety showed greater priming for the negative words than did the other participants, suggesting that they made more negative interpretations of the ambiguous sentences. This result suggests that this 
subgroup of anxious individuals genuinely interpret ambiguous stimuli more negatively, rather than solely showing a negative response bias.

Lawson and MacLeod (1998) conducted a similar study, this time examining depression. After a large pre-test session, they selected participants who were in the top or bottom third of BDI scores, but in the middle third of STAI-Trait scores. The study found that for the low BDI group, reaction time to words related to the negative meaning of the sentence was facilitated, but reaction time to words related to the neutral meaning of the sentence was not. In contrast, for the high BDI group, reaction times to words related to the neutral meaning of the sentences were facilitated, but reaction time to target words related to the negative meanings of the sentences were not. This finding suggests low BDI participants were more negatively biased than the high BDI participants were - exactly the opposite finding to that predicted. The authors expressed some surprise before accepting their results.

If, however, one assumes that an interpretive bias is due to anxiety and not to depression, then the findings of Lawson and MacLeod's study can be explained with reference to specific design features of the study and to the tripartite model of negative emotion (Clark \& Watson, 1991b). Recall that the high and low BDI groups of this study were matched on STAI-Trait score, a manipulation that required inviting a particularly select group of participants to take part in the study. The tripartite model of negative emotion postulates that STAI-Trait scores and BDI scores share a common factor, negative affectivity, and Bieling et al. (1998) found that the STAI-Trait also contains a depression factor, also in common with the BDI. The components of these self-report inventories can be expressed as: 
STAI score $=$ General negative affectivity + Depression factor + Anxiety factor

BDI score $=$ General negative affectivity + Depression factor

It is apparent that on average individuals with high BDI scores will also have high STAI scores, and individuals with low BDI scores will also have low STAI scores. How could two groups with equal STAI scores, but high and low BDI scores, be generated? Assuming the equations above, the only mathematical possibility is this:

Low BDI group: Medium STAI score = Low depression and general negative affect + high anxiety.

High BDI group: Medium STAI score = High depression and general negative affect + low anxiety.

Thus, paradoxically, by selecting two groups that score equally on an anxiety inventory, Lawson and MacLeod (1999) have selected two groups that differ in level of pure anxiety. It is possible that a design similar to that used in their study would produce an identical set of seemingly puzzling results in a more traditional test of interpretative bias, such as the homophone interpretation task. Therefore, there is no need to conclude that the differences between the findings of their study and the findings of homophone interpretation studies are due to the presence of response biases in earlier studies and their absence in Lawson and MacLeod's (1999) study; rather, these unusual findings are 
explicable solely in terms of methodology. According to this view, anxiety causes negative interpretative bias, but pure depression does not affect interpretative bias either way. The view predicts that clinically depressed individuals, being high in anxiety, will show a strong negative interpretative bias. The implications of this view are in strong contrast to the conclusions of the authors of this study, who call on clinicians and researchers to consider the possibility that depressed people are not negatively biased in their interpretation of the world at all, but instead show a strong negative response bias.

In summary, research on depression and anxiety has found that these emotions are related to negative interpretative biases. There is evidence that for anxiety at least, this interpretative bias cannot be solely attributed to response bias. There has been little research contrasting the effects of anxiety and depression, but the two studies reviewed that did perform this contrast found that, of the two emotional states, anxiety seems to be the more closely related to interpretative bias.

\section{Theories of Information Processing in Anxiety and Depression}

As discussed earlier, research on information processing in anxiety and depression was originally inspired by Beck’s cognitive model of depression, and much research in this area continues to be conducted with the explicit goal of testing aspects of this theory. It is therefore appropriate to consider the theoretical mechanism that Beck’s theory postulates to explain negative information processing biases. The most relevant component of Beck's theory is the idea of a schema (Gotlib \& MacLeod, 1997). A schema is a hypothetical 
cognitive structure that organises information about a topic. Schemas can be held about many aspects of the world, and it is postulated that they affect attention, judgement, and memory. For example, you could have a dishwashing schema that serves to direct your attention to the dishwashing detergent bottle, and to judge that the yellow liquid within is detergent and not mustard. Later when you are at the supermarket trying to remember what you ran out of when you were doing the dishes, your dishwashing schema would help remind you that you finished all the detergent. Some schemas, like that of a dishwashing schema, are held by most people. Other schemas may be highly developed in some people, but may be less developed or absent in others. For example, expert chess players, novice chess players, and people who have never played chess will have different schemas regarding the pattern of pieces on a chess board. These different schemas affect the way that people with differing levels of experience remember chess games (Gilhooly \& Green, 1988). Similarly, Beck et al. (1979) hypothesised that anxious and depressed individuals hold schemas that serve to enhance processing of either loss or threat information. They speculated that these negative schemas may be formed in childhood, and also suggested that they are only activated under certain circumstances. Importantly, schema theory suggests that anxiety and depression influence cognition in essentially the same way - through the activation of relevant schemas. This implies that although schema content may differ between anxious and depressed people, both groups will show negative biases in attention, judgement, and memory (Williams et al., 1988).

Another highly influential theory is the network theory put forward by Bower (1981). Network theory suggests that representations can be thought of as 
nodes within a network. Connections between nodes (representations) can be strong or weak, and concurrent activation of nodes tends to strengthen the connections between them. The activation of one node in the network tends to spread to other, strongly connected nodes, with the result that they are more easily activated by subsequent stimuli. Bower also postulated “depressed mood” and "anxious mood" nodes within the network. Because activation of depressed mood is likely to frequently co-occur with activation of representations of loss, strong associations between depression and representations of loss are likely to form. Thus, activation of depressed mood would tend to activate representations of loss. This means that external loss related material would be more likely to be initially activated (attended to) and subsequently reactivated (remembered) when the depression node was active. Similarly, activation of the depression node would make it easier for ambiguous material to activate loss-related representations. Bower postulated a similar relationship between the anxiety node and representations of threat related material. Because anxiety and depression nodes are thought to influence cognitive processes through essentially the same mechanism, the theory predicts that depression and anxiety may have content-specific effects, but will both bias attention, memory, and judgement (Bower, 1981). Thus Beck’s schema and Bower’s network theories are very similar in their predictions, and could be thought of as different ways of expressing the same basic idea. In fact, they may be compatible enough to be integrated into a single theoretical framework; Horowitz et al. (1997) have put forward a theory that conceives of a schema as a strongly associated network of representations. 
Although plausible, schema and network theories of information processing biases suffer from a problem that was first pointed out by Williams et al. (1988). As already discussed, both schema and network theories predict that anxiety and depression should both produce negative processing biases across the three domains of attention, memory, and judgement. As was already apparent at the time of the literature review conducted by Williams et al. (1988), anxiety and depression seem to have different effects on these domains. The major reviews conducted by MacLeod and Rutherford (1998), Williams et al. (1988), and Williams et al. (1997) have all found that depression seems to be more consistently associated with biases in explicit memory, and anxiety seems to be more consistently associated with biases in implicit memory and attention. Williams et al. (1997) also speculated that biases in judgement might be more closely related to anxiety than to depression, and the current review found some preliminary evidence for this proposition. The differing information processing correlates of anxiety and depression led Williams et al. (1988) to propose a revised theory of information processing biases. They referred to earlier experimental work on memory that distinguished between two distinct processes - priming and elaboration. Priming is the strengthening of connections within a representation, and makes that representation more easily activated. Elaboration is the strengthening of connections between representations, and this process makes the target representation easier to retrieve deliberately. Priming is the principal mechanism of perceptual implicit memory and the disambiguation of stimuli, and it also affects attentional processes. Elaboration is the principal mechanism of explicit memory. Williams et al. (1988) postulated that anxiety predominately biases priming, and depression predominately biases elaboration. 
This means that anxiety will affect processes that are based on priming, such as attention and perceptual implicit memory. It also means that depression will affect processes that are based on elaboration, such as explicit and autobiographical memory.

Williams et al.'s (1988) theory neatly explained the data that existed at that time. Williams et al. (1997) conducted a major narrative review of the literature subsequent to their earlier book, partially with the intention of evaluating their earlier theory. Their review was broadly supportive of their general model, but also found some evidence inconsistent with their model. Williams et al. (1997) discussed possible reasons for this, and proposed some extensions to their theory. In particular, they suggest that in addition to the distinction between priming and elaboration, there is an additional distinction between memory-related and non-memory-related processes. They also elaborated on how their model could be accommodated within two broader theories of memory, emotion, and cognitive processing: the multiple-entry memory system (MEM; Johnson \& Multhaup, 1992) and the theory of interacting cognitive subsystems (ICS; Teasdale \& Barnard, 1993). With the possible exception of the findings of studies using the DOAT task to measure attention in depression, the recent experimental literature as reviewed in this thesis remains consistent with the revised model put forward by Williams et al. (1997). However, as acknowledged by these authors, both their own theory and the theories of Johnson and Multhaup (1992) and Teasdale and Barnard (1993) remain reasonably broad in their predictions. The refinement of these models is dependent on further research on cognition and emotion, and one particularly promising path is further specification of the distinctions between cognitive 
processes in different emotions (Williams et al., 1997). This thesis contributes to this development by contrasting the cognitive processes in the related emotions of anxiety and depression.

\section{$\underline{\text { Somatic and Cognitive Aspects of Depression and Cognitive Biases }}$}

It is common for cognitively oriented researchers to attempt to explain cognitive biases in depression without reference to biological mediation. For example, in Bower's (1981) model, mood-congruent memory in depressed individuals is explained by stronger connections between nodes that code for negative information. Pure-cognitive models of depression and information processing predict that negatively biased information processing will be most strongly associated with those items on depression inventories that measure depressed thoughts and beliefs, rather than those measuring somatic symptoms. Horowitz et al. (1997), who propose a pure-cognitive model of depression, explicitly argue that information processing biases are tied to psychological rather than biological variables, and suggest that information processing biases will be altered more by psychological treatments than by pharmacological treatments.

Close consideration of pure-cognitive models of mood-congruent information processing in depression reveals that they are inconsistent with some of the evidence on cognition and emotion. Most tellingly, Kumari et al. (1998) investigated the effects of placebo and of the mood-lowering drug haloperidol on recall of autobiographical memories. They found that the drug caused participants to recall unhappier memories, and that the change in valence of 
recalled events was only partially mediated by self-rated mood. This study suggests that negative biases in recall are strongly biologically mediated. Also consistent with this conclusion is the study of Clark and Teasdale (1982), who investigated valence of autobiographical events recalled at different times of the day. The participants were depressed individuals with strong diurnal variation in mood, and this variation is widely believed to be biological in nature (Shelton, Hollon, Purdon, \& Loosen, 1991). Valence of recalled events varied with time of day in a manner that was congruent with current mood. Deldin, Keller, Gergen, and Miller (2001) have found that differences in information processing between depressed and nondepressed individuals are detectable using event-related brain potentials. The fact that differences in processing are detectable using gross neuropsychological measurements is more consistent with biological involvement than with pure-cognitive theories. As noted earlier, the theories of Williams et al. (1997) and Teasdale and Barnard (1993) are amongst the most successful in explaining the current experimental literature on cognition in emotion. Both of these theories - especially the ICS theory (Teasdale, 1999) argue that the cognitive mechanisms they postulate will ultimately be explicable in neurobiological terms. Rolls and Treves (1998) take the first steps in formulating such an explanation by suggesting specific neural pathways that could mediate mood-congruent processing. These proposed biologically mediated models of selective information processing are consistent with proposals that information processing in anxiety and depression serves an evolutionarily designed purpose (Mathews, 1990). Models that suggest neurobiological mediation of the relationship between emotion and cognitive processes predict that information processing biases will be more closely 
associated with the somatic symptoms of depression than with the cognitive symptoms. The current studies investigate the relative influence of somatic and cognitive symptoms of depression in order to determine whether moodcongruent information processing is biologically mediated.

\section{Using Information Processing Biases to Measure Depression}

As well as the theoretical reasons for investigating information processing biases, there has been some speculation that their measurement might be of practical use. Horowitz et al. (1997) proposed that a participant's mean negative bias score across a battery of information processing tasks could be used to assess their level of depression. In theory, an information processing battery would eliminate the effect of conscious response strategies on the measurement of depression, and this would have a variety of practical benefits (Horowitz et al., 1997). For example, results on a well-designed information processing battery would be free of demand effects, making it a more accurate index of improvement with treatment. Similarly, the battery could be used to compare males with females, without the potentially contaminating influence of different degrees of self-disclosure. Because testing the main hypotheses of this thesis involved implementing batteries of information processing tasks, many of which are thought to relate to some degree to depression, the project was able to test the practicality of the idea of an information processing measure of depression. 
Hemispheric Thinking Style and Information Processing

This introduction has discussed the influences of depression and anxiety on information processing. Another variable that may influence information processing is hemispheric thinking style. The idea that people may show thinking styles related to hemispheric dominance arose from evidence that the two hemispheres of the brain show some specialisation of function (Iaccino, 1993). This evidence for functional differences was exaggerated and distorted by the popular press, as was the idea that some people might rely more heavily on one hemisphere than on the other (Corballis, 1999). However, despite the plethora of misleading information, recent evidence is consistent with the idea that there are some functional differences between the left and right hemispheres (Iaccino, 1993). Further, there is evidence for stable individual differences in patterns of activation of the left and right hemispheres, and these differences correlate with answers to a paper and pencil thinking style questionnaire (Merckelbach, Muris, Pool, \& De Jong, 1996). This suggests that people do show different thinking styles, and that these thinking styles are influenced by differences in brain function.

This thesis examines two aspects of the influence of thinking style on information processing. First, Merckelbach, Muris, and Horselenberg (1996) examined the effect of thinking style on ability to produce specific memories. Their investigation was based on Iaccino's (1993) argument that the left hemisphere is specialised for analytic and verbal thinking, and the right hemisphere for holistic and nonverbal thinking. They predicted that people with a right-hemispheric dominant thinking style would pay less attention to detail and 
would thus be more likely to produce overgeneral memories (Merckelbach, Muris, \& Horselenberg, 1996). They obtained results opposed to their prediction: right-hemispheric thinking style was linked to the production of fewer, rather than more, overgeneral memories. The theory behind their prediction is also of uncertain validity, in that the hypothesis of hemispheric specialisation for analytic and holistic thinking has not received clear empirical support (Coren, 1992). Nonetheless, because some authors have suggested that overgeneral recall may predispose people to depression (Williams, 1992), potential influences on memory specificity are of relevance to research into depression. Experiment 2 includes a partial replication of Merckelbach, Muris, and Horselenberg's (1996) investigation; more detail on this matter is included in the discussion of Experiment 1.

Second, there is evidence that the right-hemisphere is specialised for the processing of emotion-related information (Borod, Bloom, Brickman, Nakhutina, \& Curko, 2002; Mandal, Asthana, Pandey, \& Sarbadhikari, 1996). Thus, individuals with a right-hemispheric dominant thinking style might be more likely to interpret ambiguous information in an emotional way. Experiment 2 tested this hypothesis.

\section{$\underline{\text { The Purpose of the Current Investigation }}$}

This review discussed the historical context of the study of anxiety, depression, and information processing bias. Then three methodological issues were discussed - anxiety and depression as uncontrolled confounds of each other, clinical versus nonclinical samples, and statistical power. The literature relating to emotion and information processing was reviewed, highlighting 
examples of these methodological problems and established differences between the information processing correlates of anxiety and depression. The main theories that seek to explain information processing biases in depression and anxiety were reviewed, and the importance to theory of differences in the information processing correlates of anxiety and depression was highlighted. In essence, Beck's schema theory, Bower's network theory, and these theories' derivatives predict that anxiety and depression will both be characterised by pervasive negative biases. In contrast, Williams et al.'s $(1988,1997)$ theory predicts that anxiety will have a greater influence on tasks involving priming, and depression will have a greater influence on tasks involving elaboration. Finally, the explicit or implicit assumption of cognitive theorists that cognitive biases are the product of purely cognitive rather than biologically based processes is contrasted with existing empirical evidence and opposing theoretical viewpoints.

The current studies examine the two issues discussed in the final sections on theory, with reference to the methodological issues also discussed. The two major aims were:

1. To test the hypothesis of Williams et al. $(1988,1997)$ that anxiety is associated with cognitive biases in tasks requiring priming, and depression is associated with cognitive biases in tasks requiring elaboration.

2. To test the hypothesis, derived from the current literature review, that cognitive biases in depression are more closely related to the somatic than to the cognitive aspects of depression. Usefully, the Beck 
Depression Inventory - II possesses separate cognitive-affective and somatic factors (Dozois, Dobson, \& Ahnberg, 1998), and the experiments described investigate which of these factors is more closely associated with information processing biases.

A variety of studies have already been conducted that are relevant to Aim 1, but they were not explicitly designed to address this issue. Williams et al. (1988, 1997) acknowledge that in the existing research literature, betweenexperiment methodological differences are an important limiting factor on the precision with which comparisons of the effects of anxiety and depression can be made. The current research is a direct test of Williams et al.'s $(1988,1997)$ hypothesis, and thus it has several methodological advantages. First, the experiment consistently measured both anxiety and depression, using scales that clearly discriminate between the two emotions. This meant that their effects could be directly compared, holding experimental methodology constant. It also allowed partial correlations to be conducted, allowing the experimenter to examine whether either depression or anxiety has an effect that is independent of the other emotion. Second, tasks reliant on both priming-dependent and elaboration-dependent processes were included within each experiment, allowing the two types of processes to be directly compared.

It is also worth summarising the subsidiary aims of the project, discussed in detail in the earlier literature review:

1. Experiment 1 examined whether positive biases on the deployment of attention task were shown by the majority of the population, or 
whether these biases were shown only by those who were unusually low in depression and anxiety.

2. Experiment 2 examined several influences on the specificity of autobiographical memory.

3. Experiment 2 also examined the effect of left- and right- hemispheric thinking style on the interpretation of emotional material.

4. The project examined the viability of using information processing tasks to assess level of depression.

\section{$\underline{\text { Hypotheses of Experiment } 1}$}

Experiment 1 contained two elaboration-dependent and three primingdependent tasks adapted from the existing cognitive experimental literature. Tasks were chosen based on degree of existing empirical support, and on the basis that their mood-related nature would not be apparent to the participants, so that the results would be free from demand effects. The elaboration-dependent tasks were an explicit memory task and a conceptual implicit memory task, the latter based closely on Watkins et al. (1996). It was hypothesised that bias on these tasks would be directly associated with depression, but not directly associated with anxiety. The priming-dependent tasks were a perceptual implicit memory task, a test of emotion-related attentional interference (the emotional Stroop), and a test of emotion-related attentional facilitation (the DOAT). It was hypothesised that bias on these tasks would be directly associated with anxiety, but not directly associated with depression. Finally, the subliminal priming task implemented by Bradley et al. (1994) was also included, but post-experimental 
checks showed that the priming part of this task was not implemented with the necessary methodological fidelity, and so this task will not be further discussed here.

Thus, the hypotheses of Experiment 1 were:

\section{$\underline{\text { Relevant to Aim } 1}$}

1. Negative bias scores derived from the explicit memory task would correlate positively with depression scores, but less strongly with anxiety scores.

2. Negative bias scores derived from the conceptual implicit memory task would correlate positively with depression scores but less strongly with anxiety scores.

3. Negative bias scores derived from the perceptual implicit memory task would correlate positively with anxiety scores, but less strongly with depression scores.

4. The interference scores derived from the emotional Stroop task would correlate positively with anxiety scores, but less strongly with depression scores.

5. The negative bias scores derived from the DOAT would correlate positively with anxiety scores, but less strongly with depression scores.

\section{$\underline{\text { Relevant to Aim } 2}$}

6. When negative bias scores correlated significantly with depression scores, they would correlate more strongly with the somatic factor of the inventory used than with the cognitive-affective factor.

\section{Relevant to Subsidiary Aim 1}

7. Consistent with the assumptions of Gotlib et al. (1988) and subsequent authors, those participants in the lower three quartiles of anxiety and depression would show a positive bias on the deployment of attention task; only those 
Emotion-Congruent Information Processing 56

participants scoring in the highest quartile of these measures would show unbiased attention. 
Experiment 1

Method

\section{$\underline{\text { Overview }}$}

Undergraduate students completed six computer-administered tasks designed to measure mood-congruent bias. Three of these tasks examined memory: a perceptual implicit memory test (word-stem completion), a conceptual implicit memory test (free association), and an intentional recall task. These tasks compared responses to negative and positive stimuli, and the dependent variables were the number of positive and negative words recalled. The other three tasks compared responses to negative and neutral stimuli. One task was the emotional Stroop, for which the dependent variable was the amount of emotional Stroop interference. Another was a primed lexical decision task, for which the dependent variables were the magnitudes of the priming that occurred for negative and neutral words. The final task was the deployment of attention task (DOAT), for which the dependent variable was the proportion of trials in which the participant said that the coloured bar that replaced the negatively valenced word appeared first. Following the experimental tasks, the participants completed self-report measures of depression and anxiety. 


\section{$\underline{\text { Participants }}$}

Participants were 203 Victoria University of Wellington students, recruited using notices placed around campus. They received a small monetary reward to compensate them for their time. Supplying demographic data was optional, but of those who answered, 92 were female and 109 were male. The median age of the participants was 21 years. There were 154 New Zealand European participants, 11 New Zealand Maori, and 32 of other ethnicity. There were 42 participants who had taken a university psychology paper at some stage, and 161 who had not. Potential participants were excluded from the experiment if they had started or completed a third year of study in psychology or if English was not their first language.

\section{$\underline{\text { Materials }}$}

Depression and anxiety were measured with the Beck Depression Inventory - II (BDI-II; Beck, 1996) and the Hospital Anxiety and Depression scale (HAD; Zigmond \& Snaith, 1983). The original Beck Depression Inventory has excellent psychometric properties and is widely recommended (Basco, Krebaum, \& Rush, 1997), and there is evidence that this inventory has been further improved in the second edition (Beck, 1996). The HAD also shows good psychometric properties, and has been widely used (Bjelland, Dahl, Haug, \& Neckelmann, 2002; Herrmann, 1997). Several studies have shown that the HAD adequately differentiates anxiety and depression (Bjelland et al., 2002). Although the HAD was developed in a hospital setting, it is often used in other settings (for example, 
Bradley et al., 1996), and the authors of the scale endorse this use (Snaith, 1992). Consistent with convention, both subscales of the HAD were administered. However, to prevent unnecessary duplication, only the anxiety subscale (the HAD-A) was included in the analyses.

\section{Apparatus}

The experimental computer program was custom designed in Visual Basic, and is available on request. The program was run on Pentium III computers with standard colour CRT monitors, under Windows 98.

\section{Procedure}

The introduction to the study and the encoding and retrieval stages of the implicit memory tests were conducted before the other tasks, in the order presented below. The remaining four tasks were presented in randomised order. It was not possible to counterbalance the implicit memory tasks, because this would have allowed the possibility of the word stimuli used in the other experimental tasks contaminating performance in the implicit memory tasks.

After the participants had finished the computerised tasks, the computer administered the HAD, and the experimenter administered the BDI-II.

Introduction to the study. The participants were told that the general aim of the research was to look at the relationship between performance on different tests of attention and memory and recent experiences and feelings, and that the precise objectives of the experiment would be explained after the experiment. 
They then gave their informed consent. No further explanation of the purposes of the tasks was given during the experiment.

Participants were tested in groups of one to twelve individuals. Participants were seated in front of their own computer. The room was laid out such that individual responses were not easily visible to other participants. Participants were instructed by the experimenter to fill out a questionnaire asking for basic demographic data, to wear the headphones supplied, to follow the instructions given by the computer with great care, and to ask the experimenter for help immediately if they should become confused by any aspect of the procedure. The experimenter was available and in the room throughout the duration of the experiment. Instructions on the individual tasks were displayed on the computer screen. Binary or Likert scale choices were made using the computer keyboard, and whole word responses were made in a booklet supplied by the experimenter.

The perceptual and conceptual implicit memory tests. In outline, participants were asked to elaboratively encode a list of positive and negative words, and then their implicit memory for these words was tested with word stem completion and word association tasks.

The procedures for these tasks were based on the perceptual and conceptual implicit memory tasks used by Ruiz-Caballero and Gonzalez (1994), and Watkins, Vache, Verney, and Mathews (1996), respectively.

Encoding task. Word stimuli for the task were identical to those shown in Watkins et al. (1996), except that the neutral words were omitted. The word stimuli were divided into two non-overlapping sets, each with 8 negative and 8 positive words. The computer program randomly assigned participants to word set. The assigned word set was the studied word condition, and the non-assigned 
word set was the unstudied word condition. After a two second display of the prompt “Imagination task now begins”, the assigned word set was displayed.

Each word was presented individually for 15 seconds. Participants were asked to imagine themselves in a scene involving each word for the duration of the word presentation. Participants were then asked to answer the following questions about the scene imagined:

1. Was the scene an actual scene from your past or an imaginary scene?

2. Were you the principal character of the scene?

3. Rate the vividness of the scene from 1 to 9 by pressing the corresponding key

4. Rate the pleasantness of the scene from 1 to 9 by pressing the corresponding key

The next word was then presented. No practice trials were given. Following the imagination task, participants completed a brief distracter task. Participants were presented with arrays of lower case letters and asked to quickly decide whether there was an $m$ in each array. The computer program was set up to continue presenting trials for thirty seconds.

Perceptual implicit retrieval task. Participants were told to turn to the page in their experimental booklet which listed the first three letters of each of the words from the studied and unstudied word sets, for a total of 32 word stems, presented in alphabetical order. They were told to complete each word stem with the first word that came to mind, to complete the task as quickly as possible, and that they were being timed.

Conceptual implicit retrieval task. Participants were told that they were to take part in a free association task, and that they were to produce as many words 
as possible in response to some cue words. The computer presented them with cue words corresponding to each of the words from the studied and unstudied word sets, for a total of 32 cue words. The cue words were taken from Watkins et al. (1996). In contrast to Watkins et al. (1996), who recorded responses on a tape recorder, responses were written into the supplied booklet. Participants were given 20 seconds to respond to each cue word, followed by a tone and the immediate onset of the next cue word. Positive and negative cues were alternated.

The remaining four tasks were presented in randomised order.

The intentional recall task. Participants were told that they would be given four minutes to learn a word list, and that they would be asked to recall the words later in the experiment. They were then presented with 20 words (10 negative and 10 positive). The negative words have been normed and used in previous experiments on cognition and emotion (Bradley \& Mathews, 1983; Watkins et al., 1996). The positive words were derived by selecting negative words that were used by these sources, but that were not used for the current task, and determining their antonyms using a thesaurus (Urdang, 1997). The words were presented concurrently, positioned in individually randomised order. Following the learning procedure, the participants performed the m-search distracter task for three minutes, and then recalled as many words as they could within a three minute time limit. Responses were written into the supplied booklet.

The emotional Stroop task. Participants were told that they would be presented with some words, and that their task was to identify the colour that each word was presented in. The words could be presented in red, yellow, green, or blue. Participants identified the colour that each word was presented in using 
corresponding keys from the computer keyboard. Participants were first given 10 neutral word practice trials. Then, in the main task, 50 depression-related and 50 neutral words were presented. The depression-related words were from the same pool as used in the intentional recall task, but did not overlap with them. The neutral words were from Jenkins, Russell, and Suci (1958), Mathews, Mogg, May, and Eysenck (1989) and Watkins et al. (1996). Feedback on correctness was given after each trial. The computer recorded the number of words of each stimulus type correct, and reaction times for both correct and incorrect responses.

The primed lexical decision task. Participants were told that they would be presented with some strings of letters, and that they were to determine whether or not the letter strings formed valid words. Participants responded using the computer keyboard. Stimuli were the set of 50 depression-related and 50 neutral words from the Stroop task, and 100 pronounceable nonwords. Unfortunately, due to technical problems with the subliminal priming of the stimuli, the data from this task had to be discarded.

The deployment of attention (DOAT) task. Participants were told that in each trial, they were to fixate on a crosshair while being presented with two coloured bars, and that their task was to determine whether the green or the red bar appeared on the screen first. In fact, in every trial both bars appeared simultaneously. The bars were $16 \mathrm{~cm}$ apart for the first 101 participants, and 8 cm apart for the remaining 103 participants; thus only the latter set of participants viewed the stimuli within foveal vision. Each coloured bar replaced a word stimulus. In previous research, judging that a bar appeared first is taken to be an indication of attention to that word (McCabe \& Gotlib, 1995). Participants first completed six practice trials, in which both bars replaced neutral words, then 
fifty data collection trials, in which one bar replaced a neutral word and one bar replaced a negative word. The word stimuli used in the main task were identical to those used in the Stroop.

Administration of the mood questionnaires. After the participants had finished all of the experimental tasks, the computer prompted them to complete two final questionnaires. The first, the HAD, was administered by the computer. The computer then prompted the participant to ask for the BDI-II, which for copyright reasons was administered in written form. Participants were given the option of not answering some or all questionnaire items. Participants were then debriefed, and asked not to talk to other students about the purpose of the experiment.

\section{Results}

The experimenter was blind to responses to the emotion measures while scoring task responses, and while making decisions about excluding participants from analyses. Exclusion criteria were determined before the data were analysed. All t-tests for planned comparisons are one-tailed unless otherwise specified. This decision was made before the analyses were conducted. Since its inception, the use of directional statistical tests has had both advocates and detractors (for reviews, see Hsu, 2000; Leventhal, 1999; Lipsey, 1998; Overall, 1990). In this experiment, one-tailed tests were used because the comparisons conducted were direct replications of existing studies, and because lack of statistical power is a significant methodological problem in this area. The main hypotheses under investigation were whether mood-congruent effects, the existence of which has 
already been accepted in the literature, are associated with anxiety or with depression, and with cognitive or biological symptoms. Exact probabilities are also reported and can be used to calculate two-tailed probabilities if desired. All power calculations were conducted using the GPOWER computer program (Erdfelder, Faul, \& Buchner, 1996).

Exclusions for Poor Data Quality.

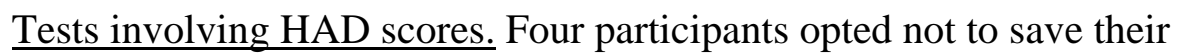
HAD results. All participants chose to complete the BDI-II.

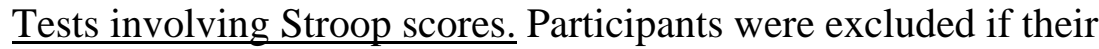
combined error score across both conditions was classified as an outlier by box plot analysis (more than 9 errors out of a total of 100 trials; seven participants were excluded).

Tests involving attention deployment scores. Due to technical problems with the administration of this task (the stimuli were placed too far apart for the words to be readable), the results for the first 101 participants are not presented. Participants were also excluded if the proportion of times they chose the top stimuli exceeded $90 \%$ or did not reach $10 \%$ (indicating they spent most of their time pressing only one key). This resulted in the exclusion of seven participants. A box-plot analysis was also conducted on the mean of the participants' median reaction times for the two word valences, but no low outliers were found. One participant was excluded for having a mean of median reaction times of less than the arbitrary but pre-determined cut-off of 250ms. These exclusion criteria meant that 93 participants were included in the analysis of this task. 
Tests involving implicit memory scores. Participants were excluded for not correctly completing the m-search task that immediately preceded the relevant implicit memory task. In practice, this meant calculating an m-search score for each participant, by subtracting number of incorrect answers from number of correct answers. If a participant's m-search score was classified as a low outlier, that participant was excluded. Nine participants were excluded from the word-stem completion task (for scoring below 15) and six participants were excluded from the word association task (for scoring below 20).

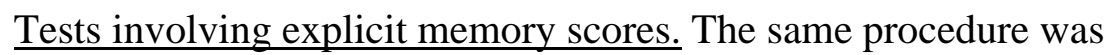
followed as for the implicit memory tasks. Five participants were excluded for scoring less than 57.

Lexical decision task. Due to technical problems with the priming of the stimuli, the results of this task are not presented.

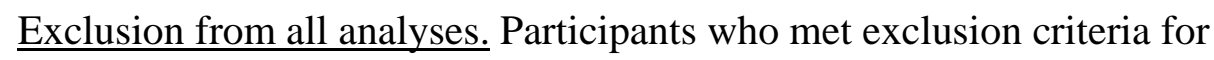
three or more task analyses may have been uncooperative. Three participants were excluded from all analyses for this reason.

\section{Depression and Anxiety Measures}

The mean BDI-II score was $10.27(\underline{\mathrm{SD}}=7.13)$, which is similar to that found in the normative sample reported in the inventory’s manual (Beck, 1996). The mean HAD-A score was 8.54 ( $\underline{\mathrm{SD}}=3.44)$, which is somewhat higher than the mean scores obtained by Spinhoven, Ormel, Sloekers, and Kempen (1997) for adults aged 18 to $65(\underline{\mathrm{M}}=5.1, \underline{\mathrm{SD}}=3.6)$. However, the same authors note a 
small negative correlation between age and HAD-A score. Thus, the young age of the present sample may partially explain its high mean HAD-A score.

\section{Division of Participants into Groups}

Anxiety and depression are often viewed as continuous variables (Ruscio \& Ruscio, 2000), especially within nonclinical populations. Using continuous variables to divide participants into arbitrary groups destroys information and detracts from statistical power (Nunnally \& Bernstein, 1994). Presenting the data as correlations avoids this problem and also has the advantage that the sizes of relationships can easily be assessed and compared. Thus, the primary analyses were t-tests of correlations between the emotion measures and the dependent variables. However, when significant correlations occurred, group analyses were conducted to allow absolute values of the dependent variables to be compared. For this purpose, participants were divided into groups based on the BDI-II ranges suggested by Dozois, Dobson, and Ahnberg (1998). These authors made their recommendations to preserve comparability to research using the widely cited (Haaga \& Solomon, 1993) BDI-I cutoff scores recommended by Kendall, Hollon, Beck, Hammen, and Ingram (1987) for analogue depression research. Thus, participants were classified as nondepressed (BDI-II scores of 0 to 12 , $\underline{\mathrm{n}}=$ 139), dysphoric (scores of 13 to $19, \underline{n}=40$ ) or dysphoric-depressed (scores of 20 or over, $\underline{\mathrm{n}}=21)$.

Because there are no existing recommendations for division of participants into groups for analogue anxiety research, participants were simply divided into quartile groups according to their HAD-A score. Thus, participants 
were classified as low anxiety (HAD-A scores of 0 to $5, \underline{n}=41$ ), medium-low anxiety (scores of 6 to $8, \underline{n}=54$ ), medium-high anxiety (scores of 9 or $10, \underline{n}=$ 45), or high anxiety (scores of 11 or above, $\underline{n}=57$ ). By coincidence, the range of scores in the medium-high anxiety group corresponds to the range Zigmond and Snaith (1983) suggest represents possible emotional disorder, and the range of scores in the high anxiety group corresponds to the range Herrmann (1997) suggests corresponds to abnormal anxiety.

\section{$\underline{\text { Explicit Memory Task }}$}

The variable of interest was explicit memory bias score, which can be conceptualised as the difference between the number of negative words recalled and the number of positive words recalled. Rather than calculating a memory bias score by subtracting number of positive words recalled from number of negative words recalled, the relationship between the predictor variables and memory bias was calculated by conducting a correlation between the predictor variable and number of negative words recalled, while partialling out number of positive words recalled. This method of analysis results in superior statistical power, without increasing the chances of producing spurious findings (Cohen, 1988).

Table 1 shows that the predicted correlation between BDI-II score and memory bias score was significant $(\underline{\mathrm{r}}=.120, \underline{\mathrm{p}}<.05)$. Memory bias score was not significantly related to HAD-A score $(r=.090, \mathrm{p}>.10)$. Partial correlations between depression, anxiety, and bias score were nonsignificant, but the pattern 
of results was consistent with the prediction that depression mediated any relationship between anxiety and bias score.

The relationships of the somatic and cognitive factors of depression to bias score were also compared. This correlation was stronger for the scale’s somatic factor $(\underline{r}=.124, \underline{p}<.05)$ than for its cognitive-affective factor; the latter correlation did not reach significance $(\underline{\mathrm{r}}=.095, \underline{\mathrm{p}}<.10)$. The partial correlation of bias with somatic depression, controlling for cognitive depression, was not significant $(\underline{\mathrm{pr}}=.082, \underline{\mathrm{p}}>.10)$. The partial correlation of bias with cognitive depression, controlling for somatic depression, was also nonsignificant $(\mathrm{pr}=$ $.006, \mathrm{p}>.10)$. Although neither partial correlation is significant, the pattern of results is consistent with the prediction that somatic symptoms mediated any relationship between cognitive-affective symptoms and memory bias.

Figure 1 shows the size of the negative bias shown by individuals from the different depression classifications. The dysphoric group did not show significantly more negative bias than did the nondepressed group $(\underline{\mathrm{M}}=0.95, \underline{\mathrm{SD}}$ $=2.05$, vs. $\underline{\mathrm{M}}=0.84, \underline{\mathrm{SD}}=1.96, \underline{\mathrm{t}}[174]=.314, \underline{\mathrm{p}}>.10)$. However, the dysphoricdepressed group did show significantly more negative bias than the nondepressed group $(\underline{\mathrm{M}}=1.67, \underline{\mathrm{SD}}=1.43$, vs. $\underline{\mathrm{M}}=0.84, \underline{\mathrm{SD}}=1.96, \underline{\mathrm{t}}[32.90]=2.34, \underline{\mathrm{p}}<.05)$. 
Table 1.

Correlations Between Information Processing Bias Scores and Emotion Measures.

\begin{tabular}{|c|c|c|c|c|c|c|c|}
\hline Task & $\begin{array}{l}\text { Explicit } \\
\text { memory }\end{array}$ & $\begin{array}{l}\text { Conceptual } \\
\text { implicit } \\
\text { memory } \\
\text { (word } \\
\text { association) }\end{array}$ & & $\begin{array}{l}\text { Perceptual } \\
\text { implicit } \\
\text { memory } \\
\text { (word } \\
\text { stem) }\end{array}$ & & $\begin{array}{l}\text { Emotional } \\
\text { Stroop }\end{array}$ & $\begin{array}{l}\text { Attention } \\
\text { Deploym- } \\
\text { ent }\end{array}$ \\
\hline $\begin{array}{l}\text { Emotion } \\
\text { measure }\end{array}$ & $\begin{array}{l}\text { Negative } \\
\text { words } \\
\text { recalled } \\
\text { (positive } \\
\text { words } \\
\text { recalled as } \\
\text { covariate) }\end{array}$ & $\begin{array}{l}\text { Primed } \\
\text { positive } \\
\text { target words } \\
\text { produced } \\
\text { (adjusted for } \\
\text { baseline) }\end{array}$ & $\begin{array}{l}\text { Primed } \\
\text { negative } \\
\text { target } \\
\text { words } \\
\text { produced } \\
\text { (adjusted } \\
\text { for } \\
\text { baseline) }\end{array}$ & $\begin{array}{l}\text { Primed } \\
\text { positive } \\
\text { target } \\
\text { words } \\
\text { produced } \\
\text { (adjusted } \\
\text { for } \\
\text { baseline) }\end{array}$ & $\begin{array}{l}\text { Primed } \\
\text { negative } \\
\text { target } \\
\text { words } \\
\text { produced } \\
\text { (adjusted } \\
\text { for } \\
\text { baseline) }\end{array}$ & $\begin{array}{l}\text { Harmoni } \\
\text { c mean } \\
\text { RT for } \\
\text { sad } \\
\text { words - } \\
\text { harmonic } \\
\text { mean RT } \\
\text { for } \\
\text { neutral } \\
\text { words }\end{array}$ & $\begin{array}{l}\text { Percentage } \\
\text { of trials } \\
\text { coloured } \\
\text { bar } \\
\text { replacing } \\
\text { sad word } \\
\text { selected }\end{array}$ \\
\hline BDI-II & $\begin{array}{l}\mathrm{pr}=.120^{* *} \\
\mathrm{p}=.047 \\
\underline{\mathrm{df}}=194\end{array}$ & $\begin{array}{l}\underline{\underline{r}}=-.041 \\
\underline{\underline{p}}=.285 \\
\underline{\mathrm{n}}=193\end{array}$ & $\begin{array}{l}\underline{\underline{r}}=.003 \\
\underline{\mathrm{p}}=.485 \\
\underline{\mathrm{n}}=193\end{array}$ & $\begin{array}{l}\underline{\mathrm{r}}=.126 \\
\underline{\mathrm{n}}=193\end{array}$ & $\begin{array}{l}\underline{\underline{r}}=-.009 \\
\underline{\mathrm{p}}=.453 \\
\underline{\mathrm{n}}=193\end{array}$ & $\begin{array}{l}\underline{\underline{r}}=.027 \\
\underline{\mathrm{p}}=.353 \\
\underline{\mathrm{n}}=193\end{array}$ & $\begin{array}{l}\underline{\underline{\mathrm{r}}}=.172^{* *} \\
\mathrm{p}=.050 \\
\underline{\underline{\mathrm{n}}}=93\end{array}$ \\
\hline $\begin{array}{l}\text { BDI-II } \\
\text { somatic } \\
\text { factor }\end{array}$ & $\begin{array}{l}\mathrm{pr}=.124^{* *} \\
\mathrm{p}=.042 \\
\mathrm{df}=194\end{array}$ & & & & & & $\begin{array}{l}\underline{\mathrm{r}}=.229^{* *} \\
\mathrm{p}=.014 \\
\underline{\mathrm{n}}=93\end{array}$ \\
\hline $\begin{array}{l}\text { BDI-II } \\
\text { cognitive- } \\
\text { affective } \\
\text { factor }\end{array}$ & $\begin{array}{l}\mathrm{pr}=.095^{*} \\
\mathrm{p}=.093 \\
\underline{\mathrm{df}}=194\end{array}$ & & & & & & $\begin{array}{l}\underline{\underline{r}}=.082 \\
\underline{\underline{p}}=.217 \\
\underline{n}=93\end{array}$ \\
\hline HAD-A & $\begin{array}{l}\mathrm{pr}=.090 \\
\mathrm{p}=.106 \\
\underline{\mathrm{df}}=191\end{array}$ & $\begin{array}{l}\underline{r}=-.029 \\
\underline{p}=.343 \\
\underline{n}=191\end{array}$ & $\begin{array}{l}\underline{\mathrm{r}}=.040 \\
\mathrm{p}=.291 \\
\underline{\mathrm{n}}=191\end{array}$ & $\begin{array}{l}\underline{\mathrm{r}}=.081 \\
\underline{\mathrm{n}}=190\end{array}$ & $\begin{array}{l}\underline{\mathrm{r}}=.189^{* * *} \\
\mathrm{p}=.004 \\
\underline{\mathrm{n}}=193\end{array}$ & $\begin{array}{l}\underline{\mathrm{r}}=-.058 \\
\underline{\mathrm{n}}=190\end{array}$ & $\begin{array}{l}\underline{r}=.195^{* *} \\
\mathrm{p}=.031 \\
\underline{n}=92\end{array}$ \\
\hline $\begin{array}{l}\text { Partial } \\
\text { correlation } \\
\text { with BDI-II } \\
\text { controlling } \\
\text { for HAD-A }\end{array}$ & $\begin{array}{l}\underline{\mathrm{pr}}=.083 \\
\mathrm{p}=.123 \\
\underline{\mathrm{df}}=190\end{array}$ & & & & $\begin{array}{l}\mathrm{pr}=-.199^{\psi} \\
\underline{\mathrm{df}}=187\end{array}$ & & $\begin{array}{l}\mathrm{pr}=.055 \\
\mathrm{p}=.303 \\
\underline{\mathrm{df}}=89\end{array}$ \\
\hline $\begin{array}{l}\text { Partial } \\
\text { correlation } \\
\text { with HAD-A } \\
\text { controlling } \\
\text { for BDI-II }\end{array}$ & $\begin{array}{l}\mathrm{pr}=.022 \\
\mathrm{p}=.381 \\
\mathrm{df}=190\end{array}$ & & & & $\begin{array}{l}\mathrm{pr}= \\
.270^{* * * *} \\
\mathrm{p}=.000 \\
\underline{\mathrm{df}}=187\end{array}$ & & $\begin{array}{l}\mathrm{pr}=.122 \\
\mathrm{p}=.125 \\
\underline{\mathrm{df}}=89\end{array}$ \\
\hline
\end{tabular}

$*$ indicates near significance $(\mathrm{p}<.10) . * *$ indicates significant at $\mathrm{p}<.05 . * * *$ indicates significant at $\mathrm{p}<.01 .{ }^{* * * *}$ indicates significant at $\mathrm{p}<.001$. All t-tests were one-tailed. $\mathrm{p}$ values for correlations in the direction opposite to that predicted are not reported as one-tailed t-tests have no statistical power in this circumstance. The use of two-tailed t-tests would have produced one additional significant finding, marked ${ }^{\psi}$, as noted in the main text. BDI-II factor correlations were calculated only when the BDI-II correlated significantly with the relevant variable. Partial correlations were calculated only when the BDI-II or HAD-A correlated significantly with the relevant variable. 


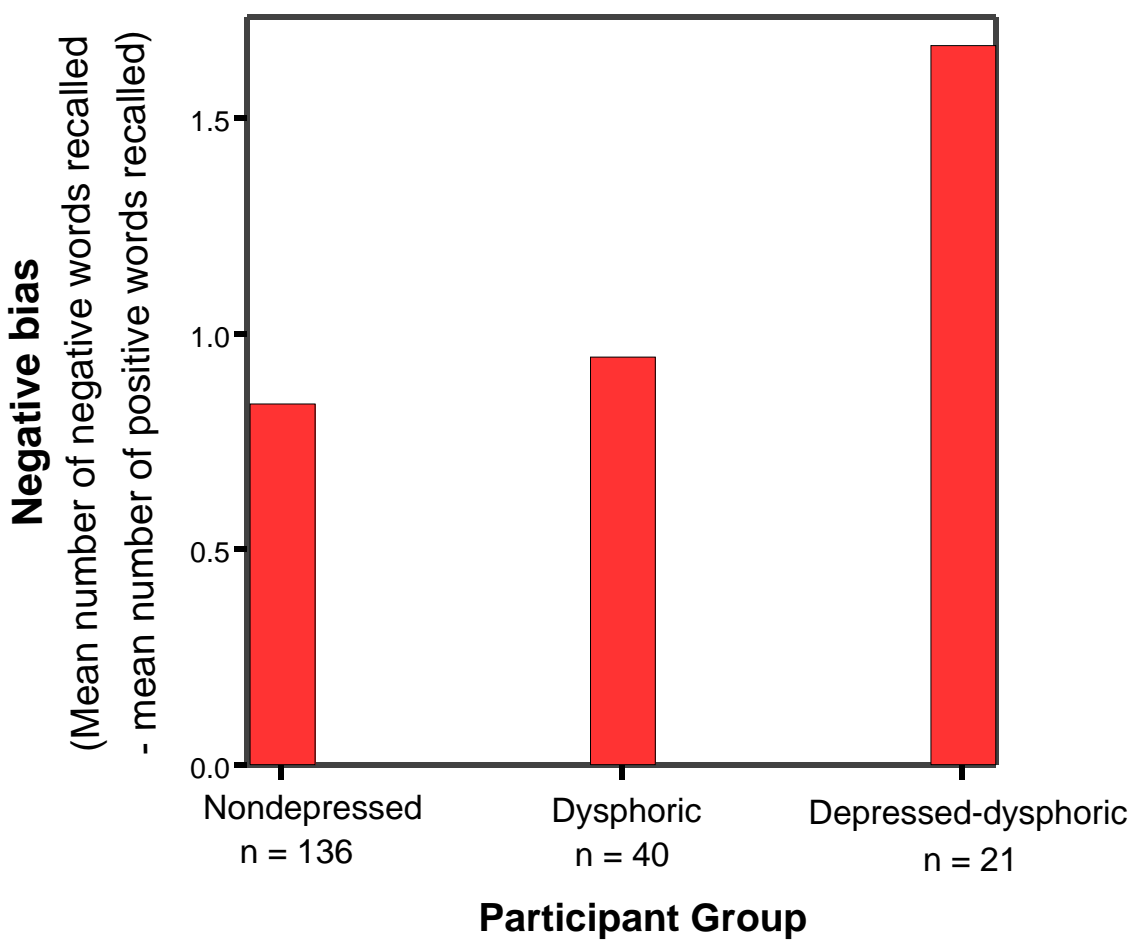

Figure 1.

Depression group and negative bias in explicit memory. 


\section{$\underline{\text { Word Stem Completion Task }}$}

So that correlational analyses could be conducted, an estimate of the amount of priming that was experienced by each individual participant was calculated. First, baseline performance for each word set was calculated; this is equal to the mean number of target words completed by participants for whom it was the unprimed word set. The number of target words completed by each participant in his or her primed condition was adjusted by subtracting the baseline number of target words produced in that word set. These calculations result in the adjusted for baseline number of positive and negative words produced. These are the dependent variables and are best-estimates of the amount of priming experienced by each participant ${ }^{2}$.

Table 1 shows that adjusted-for-baseline number of positive target words was not significantly correlated with either BDI-II or HAD-A score. Adjusted number of negative target words produced was not significantly correlated with the BDI-II. However, there was a significant correlation in the predicted direction between HAD-A score and adjusted number of negative target words produced $(\underline{r}=.189, \underline{\mathrm{p}}<.01)$. Moreover, this correlation increases in strength when BDI-II score is statistically controlled for $(\mathrm{pr}=.270, \mathrm{p}<.001)$. There is also an unpredicted negative correlation between BDI-II score and adjusted

\footnotetext{
${ }^{2}$ The results are similar when baseline is not controlled for. As predicted, most indices of unprimed performance were unaffected by mood. Adjusted for baseline number of unprimed positive target words produced did not correlate with either BDI-II score $(\underline{r}=.026, \mathrm{p}>.10)$ or HAD-A score $(\mathrm{r}=.077, \mathrm{p}>.10)$. Adjusted number of unprimed negative target words produced did not correlate with HAD-A score $(r=.067, \mathrm{p}>.10)$. However, adjusted number of unprimed negative target words produced did correlate with BDI-II score $(\underline{r}=.149, \underline{\mathrm{p}}<.05)$. This does not affect the priming analyses because as reported in the main text, adjusted number of primed negative target words produced did not correlate with BDI-II score.
} 
number of negative target words produced when HAD-A is statistically controlled for. This correlation was in the opposite direction to that predicted, but it should be noted that it would have been significant had a two-tailed t-test been conducted ( $\mathrm{pr}=-.199, \underline{\mathrm{p}}<.01$, two-tailed). This unexpected finding may be the result of the positive correlation between HAD-A score and number of negative target words produced.

Figure 2 shows the relationship between anxiety group and mean adjusted number of primed negative target words produced. The predicted difference between the top and bottom quartiles was significant $(\underline{\mathrm{M}}=1.20, \underline{\mathrm{SD}}=1.25$ vs. $\underline{\mathrm{M}}$ $=0.69, \underline{\mathrm{SD}}=1.23, \underline{\mathrm{t}}[94]=1.91, \underline{\mathrm{p}}<.05)$.

Finally, there was also a significant difference between the primed and unprimed conditions in adjusted-for-baseline number of words produced, for both the positive $(\underline{\mathrm{M}}=1.16, \underline{\mathrm{SD}}=1.39$ vs. $\underline{\mathrm{M}}=0.07, \underline{\mathrm{SD}}=0.93$, $\underline{\mathrm{t}}[192]=9.32$, $\underline{\mathrm{p}}$ $<.001)$ and negative $\left(\underline{\mathrm{M}}=1.09, \underline{\mathrm{SD}}=1.38\right.$ vs. $\underline{\mathrm{M}}=0.04, \underline{\mathrm{SD}}=1.11_{2} \underline{\mathrm{t}}[192]=$ 8.97, $\mathrm{p}<.001$ ) word sets. This shows that priming did take place. 


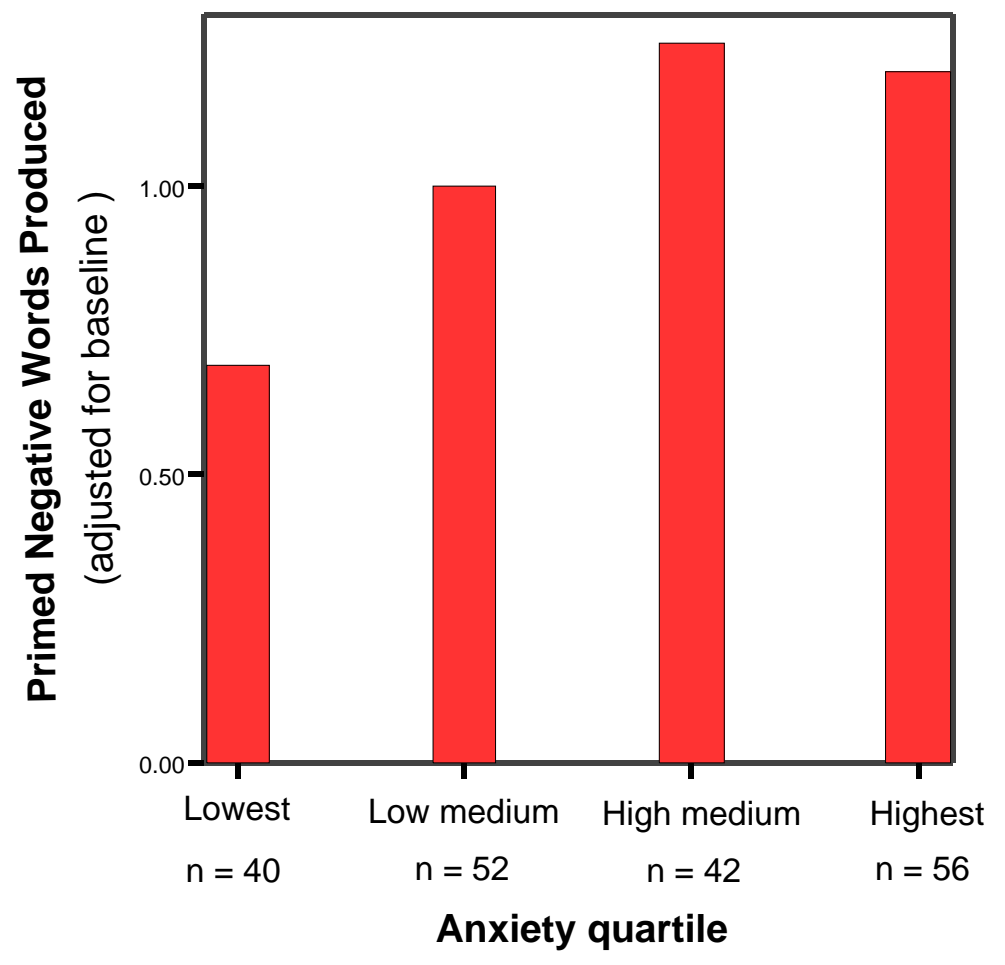

Figure 2.

Word stem task: priming of negative target words in each anxiety quartile. 


\section{Word Association Task}

The dependent variables in the word association task were calculated in an identical way to those in the word stem completion task. As Table 1 shows, neither adjusted for baseline positive nor adjusted for baseline negative target word production was related to either BDI-II or HAD-A score. Again, for the participants as a whole, adjusted for baseline primed number of target words produced was significantly greater than baseline for both the positive stimuli $(\underline{\mathrm{M}}$ $=0.81, \underline{\mathrm{SD}}=1.10$, vs. $\underline{\mathrm{M}}=0.02, \underline{\mathrm{SD}}=0.89 ; \underline{\mathrm{t}}[192]=7.63, \underline{\mathrm{p}}<.001)$ and the negative stimuli $(\underline{\mathrm{M}}=0.63, \underline{\mathrm{SD}}=1.23$, vs. $\underline{\mathrm{M}}=-0.04, \underline{\mathrm{SD}}=1.07 ; \underline{\mathrm{t}}[192]=6.12$, $\mathrm{p}$ $<.001)$.

A post-hoc power analysis was conducted to gain an idea of whether the failure of the experiment to find significant associations between BDI-II or HAD-A score and priming was due to insufficient statistical power. If the true $\underline{r}$ between priming and BDI-II or HAD-A was .15, then to obtain $90 \%$ power with 191 participants in a one-tailed correlational t-test would require setting alpha to .21. As Table 1 shows, none of the observed correlations were significant by this criterion. Although the post-hoc nature of these calculations calls for caution, the results indicates that insufficient power is a relatively unlikely explanation of the lack of significant relationships between the variables. 


\section{Emotional Stroop Task}

Reaction times less than 200ms or greater than 2000ms were considered outliers and removed from the data. Harmonic means, rather than means or medians, were calculated for each participant in each condition, because for reaction time data, this transformation produces a more reliable estimate of central tendency (Ratcliff, 1993).

Past research with the emotional Stroop task has been carried out using specialised response boxes, but in the present experiment standard PC keyboards were used. Consequently, split-half reliability was used to estimate the accuracy with which participant's response times were being measured. This was determined by calculating the correlation between the harmonic mean reaction time for each participant's odd-numbered trials and the corresponding harmonic mean reaction time for each participant's even-numbered trials. For the neutral stimuli, the split-half reliability was .921 , and for the negative stimuli, the splithalf reliability was .944 (both $\underline{\mathrm{p}}<.001, \underline{\mathrm{n}}=193$ ). By the Spearman-Brown prophecy formula (Spearman, 1910), the estimated reliabilities of the harmonic means of the complete set of reaction times to the neutral and negative stimuli are 0.959 and 0.971 , respectively. This demonstrates that reaction times were measured accurately enough for any relationship with the emotion measures to be revealed.

The dependent variable was the harmonic mean of each participant's reaction time to the negative stimuli, minus that participant's harmonic mean reaction time to the neutral stimuli. This was taken to be an index of Stroop interference caused by the negative stimuli. As Table 1 shows, this interference 
index did not correlate significantly with either BDI-II or HAD-A scores. For all participants combined, the value of the interference index did not significantly differ from $0(\underline{\mathrm{M}}=1.09 \mathrm{~ms}, \underline{\mathrm{SD}}=30.75, \underline{\mathrm{t}}[192]=0.49, \underline{\mathrm{p}}>.10)$, which means that participants were no slower to respond to the negative stimuli than they were to respond to the neutral stimuli.

A post-hoc power analysis was conducted to gain an idea of whether the failure of the experiment to find significant associations between BDI-II or HAD-A score and Stroop interference was due to insufficient statistical power. If the true $\underline{r}$ between priming and BDI-II or HAD-A was .15, then to obtain $90 \%$ power with 190 participants in a one-tailed correlational t-test would require setting alpha to .21. As Table 1 shows, neither of the observed correlations were significant by this criterion. Although the post-hoc nature of these calculations calls for caution, this results indicates that insufficient power is a relatively unlikely explanation of the lack of significant relationships between the variables.

\section{Attention Deployment Task}

The dependent variable was the number of times that the participant decided that the coloured bar that replaced the negative word appeared first, as a percentage of total trials. Table 1 shows that the predicted correlation between BDI-II score and percentage negative selected reached significance $(\underline{\mathrm{r}}=.172, \underline{p}=$ .05). As was the case with the explicit memory task, bias is more closely related to the somatic factor of the scale $(\underline{r}=.229, \underline{p}<.05)$ than to the cognitive-affective factor; the latter correlation is nonsignificant $(\underline{r}=.082, \underline{p}>.10)$. Partial 
correlations were consistent with these results. Controlling for the cognitiveaffective factor, the somatic factor was significantly associated with negative bias in attention $(\mathrm{pr}=.240, \mathrm{p}<.05)$, whereas the partial correlation between the cognitive-affective factor and negative bias, controlling for the somatic factor, was nonsigificant $(\underline{p r}=-.111, \underline{p}>.10)$. (Note that this finding cannot be explained by a closer association between the somatic factor and anxiety than between the cognitive-affective factor and anxiety, because in fact the somatic factor is somewhat less associated with HAD-A score than is the cognitiveaffective factor; $\underline{\mathrm{r}}=.513$ and $\underline{\mathrm{r}}=.578$, respectively, both $\underline{\mathrm{p}}<.001$ ). Also as predicted, HAD-A score is significantly correlated with percentage negative selected $(\underline{\mathrm{r}}=.195, \underline{\mathrm{p}}<.05)$. Neither BDI-II controlling for HAD-A nor HAD-A controlling for BDI-II correlates significantly with percentage negative selected $(\underline{\mathrm{pr}}=.055$ and $\mathrm{pr}=.122$, respectively, both $\mathrm{p}>.10)$.

McCabe and Gotlib (1995) recommend determining whether reaction times to the stimuli are related to the emotion measures. The idea behind this is that if emotional state is affecting motivation to follow the task instructions, reaction times will be influenced by emotional state. However, median reaction time, averaged across neutral and sad stimuli, was uncorrelated with BDI-II and HAD-A scores $(\underline{r}=.041, \underline{n}=93$ and $\underline{r}=.040, \underline{n}=92$, both $\underline{p}>.10)$. This finding suggests that the effect of depression and anxiety on stimuli selection is not mediated by motivation.

As noted by Gotlib, McLachlan, and Katz (1988), a correlation between a measure of emotion and attention deployment does not, in itself, reveal which participants are showing a bias. To examine this issue, one sample t-tests were conducted comparing the mean percentage negative stimuli chosen by each 
group to chance (50\%), to determine which groups showed positive biases. As Figure 3 shows, nondepressed participants chose the negative stimuli significantly less frequently than $50 \%$ of the time $(\underline{\mathrm{M}}=47.88, \underline{\mathrm{SD}}=6.79, \underline{\mathrm{t}}[68]=$ 2.59, $\underline{\mathrm{p}}<.01)$, but dysphoric and dysphoric-depressed participants did not $(\underline{\mathrm{M}}=$ $50.5, \underline{\mathrm{SD}}=5.53, \underline{\mathrm{t}}[14]_{-}=.374, \underline{\mathrm{p}}>.10 ; \underline{\mathrm{M}}=49.33, \underline{\mathrm{SD}}=3.46, \underline{\mathrm{t}}[8]=2.59, \underline{\mathrm{p}}>$ .10). However, because the dysphoric and dysphoric-depressed groups were smaller in size than the nondepressed group, it could be argued that the reason these groups did not show a significant positive bias was because of lack of statistical power. For this reason, the data were reanalysed in groups of roughly equal size, by dividing the participants into quartiles by BDI-II score. The ranges of BDI-II scores within the quartiles were: lowest, 0 to $5(\underline{n}=57)$; medium-low, 6 to 9 ( $\underline{\mathrm{n}}=52)$; medium-high, 10 to $14(\underline{\mathrm{n}}=43)$; highest, 15 or over $(\underline{\mathrm{n}}=48)$.

As Figure 4 shows, participants in each of the two lower depression quartiles showed a significant positive bias $(\underline{\mathrm{M}}=47.59, \underline{\mathrm{SD}}=7.53$, $\underline{\mathrm{t}}[28]=1.73$; $\underline{\mathrm{M}}=47.15, \underline{\mathrm{SD}}=6.67, \underline{\mathrm{t}}[25]=2.18$, both $\underline{\mathrm{p}}<.05)$. Participants in each of the two upper depression quartiles did not show a significant bias $\underline{\underline{\mathrm{M}}}=50.10, \underline{\mathrm{SD}}=5.82$, $\underline{\mathrm{t}}[19]=0.08 ; \underline{\mathrm{M}}=49.89, \underline{\mathrm{SD}}=3.85, \underline{\mathrm{t}}[17]=0.122$, both $\underline{\mathrm{p}}>.10)$. As Figure 5 shows, only participants in the lowest HAD-A quartile showed a significant positive bias $(\underline{\mathrm{M}}=46.21, \underline{\mathrm{SD}}=7.55, \underline{\mathrm{t}}[27]=2.65, \underline{\mathrm{p}}<.01)$. Participants in the other three quartiles did not show a significant bias $\underline{\underline{M}}=48.86, \underline{\mathrm{SD}}=6.74, \underline{\mathrm{t}}[20]$ $=0.78 ; \underline{\mathrm{M}}=50.11, \underline{\mathrm{SD}}=5.83, \underline{\mathrm{t}}[18]=0.08 ; \underline{\mathrm{M}}=49.08, \underline{\mathrm{SD}}=4.33, \underline{\mathrm{t}}[23]=1.04$, all $\mathrm{p}>.10)$

T-tests were also conducted comparing mean percentage negative stimuli selected in the top and bottom quartiles of the data. This difference was near 
significance for depression $(\underline{\mathrm{t}}[43.76]=1.38, \underline{\mathrm{p}}<.10)$ and was significant for anxiety $(\underline{\mathrm{t}}[44.10]=1.71, \underline{\mathrm{p}}<.05)$.

Finally, to confirm that the mean differences in attention deployment were really the result of more participants in the lower quartiles showing positive biases, rather than fewer participants in these quartiles showing negative biases, post hoc analyses were conducted to determine the number of individuals in each quartile who were positively biased. By the binomial theorem, the chance of a given participant randomly selecting the negative stimuli $40 \%$ or less of the time (over 50 trials) is $\mathrm{p}=.10$. Table 2 shows the proportion of participants from each emotion group who were positively biased by this criterion. There are significantly more positively biased participants in the lowest depression group than in the highest depression group $(\underline{\mathrm{t}}[28]=2.70, \underline{\mathrm{p}}<.01)$, and also significantly more positively biased participants in the lowest anxiety group than in the highest anxiety group $(\underline{\mathrm{t}}[27]=2.71, \mathrm{p}<.01)$.

Table 2.

Attention Deployment Task: Proportion Positively Biased Participants in Each Emotion Group.

\begin{tabular}{|l|l|l|}
\hline & $\begin{array}{l}\text { Depression quartile } \\
\text { groups } \\
\text { Percentage positively } \\
\text { biased (at p } \leq .10)\end{array}$ & $\begin{array}{l}\text { Anxiety quartile groups } \\
\text { Percentage positively } \\
\text { biased (at } \mathrm{p} \leq .10)\end{array}$ \\
\hline Lowest & $21 \%$ & $21 \%$ \\
\hline Medium-low & $15 \%$ & $14 \%$ \\
\hline Medium-high & $5 \%$ & $11 \%$ \\
\hline Highest & $0 \%$ & $0 \%$ \\
\hline
\end{tabular}




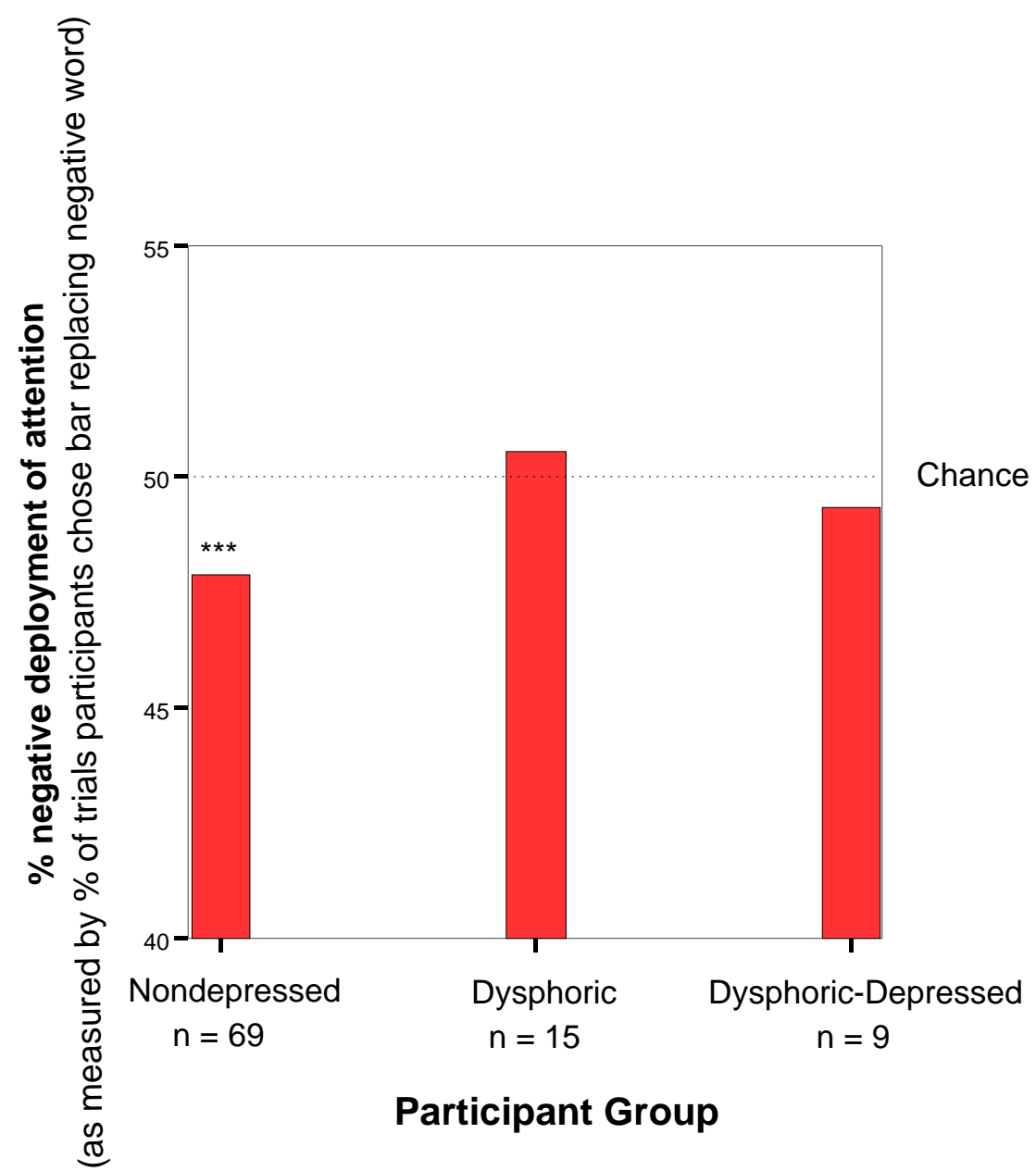

*** indicates significantly different from chance $(\mathrm{p}<.01)$.

Figure 3.

Relationship between depression category and percentage of trials attention deployed towards negative stimuli. 


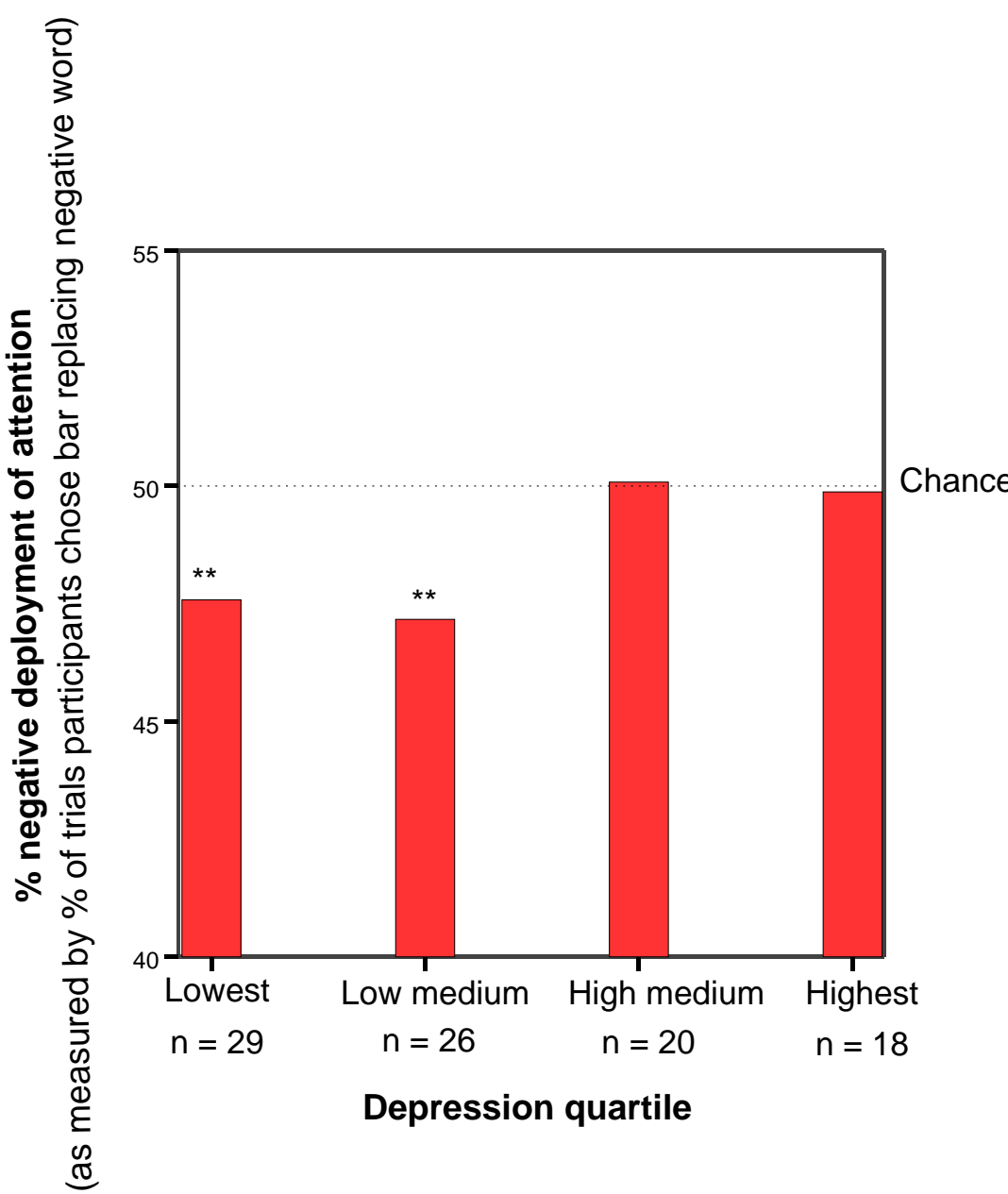

** indicates significantly different from chance $(\underline{\mathrm{p}}<.05)$.

\section{Figure 4.}

Relationship between depression quartile and percentage of trials attention deployed towards negative stimuli. 


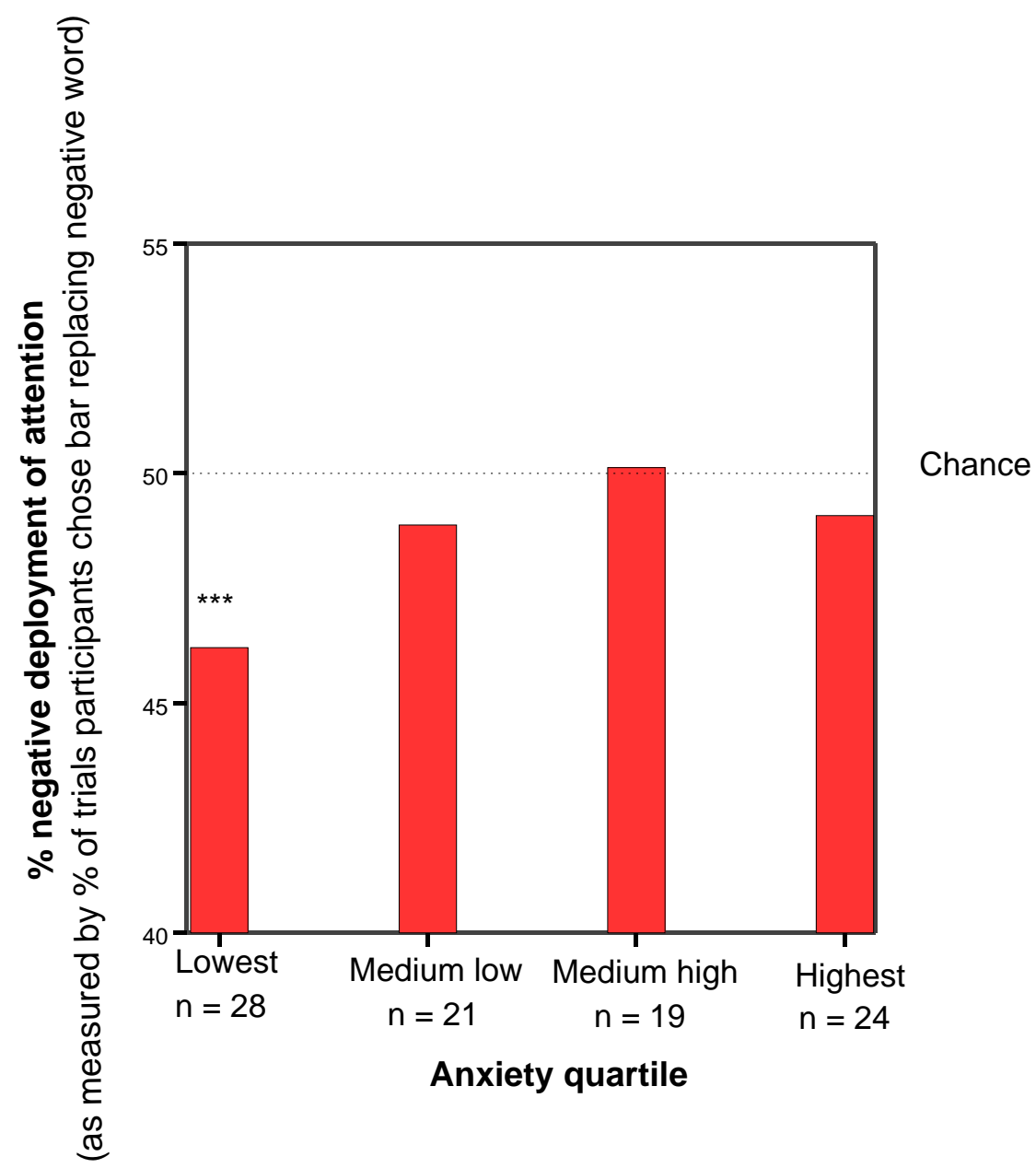

*** indicates significantly different from chance $(\mathrm{p}<.01)$.

Figure 5.

Relationship between anxiety quartile and percentage of trials attention deployed towards negative stimuli. 


\section{Discussion}

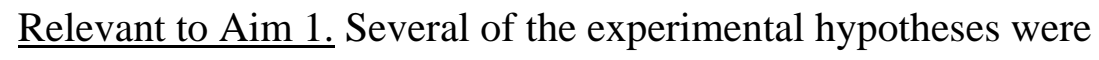
supported. As predicted, explicit memory bias was significantly associated with depression, but not with anxiety. In contrast, but also as predicted, perceptual implicit memory bias was significantly associated with anxiety, but not with depression. Strengthening this finding, the association between implicit memory bias and anxiety remained significant even when depression was controlled for. For the deployment of attention task, as predicted, participants low in depression and anxiety showed a positive bias. Also as predicted, both anxiety and depression were significantly related to the erosion of this positive bias. Partial correlations were unable to disentangle the contribution of anxiety from that of depression, but the trend was in the predicted direction.

Two of the hypotheses were not supported. It was predicted that conceptual implicit memory bias would be related to depression, but not to anxiety, and that emotional Stroop interference would be related to anxiety, but not to depression. However, neither of these tasks showed any relationship to either anxiety or depression. There are a number of possible explanations to consider in the case of the Stroop task. One possibility is that lack of statistical power led to the null finding, but although the emotional Stroop effect may be weak in nonclinical samples, and the effect has not always been consistently replicated (MacLeod \& Hagan, 1992, supraliminal condition), the sample size of this experiment was large in comparison to most existing studies. As discussed in the results section, this means that lack of statistical power was a relatively unlikely explanation for the lack of significant findings. Similarly, the accuracy 
of the computer's measurement of reaction time, and the number of trials, appear to have been adequate to accurately determine the mean reaction time in each condition for each participant. It is also unlikely that the use of key presses, rather than voice, to make responses is responsible for the nonreplication, because strong findings have been obtained using this modality before (Dalgleish, 1995). A final possibility is that the incorporation of the Stroop task into a battery of tasks involving the processing of negative information led to the null finding. Repeated exposure and habituation to the negative information may reduce the power of this information to distract the participant from the task at hand (Dozois \& Dobson, 2001). Lending strength to this idea, Dozois and Dobson (2001) also found no emotional Stroop effect occurred when they included the Stroop in a battery of other tasks with emotional content. Further research could test this possibility.

In the case of the conceptual implicit memory task, insufficient statistical power also seems an unlikely explanation, leaving two salient explanations. First, the free association task may be unreliable, as evidenced by P. C. Watkins et al.'s (2000) nonreplication of their earlier study. Second, because a clinically diagnosed sample was used in both the studies that have found an association between depression and conceptual implicit memory bias, it is possible that the effect occurs only in clinical populations. To test the first of these possibilities, Experiment 2 will investigate the relationship between depression and conceptual implicit memory bias using a different test of conceptual implicit memory.

Relevant to Aim 2. Support was obtained for the hypothesis that the somatic, rather than cognitive, symptoms of depression are related to information processing bias. In both cases where total depression score was related to 
negative bias, somatic factor score was significantly related to negative bias, but cognitive factor score was not. Moreover, when partial correlations were conducted involving the two factor scores and bias score, the somatic factor remained significantly associated with attentional bias, whereas the cognitive factor showed no evidence of influencing attention in a negative direction. Although nonsignificant, the pattern of partial correlations obtained for the explicit memory task was also consistent in direction with the hypothesis.

Relevant to Subsidiary Aim 1. Contrary to prediction, only participants in the bottom 25 to $50 \%$ of anxiety and depression scores showed a positive bias on the deployment of attention task. This contradicts the assumption of previous authors that subclinically depressed or anxious participants differ from the norm; rather, people who are unusually low in anxiety and depression appear to differ from the norm.

Relevant to Subsidiary Aim 4. Only two of the information processing tasks from Experiment 1 proved to be related to depression, and these relationships were moderate. Thus, the battery of tasks used in Experiment 1 would probably not, in itself, be useful as an assessment of depression. Experiment 2 examines a variety of other possible measures.

Rationale of Experiment 2. Experiment 1 found support for both the elaboration-priming hypothesis and the somatic-cognitive symptom hypothesis. Experiment 2 was designed to replicate these findings and extend them to a broader range of tasks. Thus, the experiment included a test of interpretation of ambiguous information - the homophone interpretation task (Eysenck et al., 1987). Williams et al. (1997) suggest that anxiety will control biases in homophone interpretation (p. 282). The logic behind this prediction is that 
priming makes material more likely to be activated when relevant perceptual features are presented. The homophone task involves presenting perceptual features of a negative word, and so priming, rather than elaboration, should affect homophone interpretation. Because Williams et al. (1997) argue that anxiety, rather than depression, affects priming, their theory predicts that anxiety, rather than depression, should affect homophone interpretation. Thus, including the homophone interpretation task in Experiment 2 means that the experiment can test a novel hypothesis derived from the theory.

Second, autobiographical memory is often thought to play a particularly important role in depression (Williams, 1992), and so a task that measures autobiographical memory was incorporated. The inclusion of an autobiographical memory task also has a fortuitous advantage. Experiments that have found that depressed people report unhappy autobiographical memories are often criticised on the grounds that any group differences shown could be due to veridical differences in life history, rather than to information processing bias. However, separating out the cognitive and somatic symptoms of depression provides a unique way of examining the relative contributions of cognitive bias and veridical differences. Negative life events should have the same impact on the two types of depression symptoms ${ }^{3}$. This means that the veridical difference explanation predicts similar correlations between rated happiness of recalled events and the somatic and cognitive symptoms of depression. Conversely, Experiment 1 showed that the somatic symptoms of depression were more strongly associated with negative biases in information processing than were the

\footnotetext{
${ }^{3}$ If anything, one would expect that events would affect the cognitive symptoms more; for example, failure experiences might lead to the belief that one had failed more than others, or a generally negative life history might lead to the belief that there is little hope for the future.
} 
cognitive symptoms. Thus, the information processing bias explanation predicts that the somatic symptoms of depression will be more strongly associated with the recall of unhappy memories than the cognitive symptoms will be. If this pattern is found, then this would support the hypothesis that the negative memories produced by depressed individuals are due to cognitive bias, rather than being solely due to veridical differences in life history.

The free association test of conceptual implicit memory bias did not appear to be affected by anxiety or depression in Experiment 1. As noted, P. C. Watkins et al. (2000) obtained similar results, but did find that a word-retrieval task, also intended as a test of bias in conceptual implicit memory, was related to depression. For this reason, in Experiment 2 the free association task was replaced with a word-retrieval task.

The explicit memory task was affected by depression in Experiment 1, but this effect may have occurred despite the presence of minor methodological problems. First, participants were not explicitly asked to encode the words selfreferentially, and it has been suggested that when information is self-referentially encoded, mood-congruent memory is especially likely to occur (Blaney, 1986). Second, the relatively small number of words involved in the task meant that a few participants showed perfect or near-perfect recall for either the positive or negative word list, meaning that ceiling effects may have occurred. Third, the word lists were not balanced for word frequency. Thus, in an attempt to obtain a larger effect size, the explicit memory task from Experiment 1 was adapted by presenting participants with a greater number of words to be learnt, by including neutral words in the task, and by balancing the word frequencies of the valenced lists. These objectives were accomplished by using the word list presented by 
Rusting (1999). The twin objectives of including an incidental memory task, and a memory task that explicitly required self-referent encoding, were accomplished by implementing incidental recall of the words encoded during the imagination task (the encoding stage of the implicit conceptual task).

Because autobiographical memory pleasantness was measured in the experiment, I took advantage of the data from the same task to measure autobiographical specificity. As noted in the literature review, overgeneral memory has been linked to clinical depression, but not to subclinical depression. It was hypothesised that this finding would be replicated. However, Merckelbach et al. (1996) did find that overgeneral recall was related to left-hemispheric thinking style. This finding was opposite in direction to their prediction that people with a right-hemispheric dominant thinking style would pay less attention to detail and would thus be more likely to produce overgeneral memories. These authors speculated that different findings might result if neutral, rather than emotional, cue words were used. Neutral cue words were used in Experiment 2, and for this reason, no prediction was ventured as to the direction of the hypothesised effect of thinking style on specificity. In addition to investigating the effect of thinking style, because different types of memory abilities are likely to be related (Jensen, 1998), it was predicted that poor memory for word lists would be associated with poor ability to recall specific autobiographical events.

It was also hypothesised that thinking style would influence performance on the emotional homophone task. Right hemispheric thinking is thought to be specialised for emotional processing (Borod et al., 2002), and so it was hypothesised that right-hemisphere dominant individuals would make a greater number of emotional interpretations of both positive and negative homophones. 
Because of time constraints on the length of the experiment, the emotional Stroop, perceptual implicit memory, and DOAT tasks were not included in Experiment 2.

In summary, it was hypothesised that

\section{$\underline{\text { Relevant to Aim } 1}$}

1. Negative bias in implicit conceptual, explicit incidental, and explicit intentional word list recall, and in autobiographical memory, would be directly associated with depression, but not directly associated with anxiety.

2. Negative bias in the emotional homophones task would be directly associated with anxiety, but not directly associated with depression. Specifically, based on the findings of Wenzlaff and Eisenberg (2001), it was expected that participants in negative emotional states would interpret neutral-negative homophones as negative, and neutral-positive homophones as neutral, more often than participants in positive mood states.

\section{$\underline{\text { Relevant to Aim } 2}$}

3. When negative bias scores correlated significantly with depression, they would correlate more strongly with the somatic factor than with the cognitive-affective factor of the inventory used.

\section{Relevant to Subsidiary Aim 2}

\section{Autobiographical specificity would not be related to depression or} anxiety, but would be related to thinking style as measured by the hemispheric preference test (PT; Zenhausern, 1978), and to memory ability on the explicit word list tasks. 
$\underline{\text { Relevant to Subsidiary Aim } 3}$

5. Right-hemispheric thinking style, as measured by the PT, would be associated with the production of greater numbers of emotional homophones, both positive and negative. 
Experiment 2

Method

\section{Overview}

Undergraduate psychology students, selected for high or low level of depressive symptomatology, completed five computer-administered tasks designed to measure mood-congruent bias. Four of these tasks examined memory. The tasks were a conceptual implicit memory test, an incidental selfreferent memory test, an intentional recall task, an autobiographical memory task, and a homophone interpretation task. Dependent variables were the number of negatively and positively valenced words recalled in each of the memory tasks, the number of specific memories recalled in the autobiographical memory task, the self-rated mean pleasantness of the autobiographical memories produced, and the number of negative and positive interpretations made of the aurally presented homophones. Following the computer-administered tasks, the participants were administered the BDI - II and the BAI.

\section{$\underline{\text { Participants }}$}

Participants were psychology undergraduate students at Victoria University, recruited from the psychology research pool. The preselection process involved 980 participants, and 148 participants took part in the 
experiment itself. Participants in the experiment had the option to supply demographic data. Of those who answered, 104 were female and 43 were male. The median age of the participants was 19 years. There were 123 New Zealand European participants, 11 Asian, 10 New Zealand Maori, and 3 of other ethnicity. Potential participants were excluded from the experiment if English was not their first language. Participants were given course credit for their participation.

\section{$\underline{\text { Materials }}$}

Participants were selected using the Beck Depression Inventory - Short Form (BDI-SF; Beck \& Beck, 1972), a 13-item questionnaire that correlates .96 with the original version of the Beck Depression Inventory (Beck \& Beamesderfer, 1974). After the experimental session, depression and anxiety were measured with the BDI-II and the Beck Anxiety Inventory (BAI; Beck \& Steer, 1993). The BDI-II was described in the method section of Experiment 1. The Beck Anxiety Inventory is empirically supported and widely recommended (Crits-Christoph \& Connolly, 1997), and there is evidence that it better discriminates anxiety from depression than does the other commonly used selfreport anxiety measure, the State-Trait Anxiety Inventory (Bieling et al., 1998).

Thinking style was measured using the Hemispheric Preference Test (PT; Zenhausern, 1978), a pencil-and-paper measure for which there is some evidence of validity (Merckelbach, de Jong, \& Muris, 1990; Merckelbach, Muris, Pool et al., 1996). Handedness was measured using a standard handedness questionnaire (Coren, 1992). 
$\underline{\text { Apparatus }}$

The experimental computer program was custom designed in Visual Basic, and is available on request. The program was run on Pentium III computers with standard colour CRT monitors, under Windows 98.

\section{Procedure}

Preselection of participants. Participants were administered the BDI-SF as part of a mass testing session. The questionnaire was accompanied by an assortment of questionnaires on other experimental topics, including the PT. This questionnaire served both to actually measure thinking style, and to distract experimental participants from their selection on the basis of mood. Participants with BDI-SF scores of zero to three, or of seven or above, were invited to take part in the experimental session described below, using a list of student identity numbers on a psychology research pool recruitment board. If participants asked why they had been chosen to take part in the study, they were informed that the decision was made on the basis of data collected during the mass testing session, but they were not informed that they had been chosen on the basis of their responses to the BDI-SF.

The experimental session. Experimental sessions began two weeks after the mass testing session. Participants were tested in groups of up to ten. Participants were seated in front of their own computer. The room was laid out such that individual responses were not visible to other participants. The experimenter was 
present throughout the duration of the experiment. Instructions on the individual tasks were displayed on the computer screen. Binary or Likert scale choices were made using the computer keyboard, and all other responses were made in a booklet supplied by the experimenter.

The introduction to the study, the encoding and retrieval stages of the implicit memory test, and the incidental recall task were conducted before the other tasks, in the order presented below. The remaining three experimental tasks were presented in counterbalanced order. It was not possible to counterbalance order of presentation of the implicit memory task, because this would have allowed the word stimuli used in the other experimental tasks to contaminate performance in the implicit memory task. Similarly, it was necessary for the incidental recall task to directly follow the implicit memory task, because it shared recall stimuli with that task.

After the participants had finished the computerised tasks, the computer administered an experimental purpose awareness question, and the experimenter administered the mood questionnaires.

Introduction to the study. The participants were told that they were taking part in an experiment looking at the effect of thinking style on a variety of tasks, and that the precise hypotheses of the experiment would be explained once it had been completed. They then gave their informed consent.

The experimenter then instructed the participants to fill out a questionnaire asking for basic demographic data, to wear the headphones supplied, to follow the instructions given by the computer with great care, and to ask the experimenter for help immediately if they should become confused by any aspect 
of the procedure. The questionnaire also asked for the participants’ student ID numbers, for matching with the BDI-SF scores collected earlier.

Handedness distracter questionnaire. The participants were then asked to fill out a handedness questionnaire (Coren, 1992), to reinforce the description of the study as an experiment on thinking styles.

The conceptual implicit memory test. In outline, the participants were asked to elaboratively encode a list of positive and negative words, and then their implicit memory for these words was tested with a word retrieval task. The procedure for this task was based on the conceptual implicit memory tasks used by Watkins, Vache, Verney, \& Mathews (1996) and Watkins, Martin, \& Stern (2000).

Encoding task. The encoding task was identical to the one used in Experiment 1, but this time an explanation was given for the task: participants were told that it measured aspects of their imaginative ability. The encoding task was followed by the thirty-second m-search distracter task also used in Experiment 1, and this was explained as a test of visual search ability. The participants were told that the task would be conducted in several short sessions to minimize visual fatigue.

Retrieval task. The participants were told that this task was a test of their linguistic ability. They were told that the computer would be present them with some definitions, and that they were to think of words that fitted those definitions. Each definition corresponded to a target word in either the studied or the unstudied word set, for a total of 32 definitions. In contrast to Watkins et al. (1996), who recorded responses on a tape recorder, in this experiment responses were written into the supplied booklet. The participants were given 20 seconds to 
respond to each cue definition, followed by a tone and the immediate onset of the next cue definition. Word definitions were adapted from a standard dictionary (Knight, 1993). Unlike in the experiment conducted by Watkins et al. (2000), participants were not supplied with the first letter of each target word. Positive and negative cue definitions were alternated.

The self-referent incidental recall task. Participants again completed the 30second distracter task. They were then explicitly asked to recall the 16 cue words presented in the imagination task. They were given two and a half minutes to complete this task, including reading the instructions. Responses were written into the supplied booklet.

The remaining three experimental tasks were conducted in counterbalanced order.

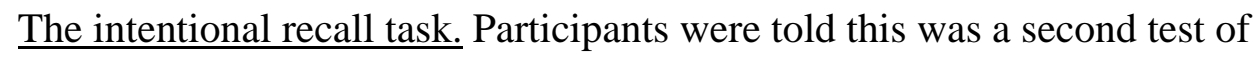
their memory ability. They were told that they would be given two minutes to learn a word list, and that they would have to recall it later in the experiment. They were then presented with the 36 words (12 negative, 12 positive, and 12 neutral) used in the incidental recall task conducted by Rusting (1999). All the words were presented simultaneously, positioned in individually randomised order with the restriction that no more than two words of any valence were presented in sequence. Following the learning procedure, the participants performed the m-search distracter task for one minute. They were then given two minutes to recall the words. Responses were written into the supplied booklet.

The autobiographical memory task. Participants were told that this was a test of their ability to recall events from their own lives. The computer directed participants to the appropriate page in their booklet, which contained the task 
instructions. Participants were told that they were to record the first specific memory that came to mind after they were presented with each of a series of twenty cue words. They were told that specific memories were of a single event, rather than a summary of a series of events, and that specific memories covered a time span of no more than one day. They were also given examples of three specific and three nonspecific memories. They were then told that they would have a maximum of 50 seconds to recall and record each event, after which they would be prompted to stop trying and move on to the next cue word ${ }^{4}$. They were told to hit the space bar to indicate when they had remembered and recorded an event description. The instruction sheet contained two example cues, to which they were to produce appropriate responses and show these to the experimenter to confirm they had understood the task instructions regarding specificity. The experimenter gave the participants further opportunity to practice if they did not produce at least one specific example. If only one specific example was produced, the experimenter explained this and explained why. The computer then presented ten neutral cue words to the participants in the manner described above. The cue words were replicated from List A of Clark (1982), and presented in fixed order. Participants were then asked three questions about each of the events they had described:

1. How vividly do you remember the event now?

2. How pleasant was the event?

3. How sure are you that the event really happened?

The questions were answered on anchored nine-point Likert scales. Question two was the only critical item; the other questions were used to distract the

\footnotetext{
${ }^{4}$ There were two reasons for using time pressure in this task. First, I wanted to move participants through the tasks as quickly as possible. Second, introducing time pressure helps distract participants from the true nature of the task (Kumari et al., 1998).
} 
participants from the real purpose of the experiment. The participants were asked whether the events were pleasant or unpleasant rather than happy or unhappy because the former question is less suggestive of the experimental purpose, but produces answers very highly correlated with answers to the latter question (Mayo, 1983). Similarly, self-ratings rather than independent ratings of pleasantness were conducted, because self- and independent ratings of pleasantness are very highly correlated (Mayo, 1983; Teasdale \& Fogarty, 1979). Participants were instructed to move rapidly through the questions, and told that it was their first impressions that were of most interest. Responses were given using the computer keyboard.

The homophone interpretation task. Participants were told that this was a test of spelling ability, and that spelling ability is related to general memory performance. The instructions explained that words would be presented through the headphones, and that the participants were to spell the words in the booklet provided. They were also informed that they would be under time pressure during the experiment, and so to just guess when they were unsure. The participants were then presented with five neutral practice words, and given the opportunity to practice again if necessary. They were then presented with the experimental word list. The critical items were 12 negative and 12 positive homophones. Twenty-one of these were sourced from Wenzlaff \& Eisenberg (2001), but the other three words used in this experiment were replaced because of local differences in pronunciation and word meaning - these words were sourced from Rusting (1999) and Halberstadt, Niedenthal, and Kushner (1995). There was no significant difference in mean written word frequency (Kucera \& Francis, 1967) between the emotional and neutral interpretations of either the 
positive or negative homophones (both $\mathrm{p}>.10$ ). There were also 36 unvalenced filler words, including homophones and non-homophones. Most of these were sourced from a list of words that had been misspelt in university entrance exam papers (Wing \& Baddeley, 1980). Following Wenzlaff and Eisenberg (2001), the words were presented with a three second interval between the onsets of sequential words.

Administration of the mood questionnaires. After the participants had finished all of the computerized tasks, the computer prompted them to turn to the final page of the experimental booklet, which contained two ruled lines and was simply labelled “Comprehension”. They were instructed "Please very briefly describe what you think the intended purpose of this experiment was, or else write 'Don't know'”. The computer then prompted the participants to ask the experimenter for the final questionnaires. The experimenter gave the participants the BDI-II, the BAI, and a brief questionnaire asking whether they had a history of treatment for anxiety or depression. Participants were asked to record their randomised matching codes in the place of the field labelled "name”. Participants were given the option of not answering some or all questionnaire items. Participants were then thoroughly debriefed, and asked not to talk to other students about the purpose of the experiment.

A word about word stimuli. Except for the sharing of target words between the implicit memory and incidental recall tasks, no word stimulus was used in more than one experimental task. 


\section{Results}

The experimenter was blind to responses to the emotion measures while scoring task responses, and while making decisions about excluding participants from analyses. Exclusion criteria were determined before the data were analysed. One-tailed t-tests were used for the analyses of mood and bias (see discussion of the use of this statistical method in the results section of Experiment 1), but twotailed t-tests were used in the analyses of the additional hypotheses, because the predictions made were based on theory rather than replication of existing experiments. All power calculations were conducted using the GPOWER computer program (Erdfelder et al., 1996).

\section{Cleaning of the Data}

\section{$\underline{\text { Exclusions for Poor Data Quality }}$}

Participants were excluded from the homophones task if, more than once, they recorded both possible spellings of a homophone (which may indicate too much knowledge of the experimental purpose). Participants were also excluded for failing to give responses to more than two critical items. Ten participants were excluded from the homophone task for these reasons. Participants were excluded from the incidental memory task for producing a high outlier number of intrusions (nine or more; four participants excluded), which was interpreted as failure to understand task instructions. One participant was excluded from the autobiographical memory valence task, because all except one of the ratings of 
vividness, pleasantness, and certainty were invariant. Participants were also excluded from analyses involving individual tasks in the rare instance that they failed to correctly complete that task, and from the autobiographical memory specificity analyses if they failed to present examples to the experimenter to be checked (three participants excluded).

\section{Excluding Participants Because of Understanding of the Experimental Purpose}

Participants whose answers to the comprehension question demonstrated that they understood the overall experimental purpose were excluded from nearly all analyses, because of their susceptibility to demand effects. They were, however included in the autobiographical memory specificity analyses, because no participant evidenced understanding of this aspect of the task. Eleven participants were excluded because of experimental understanding, nine from the nondepressed group, one from the dysphoric group, and one from the dysphoricdepressed group (see below for the division of participants into depression group). Similarly, three of these participants fell into the low anxiety group, four into the medium-low anxiety group, three into the medium-high anxiety group, and one into the high anxiety group.

Participants who appeared to understand the mechanism of individual tasks, for example, that the linguistic ability test was an implicit memory test or that the spelling task involved choice between spellings of homophones, were excluded from analyses of these specific tasks. For this reason, an additional ten participants were excluded from the homophone task, five from the linguistic ability task, and six from the autobiographical memory task. 


\section{Depression and Anxiety Measures}

The mean BDI-II score was 9.99, which is similar to the mean value of 9.11 found in the large unselected student sample reported by Dozois, Dobson, and Ahnberg (1998). The standard deviation of the BDI-II scores (9.63) was somewhat higher than the standard deviation (7.57) found in that sample, as would be expected given that the participants were selected for high or low depression scores. The mean BAI score was $9.71(\underline{\mathrm{SD}}=8.12)$, which is similar to the mean (9.15) and standard deviation (7.41) found for a large unselected sample by Lovibond and Lovibond (1995).

\section{Division of Participants into Groups}

As in Experiment 1, participants were divided into nondepressed $(\underline{\mathrm{n}}=$ 104), dysphoric $(\underline{\mathrm{n}}=20)$ or dysphoric-depressed groups $(\underline{\mathrm{n}}=24)$, as recommended by Dozois et al. (1998). By participant report, 5\% of nondepressed participants, 25\% of dysphoric participants, and 29\% of dysphoric-depressed participants had undergone formal treatment for depression during the past year; this supports the contention that the levels of distress examined by this study were in at least some cases comparable to those encountered in clinical practice. Also as in Experiment 1, participants were divided into anxiety groups according to BAI quartile - low anxiety (BAI scores of 0 to $3, \underline{n}=36$ ), medium-low anxiety (scores of 4 to $8, \underline{n}=40$ ), medium-high anxiety (scores of 9 to 13 , $\underline{n}=35$ ), or high anxiety (scores of 14 or above, $\underline{\mathrm{n}}=36$ ). By participant report, $0 \%, 10 \%, 6 \%$ 
and $19 \%$ of participants in each quartile (respectively) had undergone formal treatment for anxiety during the past year.

\section{$\underline{\text { Word Retrieval Task }}$}

The dependent variables in the word retrieval task were calculated in an identical way to those in the word stem completion and word association tasks of Experiment 1. As Table 3 shows, neither adjusted for baseline positive nor adjusted for baseline negative target word production was related to either BDI-II or BAI score. This means that no mood-congruency effects were shown. For the participants as a whole, adjusted for baseline primed number of target words produced was significantly greater than baseline for both the positive stimuli $(\underline{\mathrm{M}}$ $=1.23, \underline{\mathrm{SD}}=1.59$, vs. $\underline{\mathrm{M}}=-0.03, \underline{\mathrm{SD}}=1.24 ; \underline{\mathrm{t}}[130]=8.29, \underline{\mathrm{p}}<.001)$ and the negative stimuli $(\underline{\mathrm{M}}=1.27, \underline{\mathrm{SD}}=1.65$, vs. $\underline{\mathrm{M}}=-0.02, \underline{\mathrm{SD}}=1.39 ; \underline{\mathrm{t}}[130]=8.41$, $\mathrm{p}$ $<.001)$. This shows that priming occurred. 


\section{Emotion-Congruent Information Processing 105}

Table 3.

Correlations Between Information Processing Bias Scores and Emotion Measures.

\begin{tabular}{|c|c|c|c|c|c|c|c|c|}
\hline Task & Explicit memory & Incidental memory & $\begin{array}{l}\text { Conceptual } \\
\text { implicit memory } \\
\text { (word retrieval) }\end{array}$ & & $\begin{array}{l}\text { Interpretation of } \\
\text { ambiguity } \\
\text { (Homophone task) }\end{array}$ & & $\begin{array}{l}\text { Autobio- } \\
\text { graphical } \\
\text { pleasantness }\end{array}$ & $\begin{array}{l}\text { Autobio- } \\
\text { graphical } \\
\text { specificity }\end{array}$ \\
\hline Emotion measure & $\begin{array}{l}\text { Number of } \\
\text { negative words } \\
\text { recalled (positive } \\
\text { words recalled as } \\
\text { covariate) }\end{array}$ & $\begin{array}{l}\text { Number of negative } \\
\text { words recalled } \\
\text { (other words } \\
\text { recalled as } \\
\text { covariate) }\end{array}$ & $\begin{array}{l}\text { Primed positive } \\
\text { target words } \\
\text { produced } \\
\text { (adjusted for } \\
\text { baseline) }\end{array}$ & $\begin{array}{l}\text { Primed negative } \\
\text { target words } \\
\text { produced (adjusted } \\
\text { for baseline) }\end{array}$ & $\begin{array}{l}\text { Number of negative } \\
\text { interpretations } \\
\text { made }\end{array}$ & $\begin{array}{l}\text { Number of } \\
\text { positive } \\
\text { interpretations } \\
\text { made }\end{array}$ & $\begin{array}{l}\text { Mean rated } \\
\text { pleasantness } \\
\text { of events }\end{array}$ & $\begin{array}{l}\text { Number of } \\
\text { specific events } \\
\text { produced }\end{array}$ \\
\hline BDI-II & $\begin{array}{l}\underline{\mathrm{pr}}=-.015 \\
\underline{\mathrm{df}}=133\end{array}$ & $\begin{array}{l}\mathrm{pr}=.037 \\
\mathrm{p}=.336 \\
\underline{\mathrm{df}}=128\end{array}$ & $\begin{array}{l}\underline{\mathrm{r}}=.028 \\
\underline{\mathrm{n}}=131\end{array}$ & $\begin{array}{l}\underline{\mathrm{r}}=-.021 \\
\underline{\mathrm{n}}=131\end{array}$ & $\begin{array}{l}\underline{\mathrm{r}}=.167 * * \\
\underline{\mathrm{p}}=.041 \\
\underline{\mathrm{n}}=110\end{array}$ & $\begin{array}{l}\underline{\mathrm{r}}=.094 \\
\underline{\mathrm{n}}=110\end{array}$ & $\begin{array}{l}\underline{\mathrm{r}}=-.145 * * \\
\mathrm{p}=.050 \\
\underline{\mathrm{n}}=130\end{array}$ & $\begin{array}{l}\underline{\mathrm{r}}=.075 \\
\underline{\mathrm{n}}=145\end{array}$ \\
\hline BDI-II somatic factor & & & & & $\begin{array}{l}\underline{r}=.144 * \\
\underline{p}=.067 \\
\underline{n}=110\end{array}$ & & $\begin{array}{l}\underline{\mathrm{r}}=-.164 * * \\
\mathrm{p}=.031 \\
\underline{\mathrm{n}}=130\end{array}$ & \\
\hline $\begin{array}{l}\text { BDI-II cognitive- } \\
\text { affective factor }\end{array}$ & & & & & $\begin{array}{l}\underline{\mathrm{r}}=.176 * * \\
\underline{\mathrm{p}}=.033 \\
\underline{\mathrm{n}}=110\end{array}$ & & $\begin{array}{l}\underline{\mathrm{r}}=-.108 \\
\underline{\mathrm{p}}=.110 \\
\underline{\mathrm{n}}=130\end{array}$ & \\
\hline BAI & $\begin{array}{l}\mathrm{pr}=-.102 \\
\underline{\mathrm{df}}=133\end{array}$ & $\begin{array}{l}\underline{\mathrm{pr}}=-.001 \\
\underline{\mathrm{df}}=128\end{array}$ & $\begin{array}{l}\underline{\mathrm{r}}=.087 \\
\underline{\mathrm{n}}=130\end{array}$ & $\begin{array}{l}\underline{\mathrm{r}}=.006 \\
\mathrm{p}=.475 \\
\underline{\mathrm{n}}=130\end{array}$ & $\begin{array}{l}\underline{\mathrm{r}}=.188 * * \\
\mathrm{p}=.025 \\
\underline{\mathrm{n}}=109\end{array}$ & $\begin{array}{l}\underline{\mathrm{r}}=.116 \\
\underline{\mathrm{n}}=109\end{array}$ & $\begin{array}{l}\underline{\mathrm{r}}=-.098 \\
\mathrm{p}=.134 \\
\underline{\mathrm{n}}=129\end{array}$ & $\begin{array}{l}\underline{\mathrm{r}}=.113 \\
\underline{\mathrm{n}}=144\end{array}$ \\
\hline $\begin{array}{l}\text { Partial correlation } \\
\text { with BDI-II } \\
\text { controlling for BAI }\end{array}$ & & & & & $\begin{array}{l}\underline{\mathrm{pr}}=.045 \\
\mathrm{p}=.320 \\
\underline{\mathrm{df}}=106\end{array}$ & & $\begin{array}{l}\underline{\mathrm{pr}}=-.107 \\
\underline{\mathrm{p}}=.114 \\
\underline{\mathrm{df}}=126\end{array}$ & \\
\hline $\begin{array}{l}\text { Partial correlation } \\
\text { with BAI controlling } \\
\text { for BDI-II }\end{array}$ & & & & & $\begin{array}{l}\mathrm{pr}=.097 \\
\mathrm{p}=.160 \\
\underline{\mathrm{df}}=106\end{array}$ & & $\begin{array}{l}\mathrm{pr}=.009 \\
\underline{\mathrm{df}}=126\end{array}$ & \\
\hline
\end{tabular}

* indicates near significance $(\mathrm{p}<.10) . * *$ indicates significant at $\mathrm{p}<.05 . * * *$ indicates significant at $\mathrm{p}<.01$. **** indicates significant at $\mathrm{p}<.001$. All $\underline{\mathrm{t}}$-tests were onetailed. $\mathrm{p}$ values for correlations in the direction opposite to that predicted are not reported as one-tailed t-tests have no statistical power in this circumstance.The use of two-tailed t-tests would not have produced any additional significant findings. BDI-II factor correlations were calculated only when the BDI-II correlated significantly with the relevant variable. Partial correlations were calculated only when the BDI-II or BAI correlated significantly with the relevant variable. 


\section{Incidental Recall Task}

As for the analysis of the explicit memory task in Experiment 1, the dependent variable was the number of negative words recalled, but the number of positive words recalled was included as a covariate in the analysis to control for general memory ability. As Table 3 shows, memory bias was not significantly correlated with either depression or anxiety. Including counterbalancing condition as a covariate (to control for the different word lists the participants were asked to recall) does not change the pattern of results.

\section{Intentional Recall Task}

As for the analysis of the explicit memory task of Experiment 1, the dependent variable was number of negative words recalled. Number of other words recalled (positive plus neutral) was included as a covariate to control for general memory ability. As Table 3 shows, memory bias was not significantly correlated with either depression or anxiety.

\section{$\underline{\text { Post-hoc Power Calculations for the Wordlist Memory Tasks }}$}

A post-hoc power analysis was conducted to gain an idea of whether the failure of the experiment to find significant associations between BDI-II score, BAI score, explicit memory bias, and implicit priming was due to insufficient statistical power. If the true $\underline{\mathrm{r}}$ between bias score and BDI-II or BAI score was .15 , then to obtain $90 \%$ power with 128 participants in a one-tailed correlational 
t-test would require setting alpha to .33 . As Table 3 shows, none of the observed correlations were significant by this criterion. Although the post-hoc nature of these calculations calls for caution, the results indicate that insufficient power is a relatively unlikely explanation of the lack of significant relationships between the variables.

\section{Homophone Interpretation Task}

The dependent variables were the number of positive and negative homophone interpretations made by the participants. As Table 3 shows, the predicted correlation between anxiety score and negative interpretations was significant $(\underline{\mathrm{r}}=.188, \underline{\mathrm{p}}<.05)$, as was the correlation between depression score and negative interpretations $(\underline{\mathrm{r}}=.167, \underline{\mathrm{p}}<.05)$. The partial correlation between depression and negative interpretations, controlling for anxiety, was nonsignificant $(\mathrm{pr}=.045, \underline{\mathrm{p}}>.10)$. The partial correlation between anxiety and negative interpretations, controlling for depression, was also nonsignificant ( $\mathrm{pr}=$ $.097, \underline{\mathrm{p}}>.10)$.

The relationships of the somatic and cognitive factors of depression to negative homophone production were also compared. The correlation between the somatic factor of depression and negative interpretations was near significance $(\underline{r}=.144, \underline{p}<.10)$, and the correlation between the cognitiveaffective factor of depression and negative interpretations was significant $(\underline{\mathrm{r}}=$ $.176, \mathrm{p}<.05)$. The partial correlation of negative interpretations with somatic depression, controlling for cognitive depression, was nonsignificant ( $\underline{p r}=-.001$, $\underline{p}>$.10). Similarly, the partial correlation of negative interpretations with 
cognitive depression, controlling for somatic depression, was nonsignificant (pr $=.102, \underline{\mathrm{p}}>.10)$.

The correlation between depression and positive homophone interpretations made was nonsigificant $(\underline{\mathrm{r}}=.094, \underline{\mathrm{p}}>.10)$, as was the correlation between anxiety and positive interpretations $(\underline{r}=.116, \underline{p}>.10)$. Post-hoc power calculations showed that the lack of significant results was unlikely to be due to insufficient power. If the true $\underline{r}$ between bias score and BDI-II or BAI score was .15 , then to obtain $90 \%$ power with 109 participants in a one-tailed correlational t-test would require setting alpha to .38 . As Table 3 shows, because both of the observed correlations were in the opposite direction to that predicted, neither were significant by this criterion. Although the post-hoc nature of these calculations calls for caution, the results indicate that insufficient power is a relatively unlikely explanation of the lack of significant relationships between the variables.

The correlations reported are qualitatively similar when all participants who completed the tasks were included in the analyses, including those who showed understanding of the experiment in the catch question, or were excluded from the main analyses because of poor data quality. This shows that the results are not an artifact of the exclusion criteria used.

The mean number of negative homophone interpretations made by the different groups of participants are illustrated in Figures 6 and 7. The differences between the top and bottom groups are near significance for both depression ( $\underline{\mathrm{M}}$ $=6.47, \underline{\mathrm{SD}}=1.43$, vs. $\underline{\mathrm{M}}=7.00, \underline{\mathrm{SD}}=1.61, \underline{\mathrm{t}}[93]=1.39, \underline{\mathrm{p}}<.10)$ and anxiety $(\underline{\mathrm{M}}$ $=6.56, \underline{\mathrm{SD}}=1.50$, vs. $\underline{\mathrm{M}}=7.11, \underline{\mathrm{SD}}=1.20, \underline{\mathrm{t}}[51]=1.47, \underline{\mathrm{p}}<.10)$. 


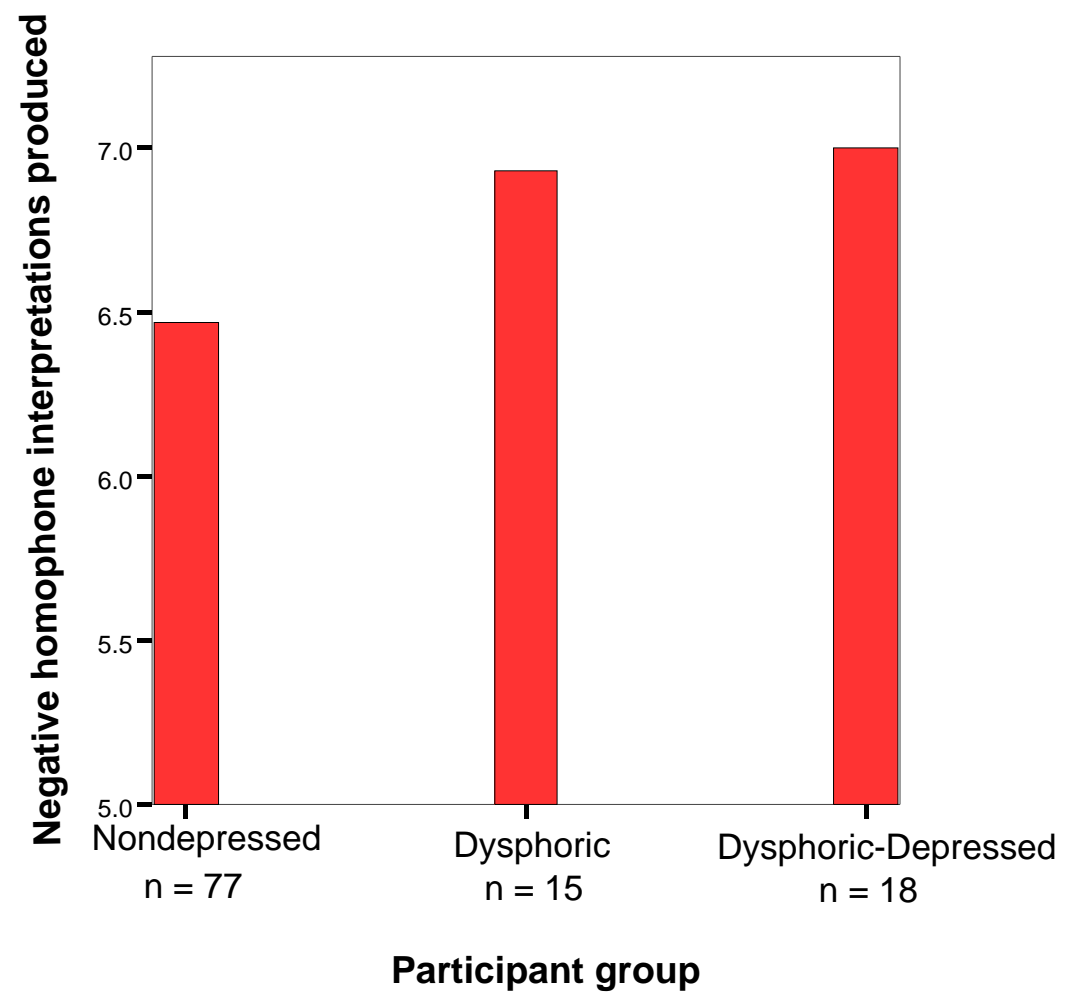

Figure 6.

Relationship between depression category and number of negative homophone interpretations made. 


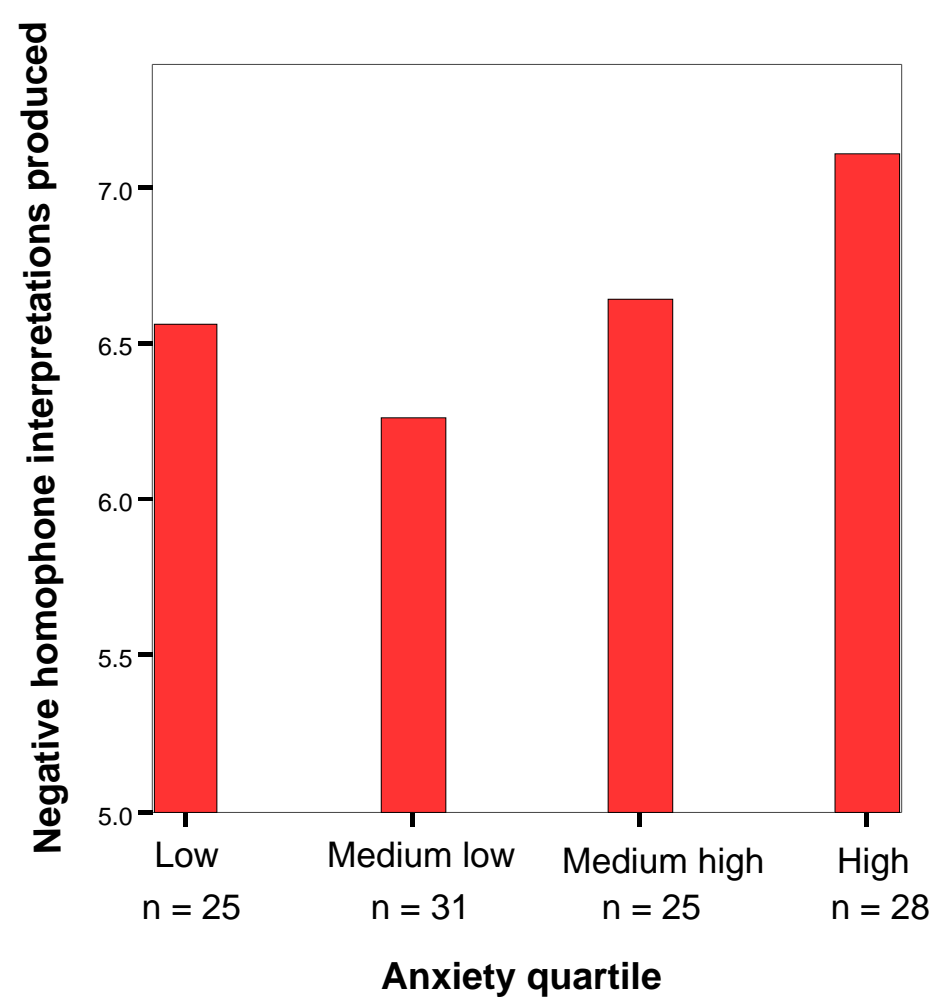

Figure 7.

$\underline{\text { Relationship between anxiety quartile and number of negative homophone }}$ interpretations made. 


\section{Autobiographical Recall Valence Task}

The dependent variable was the mean self-rated pleasantness of the autobiographical events reported by the participants. As Table 3 shows, the predicted correlation between depression and autobiographical pleasantness was significant $(\underline{r}=-.145, \underline{p}=.05)$, but the correlation between anxiety and autobiographical pleasantness was nonsignificant $(\underline{\mathrm{r}}=-.098, \underline{\mathrm{p}}>.10)$. The partial correlation between depression and pleasantness, controlling for anxiety, was not significant ( $\mathrm{pr}=-.107, \mathrm{p}>.10)$. The partial correlation between anxiety and pleasantness, controlling for depression, was also nonsignificant $(\mathrm{pr}=.009, \mathrm{p}>$ $.10)$.

The relationships of the somatic and cognitive factors of depression to autobiographical pleasantness were also compared. The correlation between the somatic factor of depression and negative interpretations was significant $(\underline{r}=-$ $.164, \mathrm{p}<.05$ ), but the correlation between the cognitive-affective factor of depression and pleasantness was nonsignificant $(\underline{r}=-.108, \underline{p}>.10)$. The partial correlation of pleasantness with somatic depression, controlling for cognitive depression, was near significance $(\mathrm{pr}=-.131, \underline{\mathrm{p}}<.10)$. The partial correlation of pleasantness with cognitive depression, controlling for somatic depression, was nonsignificant $(\mathrm{pr}=.043, \mathrm{p}>.10)$.

Again, the results for this task are qualitatively similar when all participant data is included, regardless of understanding of the experimental purpose or exclusion from the main analyses for lack of data quality. This shows that the results are not an artifact of the exclusion criteria used. 
The mean self-rated autobiographical pleasantness of the events reported by the different depression groups is illustrated in Figure 8 . The difference between the nondepressed and depressed-dysphoric group neared significance $(\underline{\mathrm{M}}=5.60, \underline{\mathrm{SD}}=1.22$, vs. $\underline{\mathrm{M}}=5.23, \underline{\mathrm{SD}}=0.96, \underline{\mathrm{t}}[109]=1.27, \underline{\mathrm{p}}=.10)$. 


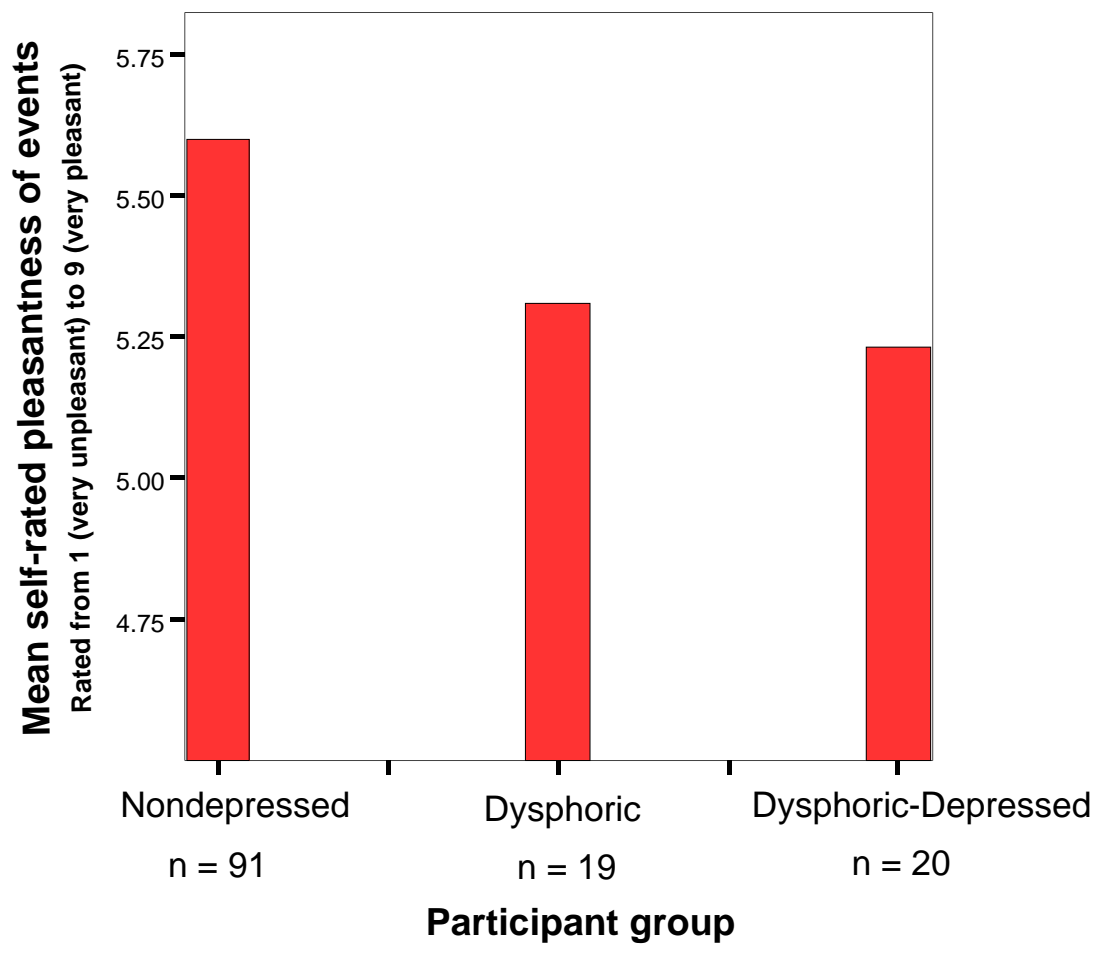

Figure 8.

$\underline{\text { Relationship between depression category and self-rated pleasantness of }}$ autobiographical events recalled in response to neutral cues. 


\section{Autobiographical Recall Specificity}

The dependent variable was the number of specific memories produced by the participants, as rated by the experimenter. Past research has shown that independent raters can reliably discriminate specific memories from nonspecific memories (Williams \& Dritschel, 1988). As shown in Table 3, number of specific memories produced was not significantly related to depression $(\underline{r}=.075$, $\underline{\mathrm{p}}>.10)$ or anxiety $(\underline{\mathrm{r}}=.113, \underline{\mathrm{p}}>.10)$. Post-hoc power calculations showed that the lack of significant results was unlikely to be due to insufficient power. If the true $\underline{r}$ between bias score and BDI-II or BAI score was .15, then to obtain $90 \%$ power with 145 participants in a one-tailed correlational t-test would require setting alpha to .29. As Table 3 shows, because both of the observed correlations were in the opposite direction to that predicted, neither were significant by this criterion. Although the post-hoc nature of these calculations calls for caution, the results indicate that insufficient power is a relatively unlikely explanation of the lack of significant relationships between the variables.

Because past authors have argued that overgeneral recall is related only to clinical levels of distress, those participants who reported they had undergone psychotherapeutic or pharamacological treatment for depression within the last year were selected for analysis. This group $(\underline{\mathrm{n}}=17)$ was compared to the other participants $(\underline{\mathrm{n}}=126)$. There was no significant between-group difference in number of specific memories produced $(\underline{\mathrm{M}}=9.15, \underline{\mathrm{SD}}=1.26$, vs. $\underline{\mathrm{M}}=9.12$, $\underline{\mathrm{SD}}$ $=1.22, \underline{\mathrm{t}}[143]=.10, \mathrm{p}>.10)$. 


\section{Additional Planned Analyses}

Because all predictions being tested by the comparisons in this section were based on theory rather than informed by prior research findings, two-tailed rather than one-tailed t-tests were conducted.

Hemispheric Preference Test (PT) Score and Autobiographical Recall Specificity

Higher scores on the PT indicate greater reliance on left-hemisphere thinking style. It was predicted that hemisphericity would relate to specificity of autobiographical memory, but no prediction was made as to the direction of this relationship. Left-hemispheric thinking style was related to greater specificity of recalled events $(\underline{r}=.253, \underline{p}<.01)$. The cases where participants produced nonspecific memories were broken down into type - categorical memories, extended memories, non-event memories, and failures to answer (Williams \& Dritschel, 1992). Left-hemispheric thinking was related to the production of fewer categorical memories $(\underline{r}=-.316, \underline{p}<.001)$, but was not significantly related to production of extended memories $(\underline{r}=-.110, \underline{p}>.10)$ or memories that were not of events $(\underline{r}=-.089, \underline{p}>.10)$. Contrary to the pattern for the other types of nonspecific memories, there was a near significant trend for left-hemispheric thinking to predict more answers left blank $(\underline{r}=.138, \underline{p}=.10)$. 
Number of Words Recalled in the Explicit Memory Tasks and Autobiographical $\underline{\text { Recall Specificity }}$

It was hypothesized that overgeneral autobiographical memory would be associated with poor memory in general. This hypothesis was supported. Number of specific memories produced was significantly correlated with number of words recalled, totalled across the two explicit memory tasks $(\underline{r}=.195, \underline{n}=130$, $\mathrm{p}<.05)$. Because thinking style was also associated with specificity of memory, this analysis was repeated using a partial correlation, controlling for thinking style. The resulting correlation was significant $(\underline{\mathrm{pr}}=.217, \underline{\mathrm{df}}=123, \underline{\mathrm{p}}<.05)$. Thinking style, controlling for total number of words recalled, was also significantly associated with number of specific words produced $(\underline{\mathrm{pr}}=.316$, $\underline{\mathrm{df}}=$ 123, $\mathrm{p}<.001)$. Thus, better wordlist memory ability and left-hemispheric thinking style were independently related to greater autobiographical memory specificity.

$\underline{\text { Hemispheric Preference Test (PT) Score and Homophone Interpretation }}$

Higher scores on the PT indicate greater reliance on left-hemisphere thinking style. It was predicted that left-hemisphere dominant individuals would produce fewer emotion-related homophones than right-hemisphere dominant individuals, for both positive and negative stimuli. The hypothesis was supported: PT score was significantly correlated with number of negative homophones produced $(\underline{\mathrm{r}}=-.195, \underline{\mathrm{p}}<.05, \underline{\mathrm{n}}=106)$. It was near significantly 
correlated with number of positive homophones produced $(\underline{r}=-.188, \underline{p}<.06, \underline{n}=$ 106), and it was significantly correlated with total number of emotional (positive plus negative) homophones produced $(\underline{\mathrm{r}}=-.256, \underline{\mathrm{p}}<.01, \underline{\mathrm{n}}=106)$.

\section{$\underline{\text { Additional Analyses }}$}

\section{Autobiographical Recall Specificity and Autobiographical Pleasantness}

One final association was noted that was not the result of a planned comparison. For this reason, it should be interpreted with caution, but it is reported because of it's strength and possible importance. There was a correlation between number of specific memories produced and self-rated mean pleasantness of autobiographical events $(\underline{\mathrm{r}}=-.251, \underline{\mathrm{n}}=127, \underline{\mathrm{p}}<.01)$. Breaking this down to look at the different kinds of non-specific memories, both categorical $(\underline{\mathrm{r}}=.248, \underline{\mathrm{n}}=127, \underline{\mathrm{p}}<.01)$ and non-event memories $(\underline{\mathrm{r}}=.297, \underline{\mathrm{n}}=$ $127, \underline{p}=.001$ ) were significantly and positively associated with mean pleasantness rating. Number of extended memories produced was not significantly associated with mean pleasantness $(\underline{\mathrm{r}}=.029, \underline{\mathrm{n}}=127, \underline{\mathrm{p}}>.10)$, while number of non-responses was significantly negatively correlated with mean pleasantness $(\underline{\mathrm{r}}=-.185, \underline{\mathrm{n}}=127, \underline{\mathrm{p}}<.05)$. In summary, producing categorical and non-event memories is associated with the recall of pleasant events, while not recalling any event at all is associated with the recall of unpleasant events. 
Summary of the Findings of Experiments 1 and 2

Relevant to Aim 1. The first main aim of this thesis was to directly compare the information processing correlates of anxiety and depression. Table 4 summarises the overall pattern of results for those tasks where negative mood was related to information processing bias. The theory that Williams et al. (1997) put forward to explain the relationship between information processing biases and anxiety and depression predicts that the two tasks to the left of the grey line, wordlist and explicit memory, will be more closely related to depression than to anxiety, because these tasks rely on elaboration rather than priming. Williams et al.'s (1997) theory also predicts that the three tasks to the right of the grey line, deployment of attention, perceptual implicit memory, and homophone interpretation, will be more closely related to anxiety than to depression, because these tasks rely on priming rather than elaboration.

Were these predictions supported? There are two ways of examining the hypothesis. The first is to simply look at whether anxiety or depression correlate significantly with bias score. This is the method that is in effect used when existing reviewers compare the results of studies that looked only at anxiety and bias to the results of studies that looked only at depression and bias, although the present studies have the methodological advantage of eliminating methodological differences between studies. Table 4 shows that for both tasks where the model predicts that bias will relate to depression, this prediction was supported by a significant correlation. In neither case was there a significant association between anxiety and bias. Likewise, for the three tasks where the model predicts that bias 
would relate to anxiety, these predictions were supported by significant correlations. However, in two of these cases there were also significant correlations between depression and bias, and these correlations were only marginally smaller in magnitude than the correlations between anxiety and bias. Thus, the results of three of the five comparisons were in line with prediction, and two were ambiguous.

The second way of examining the hypothesis comparing anxiety to depression is to look at the partial correlation between each mood measure and bias, controlling for the other mood measure. This is a more rigorous test, in that a significant correlation demonstrates an effect that is independent of the other aspect of mood, but given that anxiety and depression are highly correlated, it is also an approach with limited statistical power. In only one case out of the five tasks was a partial correlation statistically significant. However, this partial correlation was in line with prediction - bias on the word stem task was significantly associated with anxiety, even controlling for depression. Moreover, the significance of this finding is unlikely to be a chance result of conducting several analyses, because it is highly significant $(\mathrm{p}<.001)$. Finally, in light of the limited statistical power of this kind of comparison, it is noteworthy than in every case other than this significant finding, there is a nonsignificant trend in the predicted direction. The partial correlations between the mood measure that the model predicts should be more important and bias, controlling for the mood measure predicted to be less important, has in each case a p of .16 or less ${ }^{5}$. The converse partial correlations have in each case a p of .30 or more. Thus, the overall pattern of results is consistent with Williams et al.'s (1997) hypothesis

\footnotetext{
${ }^{5}$ The combined probability of the four non-significant tests obtaining even this very marginal level of significance by chance alone is $0.16^{4}$, or $\mathrm{p}<.001$.
} 
Emotion-Congruent Information Processing 120

that tasks that rely on elaboration are affected by depression, and tasks that rely on priming are affected by anxiety. 
Table 4.

Summary Comparison of the Correlations of Anxiety and Depression with Cognitive Bias Scores.

\begin{tabular}{|c|c|c|c|c|c|}
\hline Task & $\begin{array}{l}\text { Explicit } \\
\text { memory } \\
\text { (Experiment } \\
\text { 1) }\end{array}$ & $\begin{array}{l}\text { Autobiographical } \\
\text { memory } \\
\text { (Experiment 2) }\end{array}$ & $\begin{array}{l}\text { Attention } \\
\text { Deployment } \\
\text { (Experiment } \\
\text { 1) }\end{array}$ & $\begin{array}{l}\text { Perceptual } \\
\text { implicit } \\
\text { memory } \\
\text { (word } \\
\text { stem) }\end{array}$ & $\begin{array}{l}\text { Homophone } \\
\text { interpretation } \\
\text { (Experiment } \\
\text { 2) }\end{array}$ \\
\hline $\begin{array}{l}\text { Measure of } \\
\text { emotion }\end{array}$ & $\begin{array}{l}\text { Negative } \\
\text { words } \\
\text { recalled } \\
\text { (with } \\
\text { positive } \\
\text { words } \\
\text { recalled as a } \\
\text { covariate) } \\
\end{array}$ & $\begin{array}{l}\text { Mean self-rated } \\
\text { pleasantness of } \\
\text { events }\end{array}$ & $\begin{array}{l}\text { Percentage } \\
\text { of trials } \\
\text { coloured bar } \\
\text { replacing } \\
\text { sad word } \\
\text { selected }\end{array}$ & $\begin{array}{l}\text { Primed } \\
\text { positive } \\
\text { target } \\
\text { words } \\
\text { produced } \\
\text { (adjusted } \\
\text { for } \\
\text { baseline) } \\
\end{array}$ & $\begin{array}{l}\text { Number of } \\
\text { negative } \\
\text { homophone } \\
\text { interpretations }\end{array}$ \\
\hline $\begin{array}{l}\text { Depression } \\
\text { (BDI - II) }\end{array}$ & $\begin{array}{l}\mathrm{pr}=.120^{* *} \\
\mathrm{p}=.047 \\
\underline{\mathrm{df}}=194\end{array}$ & $\begin{array}{l}\underline{\mathrm{r}}=-.145^{* *} \\
\underline{\mathrm{p}}=.050 \\
\underline{\mathrm{n}}=130\end{array}$ & $\begin{array}{l}\underline{\mathrm{r}}=.172^{* *} \\
\mathrm{p}=.050 \\
\underline{\mathrm{n}}=93\end{array}$ & $\begin{array}{l}\underline{\mathrm{r}}=-.009 \\
\mathrm{p}=.453 \\
\underline{\mathrm{n}}=193\end{array}$ & $\begin{array}{l}\underline{\mathrm{r}}=.167^{* *} \\
\mathrm{p}=.041 \\
\underline{\mathrm{n}}=110\end{array}$ \\
\hline $\begin{array}{l}\text { Anxiety } \\
\text { (HAD - A } \\
\text { Expt 1, } \\
\text { BAI Expt } \\
\text { 2) }\end{array}$ & $\begin{array}{l}\mathrm{pr}=.090 \\
\underline{\mathrm{p}}=.106 \\
\underline{\mathrm{df}}=191\end{array}$ & $\begin{array}{l}\underline{\mathrm{r}}=-.098 \\
\underline{\mathrm{p}}=.134 \\
\underline{\mathrm{n}}=129\end{array}$ & $\begin{array}{l}\underline{\mathrm{r}}=.195^{* *} \\
\underline{\mathrm{p}}=.031 \\
\underline{\mathrm{n}}=92\end{array}$ & $\begin{array}{l}\underline{\underline{r}}=.189^{* * *} \\
\underline{p}=.004 \\
\underline{n}=193\end{array}$ & $\begin{array}{l}\underline{\underline{r}}=.188^{* *} \\
\underline{\underline{p}}=.025 \\
\underline{\mathrm{n}}=109\end{array}$ \\
\hline $\begin{array}{l}\text { Partial } \\
\text { correlation } \\
\text { with } \\
\text { depression } \\
\text { controlling } \\
\text { for anxiety }\end{array}$ & $\begin{array}{l}\mathrm{pr}=.083 \\
\underline{\mathrm{p}}=.123 \\
\underline{\mathrm{df}}=190\end{array}$ & $\begin{array}{l}\mathrm{pr}=-.107 \\
\mathrm{p}=.114 \\
\underline{\mathrm{df}}=126\end{array}$ & $\begin{array}{l}\mathrm{pr}=.055 \\
\mathrm{p}=.303 \\
\underline{\mathrm{df}}=89\end{array}$ & $\begin{array}{l}\underline{\mathrm{pr}}=-.199^{\psi} \\
\underline{\mathrm{df}}=187\end{array}$ & $\begin{array}{l}\mathrm{pr}=.045 \\
\mathrm{p}=.320 \\
\underline{\mathrm{df}}=106\end{array}$ \\
\hline $\begin{array}{l}\text { Partial } \\
\text { correlation } \\
\text { with } \\
\text { anxiety } \\
\text { controlling } \\
\text { for } \\
\text { depression }\end{array}$ & $\begin{array}{l}\mathrm{pr}=.022 \\
\mathrm{p}=.381 \\
\underline{\mathrm{df}}=190\end{array}$ & $\begin{array}{l}\mathrm{pr}=.009 \\
\underline{\mathrm{df}}=126\end{array}$ & $\begin{array}{l}\mathrm{pr}=.122 \\
\mathrm{p}=.125 \\
\underline{\mathrm{df}}=89\end{array}$ & $\begin{array}{l}\mathrm{pr}= \\
.270^{* * * *} \\
\mathrm{p}=.000 \\
\underline{\mathrm{df}}=187\end{array}$ & $\begin{array}{l}\mathrm{pr}=.097 \\
\mathrm{p}=.160 \\
\underline{\mathrm{df}}=106\end{array}$ \\
\hline
\end{tabular}

${ }^{* *}$ indicates significant at $\mathrm{p}<.05 .^{* * *}$ indicates significant at $\mathrm{p}<.01$. $^{* * * *}$ indicates significant at $\mathrm{p}<.001$. All t-tests were one-tailed. $\mathrm{p}$ values for correlations in the direction opposite to that predicted are not reported as one-tailed t-tests have no statistical power in this circumstance. The use of two-tailed t-tests would have produced one additional significant finding, marked ${ }^{\psi}$, as noted in the results section of Experiment 1. This table shows the results for those tasks where a significant association between bias and either anxiety or depression was found. Results to the left of the grey line are for tasks where it was predicted bias would be more closely associated with depression than with anxiety; results to the right of the grey line are for tasks where it was predicted that bias would be more closely associated with anxiety than with depression. 
Relevant to Aim 2. The second main aim of this thesis was to test the hypothesis that it is the somatic, rather than the cognitive, symptoms of depression that are related to information processing biases. Table 5 summarises the overall pattern of results for tasks that showed a relationship to depression in Experiments 1 and 2. There are two ways of examining the hypothesis. The first is to look at whether the somatic and cognitive factors of depression correlate significantly with bias score. Table 5 shows that in three of the four cases, the somatic factor of depression is significantly correlated with bias; in the other case, the association is near significant. Conversely, in only one case is the cognitive factor of depression significantly correlated with bias, and in only one other case does the association near significance.

The second way of examining the hypothesis comparing the two factors of depression is to look at the partial correlation between each factor score and bias, controlling for the other factor. This is a more rigorous test, in that a significant correlation demonstrates an effect that is independent of the other factor, but given that the two factors are highly correlated, it is also an approach with limited statistical power. Table 5 shows that in the case of the deployment of attention task, the correlation between the somatic factor and bias remains significant controlling for the cognitive factor. Also, in the case of the autobiographical pleasantness task, the correlation between the somatic factor and bias is near significant, controlling for the cognitive factor. Conversely, in no case does the correlation between the cognitive factor and bias near significance, controlling for the somatic factor. Thus, the overall pattern of results is consistent with the hypothesis that depression-congruent cognitive biases are correlates of the somatic, rather than the cognitive, symptoms of depression. 
Table 5.

Summary Comparison of the Correlations of Somatic and Cognitive Factors of Depression with Cognitive Bias Scores.

\begin{tabular}{|c|c|c|c|c|}
\hline$\underline{\text { Task }}$ & $\begin{array}{l}\text { Explicit memory } \\
\text { (Experiment 1) }\end{array}$ & $\begin{array}{l}\text { Attention Deployment } \\
\text { (Experiment 1) }\end{array}$ & $\begin{array}{l}\text { Autobiographical } \\
\text { memory (Experiment 2) }\end{array}$ & $\begin{array}{l}\text { Homophone } \\
\text { interpretation } \\
\text { (Experiment 2) }\end{array}$ \\
\hline Measure of depression & $\begin{array}{l}\text { Negative words recalled } \\
\text { (with positive words } \\
\text { recalled as a covariate) }\end{array}$ & $\begin{array}{l}\text { Percentage of trials } \\
\text { coloured bar replacing } \\
\text { sad word selected }\end{array}$ & $\begin{array}{l}\text { Mean self-rated } \\
\text { pleasantness of events }\end{array}$ & $\begin{array}{l}\text { Number of negative } \\
\text { homophone } \\
\text { interpretations }\end{array}$ \\
\hline BDI - II & $\begin{array}{l}\mathrm{pr}=.120^{* *} \\
\mathrm{p}=.047 \\
\mathrm{df}=194\end{array}$ & $\begin{array}{l}\underline{\mathrm{r}}=.172^{* *} \\
\underline{\mathrm{p}}=.050 \\
\underline{\mathrm{n}}=93\end{array}$ & $\begin{array}{l}\underline{\mathrm{r}}=-.145^{* *} \\
\mathrm{p}=.050 \\
\underline{\mathrm{n}}=130\end{array}$ & $\begin{array}{l}\underline{\mathrm{r}}=.167^{* *} \\
\mathrm{p}=.041 \\
\underline{\mathrm{n}}=110\end{array}$ \\
\hline BDI - II somatic factor & $\begin{array}{l}\overline{\mathrm{pr}}=.124^{* *} \\
\mathrm{p}=.042 \\
\underline{\mathrm{df}}=194\end{array}$ & $\begin{array}{l}\underline{\mathrm{r}}=.229^{* *} \\
\mathrm{p}=.014 \\
\underline{\mathrm{n}}=93\end{array}$ & $\begin{array}{l}\underline{\mathrm{r}}=-.164^{* *} \\
\underline{\mathrm{p}}=.031 \\
\underline{\mathrm{n}}=130\end{array}$ & $\begin{array}{l}\underline{\mathrm{r}}=.144^{*} \\
\mathrm{p}=.067 \\
\underline{\mathrm{n}}=110\end{array}$ \\
\hline BDI - II cognitive factor & $\begin{array}{l}\overline{\mathrm{pr}}=.095^{*} \\
\underline{\mathrm{p}}=.093 \\
\underline{\mathrm{df}}=194\end{array}$ & $\begin{array}{l}\underline{\mathrm{r}}=.082 \\
\underline{\mathrm{p}}=.217 \\
\underline{\mathrm{n}}=93\end{array}$ & $\begin{array}{l}\underline{\mathrm{r}}=-.108 \\
\mathrm{p}=.110 \\
\underline{\mathrm{n}}=130\end{array}$ & $\begin{array}{l}\underline{\underline{r}}=.176^{* *} \\
\underline{\mathrm{p}}=.033 \\
\underline{\mathrm{n}}=110\end{array}$ \\
\hline $\begin{array}{l}\text { Partial correlation with somatic } \\
\text { factor controlling for cognitive } \\
\text { factor }\end{array}$ & $\begin{array}{l}\mathrm{pr}=.082 \\
\underline{\mathrm{p}}=.128 \\
\underline{\mathrm{df}}=193\end{array}$ & $\begin{array}{l}\underline{\mathrm{pr}}=.240^{* *} \\
\underline{\mathrm{p}}=.011 \\
\underline{\mathrm{df}}=90\end{array}$ & $\begin{array}{l}\underline{\mathrm{pr}}=-.131^{*} \\
\underline{\mathrm{p}}=.069 \\
\underline{\mathrm{df}}=127\end{array}$ & $\begin{array}{l}\underline{\mathrm{pr}}=-.001 \\
\underline{\mathrm{df}}=107\end{array}$ \\
\hline $\begin{array}{l}\text { Partial correlation with cognitive } \\
\text { factor controlling for somatic factor }\end{array}$ & $\begin{array}{l}\underline{\mathrm{pr}}=.016 \\
\underline{\mathrm{p}}=.410 \\
\underline{\mathrm{df}}=193\end{array}$ & $\begin{array}{l}\underline{\mathrm{pr}}=-.111 \\
\underline{\mathrm{df}}=90\end{array}$ & $\begin{array}{l}\underline{\mathrm{pr}}=.043 \\
\underline{\mathrm{df}}=127\end{array}$ & $\begin{array}{l}\underline{\mathrm{pr}}=.102 \\
\mathrm{p}=.147 \\
\underline{\mathrm{df}}=107\end{array}$ \\
\hline
\end{tabular}

indicates near significance $(\mathrm{p}<.10){ }^{* *}$ indicates significant at $\mathrm{p}<.05$. All $\mathrm{t}$-tests were one-tailed. $\mathrm{p}$ values for correlations in the direction opposite to that predicted are not reported as one-tailed t-tests have no statistical power in this circumstance. The use of two-tailed t-tests would not have produced any additional significant findings. Correlations and partial correlations between the somatic and cognitive factors of depression and bias were only calculated for cases where there was a significant correlation between total depression score and bias. 


\section{Discussion}

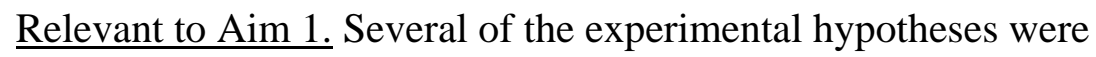
supported. Autobiographical memory valence was significantly associated with depression, but not with anxiety. Partial correlations produced a nonsignificant pattern consistent with this conclusion. Also as predicted, the negative interpretation of negative-neutral homophones was significantly related to anxiety. Negative interpretation was also significantly related to depression. Partial correlations were unable to disentangle the effects of depression and anxiety, but there was a trend supporting the prediction that anxiety would mediate the association. Inconsistent with prediction, there was no significant association between either anxiety or depression and interpretation of neutralpositive homophones. This nonreplication was particularly surprising given that insufficient power is a relatively unlikely explanation of the finding, and that the neutral-positive homophone interpretation was related to scores on the PT, suggesting that individual differences in emotional interpretation were being tapped by the task.

The results of three of the tasks were not consistent with the hypotheses. Bias in conceptual implicit memory was expected to relate to depression, but not to anxiety; contrary to prediction, it related to neither. When this finding is considered in conjunction with the results of the conceptual implicit memory task in Experiment 1, it suggests that clinical levels of depression may be required to produce implicit memory bias. It was also predicted that bias in the two explicit memory tasks would relate to depression, but not to anxiety; again, it related to neither. This was unexpected; the two explicit memory tasks in Experiment 2 
were intended to improve on the methodology of the explicit memory task in Experiment 1, which did find a depression-congruent bias. As discussed in the results, lack of statistical power is a relatively unlikely explanation for the null findings, and as discussed in the introduction, a depression-congruent bias in explicit memory is a relatively well-established phenomenon. On the other hand, also as discussed in the introduction, there have been a number of other studies, with both clinical and nonclinical participants, that have not found depressioncongruent explicit memory biases. It is difficult to determine how many of these failures to replicate were due to insufficient statistical power and how many were due to methodological differences. The meta-analysis conducted by Matt et al. (1992) found no significant evidence that methodological differences influenced effect sizes, but it could be that a meta-analysis incorporating the larger database of studies that now exists would find different results. In the case of the present study, one could speculate that the incidental recall task was contaminated by the implicit memory task that intervened between study and test. Similarly, because the intentional memory task had a greater number of word stimuli than has been commonly used for intentional (rather than incidental) recall tasks, one could speculate that this factor may have affected the results. Mapping out the exact methodological conditions under which depression-congruent recall occurs is a difficult task, because the small effect size involved requires large numbers for sufficient power; it will doubtless be a subject of further research.

Relevant to Aim 2. Some support was obtained for the hypothesis that the somatic, rather than cognitive, symptoms of depression are related to information processing bias. As predicted, the somatic factor of depression was significantly associated with the production of negative autobiographical memories, but the 
cognitive factor was not. Partial correlations produced a near-significant pattern consistent with this conclusion. However, contrary to prediction, the cognitiveaffective factor of depression was significantly associated with negative homophone interpretation, but the somatic factor was only near-significantly associated with this variable. Partial correlations were nonsignificant, but the trend was for the cognitive-affective factor to have the greater influence on interpretation. There are two possible interpretations of this finding. First, the somatic mediation of the effects of depression on cognitive bias may apply to attention and memory, but not to interpretation. Second, the result may have occurred because anxiety mediates the relationship between scores on depression inventories and interpretative bias; however, as described above, there was no significant evidence for this later hypothesis in the current experiment.

Relevant to Subsidiary Aim 2. The relationships of the mood and other variables with autobiographical memory specificity were as predicted. Neither anxiety nor depression was related to autobiographical memory specificity. Both right hemispheric thinking style and poor explicit word list recall were significantly and independently associated with overgeneral memory. Unexpectedly, there was also a significant association between number of overgeneral memories produced and mean pleasantness of recalled events.

Relevant to Subsidiary Aim 3. The relationship between thinking style and homophone interpretation was consistent with prediction. Right-hemispheric thinking style was significantly associated with negative interpretations, near significantly associated with positive interpretations, and significantly associated with combined number of emotional interpretations. Thus, right-hemispheric 
thinking style appears to be associated with the interpretation of ambiguous material as emotional, regardless of valence.

\section{General Discussion}

\section{Implications of Findings Relevant to Aim 1}

To address the issue of the differential information processing correlates of anxiety and depression, it is useful to summarise the overall pattern of relevant results. These results were summarised in Table 4, and they showed support for the hypothesis that anxiety is associated with cognitive biases in tasks requiring priming, and depression is associated with cognitive biases in tasks requiring elaboration. The pattern of findings was consistent with the model put forward by Williams et al. (1997). The schema models of Beck et al. (1979) and Horowitz et al. (1997), and the network model of Bower (1981), do not predict or explain the results.

Some caveats apply to this interpretation of the data. Although the overall pattern of results is very consistent with Williams et al.'s (1997) model, the partial correlations between anxiety and depression were significant in only one case. This means that although the overall conclusion can be confidently drawn, the application of the general rule to some of the specific cases is uncertain. For example, although as predicted performance on the deployment of attention task was more strongly related to anxiety than to depression, this difference was not significant, and other research has produced inconsistent findings on this issue. Unfortunately, the use of partial correlations between highly correlated variables 
is a technique that requires large sample sizes to obtain sufficient statistical power. The present studies had larger samples than most experiments in the area, but the results suggest that obtaining sufficient power to definitively test the hypothesis would require an even larger sample. In some cases, careful attention to methodological detail might also increase statistical power. For example, in the homophone interpretation task, there were several items on which one of the two response options dominated, with more than $90 \%$ of participants responding in that way. It is unlikely that these items were involved in the effects that were found; therefore, replacing them might increase the effect size and thus statistical power. Homophones for which the two possible interpretations have similar word frequencies may be particularly useful.

In a similar vein, several of the tasks used did not relate to either anxiety or depression. As noted in the results, insufficient power is a relatively unlikely explanation of these failures to replicate. Future research should examine the exact parameters within which an effect will or will not be found; this may shed light on when cognitive biases are and are not likely to be exhibited in real-world situations.

A contentious issue, discussed in the introduction, is the generalisation of nonclinical research to clinical populations. As also discussed, a nonclinical population is ideally suited to the current research questions, because in clinical populations anxiety and depression, and somatic and cognitive symptoms of depression, will tend to be confounded. Regardless of whether they are relevant to clinical populations, the present results are relevant to the understanding of cognition and emotion in general. It would be inappropriate to talk about the present findings as if the experiments were measuring generalised "distress"; 
subclinical anxiety and depression showed different correlates, and these differences were broadly consistent with those that would be expected in clinical populations. However, as well as being relevant to understanding basic emotional functioning, there are reasons to believe that the findings of this project are relevant to theory and practice in clinical psychology. First, as discussed in the introduction, the bulk of evidence supports the idea that depression and anxiety are continuous entities. Second, these experiments used the strict depression score cut-offs proposed by Kendall et al. (1987) to classify participants as dysphoric or dysphoric-depressed. In addition, Experiment 2 used the multiplegating procedure that is recommended as best practice for identifying participants who have enduring problems with depression (Ingram \& Hamilton, 1999) participants were first prescreened for high or low levels of depression, and then only classified as dysphoric-depressed if they still showed high levels of distress at the time of the experimental session. There was evidence that the use of these procedures identified a group of participants for whom depression was a problem: 25\% of dysphoric participants and 29\% of dysphoric-depressed participants had undergone formal treatment for depression within the past year. Given that epidemiological studies have shown that less than $50 \%$ of people who meet the criteria for clinical depression in a structured interview have received formal treatment for depression (Angst, 1992; Lecrubier, 1998), these results suggest that the participants in the dysphoric-depressed group represent a significantly distressed population. Thus it seems reasonable to suggest that the present results are relevant to theory and practice in clinical psychology. Nevertheless, it would be useful to replicate these experiments in a clinical population, bearing in mind that it may be necessary to use large samples to 
obtain sufficient statistical power to distinguish between the variables of interest. Similarly, naturalistic studies could be used to complement the results of controlled laboratory studies like those of this thesis (Rusting, 1999).

Another contentious issue is that of the causal direction of the relationship between mood and information processing bias. Mood manipulation studies have demonstrated that mood inductions can cause negative biases across a range of tasks (for example, Rusting, 1999). This is evidence that negative emotion can cause processing biases. However, it is also possible that some of the association between information processing and negative mood is caused by a reciprocal effect: information processing influencing mood. As mentioned in the introduction, researchers have shown a strong interest in this possibility. However, although researchers into cognitive biases usually speculate as to whether cognitive biases may have some causal effect, direct empirical investigation of the possibility has been surprisingly infrequent (Mathews \& MacLeod, 2002). MacLeod and Hagan (1992) conducted the first published study on the matter; women who showed more emotional word interference on a masked Stroop task showed greater anxiety and depression in response to a negative life event. Van den Hout, Tenney, Huygens, Merckelbach, and Kindt (1995) conducted a conceptual replication of this study, although they used an ad hoc self-rating questionnaire to measure vulnerability, and provided no evidence that this questionnaire actually predicts future emotional distress. Rude, Wenzlaff, Gibbs, Vane, and Whitney (2002) conducted a study that examined cognitive biases on a task involving unscrambling sentences. Cognitive biases at the time of the experiment (Time 1) were compared to depression scores obtained four to six weeks later (Time 2). Cognitive biases on the task were 
related to symptoms of depression at Time 2, controlling for depression at Time 1. However, because each of these studies used a correlational design, it remains possible that a third variable causes both information processing bias and vulnerability to later depression and anxiety (Mathews \& MacLeod, 2002). Rude et al. (2002) did not control for anxiety at Time 1, and given that anxiety is associated with both biased judgement and later depression (Mineka, Watson, \& Clark, 1998), this makes interpretation of the study ambiguous. A similar criticism applies to the MacLeod and Hagan (1992) and Van den Hout et al. (1995) studies: personality predicts both current information processing biases and future vulnerability to depression and anxiety (Rusting, 1999). Mathews and MacLeod (2002) review a series of studies that address these concerns, providing the strongest evidence so far that information processing biases can causally influence mood. In these experiments, processing biases are induced by reinforcing bias with better performance on a task. Participants for whom negative bias is induced show a heightened negative mood reaction to a stress task, compared with participants for whom positive bias is induced. This appears to demonstrate induced cognitive vulnerability to negative emotion. This series of studies should be interpreted with caution at this stage, because the bulk of the studies have yet to be published and subjected to public scrutiny. In sum, the existing body of research seems to suggest a reciprocal relationship between processing bias and negative emotion, but research demonstrating that negative biases cause negative emotion is best considered preliminary at this stage.

Because the present experiments were correlational in nature, causal direction cannot be inferred from their results. This thesis has suggested that anxiety and depression have different effects on different information processes. 
However, it is also possible that biases in different information processing systems have different effects on anxiety and depression: for example, negative biases in attention may lead to anxiety, while negative biases in memory may lead to depression. Finally, both explanations may apply. Studies that examine the effects of inducing biases in different kinds of processes on anxiety versus depression would help to resolve this issue. Likewise, studies that specifically induce anxious or depressed mood, rather than generally negative affect, could examine any subsequent differences in the kinds of information processing bias that are shown.

Experiment 2 had particular methodological strengths that improve over much existing research in the area. Demand effects have been cited as a possible weakness in many existing studies (Blaney, 1986). In Experiment 2, an effort was made to disguise the purpose of the tasks, and the question about understanding of the experimental purpose showed that this effort was largely successful. Participants were excluded if they evidenced understanding of the mood-related nature of the tasks, but supplementary analyses showed that the results would have been similar without this exclusion process. These findings also support the validity of experiments that have not measured participant understanding of the experimental purpose, including Experiment 1.

In addition to demand effects, response bias has also been suggested as a possible alternative explanation of mood-congruency in homophone tasks (MacLeod, 1990) and explicit memory tasks (Blaney, 1986). However, response bias and demand explanations predict that depression and anxiety should both result in a generally negative response style across all kinds of tasks. This prediction is inconsistent with the finding that only depression produced negative 
bias in explicit and autobiographical memory, but that both depression and anxiety produced a negative interpretative bias on the emotional homophone task. There were also cases where depressed and anxious participants had a clear opportunity to exhibit a negative response style, but did not (for example, the two explicit memory tasks in Experiment 2). Thus, response bias and demand effects provide poor explanations of the overall pattern of results across the two experiments.

Tests of bias in autobiographical memory are often criticised on the grounds that any group differences shown could be due to veridical differences in life history, rather than to information processing bias. The autobiographical memory task used in Experiment 2 did not control for life history differences, but the veridical difference explanation does not explain why negative recall was related to the somatic factor of depression, rather than to the cognitive factor or to anxiety. On the other hand, the pattern of information processing biases hypothesised in Aims 1 and 2 does predict and explain the results, suggesting that information processing biases played a part in producing the results. Although controlling for veridical differences was beyond the scope of a multiple-task experiment like Experiment 2, future research could do so, for example by examining recall of high school grades and checking this against school records (Bahrick, Hall, \& Berger, 1996).

The interaction between anxiety and depression in the perceptual implicit memory task was particularly interesting. On this task, there was no significant correlation between depression and negative implicit memory, but there was a positive correlation between anxiety and implicit memory for negative material, and this correlation increased in magnitude when depression was controlled for 
using a partial correlation. The correlation between depression and implicit memory for negative material became negative when anxiety was controlled for. Although this correlation was not significant, because it was tested using a onetailed t-test intended to detect relationships in the other direction, it was sizable in magnitude. Taken at face value, this finding would suggest that "pure" depression is associated with a positive bias in perceptual implicit memory. These results appear to parallel those found by Lawson and MacLeod (1999) using a test of interpretation of ambiguity. Those authors controlled for the effects of anxiety by selecting participants who were equal on a measure of anxiety but differed on a measure of depression, and they found that those participants who were more depressed showed a positive bias. The current experiment obtained equivalent results controlling for depression statistically, rather than methodologically. As detailed in the introduction, one way to interpret these results is to postulate that these biases are related to pure anxiety, and not at all to general negative affectivity or depression. This interpretation of the results explains the remarkable consistency with which past studies have found that perceptual implicit memory biases are related to anxiety, but not to depression. In contrast, attentional biases have shown an inconsistent relationship with depression; this may be because these biases are influenced by both anxiety and general negative affectivity.

\section{Implications of Findings Relevant to Aim 2}

To address the issue of the relative effects of the somatic and cognitive aspects of depression, it is useful to summarise the overall pattern of relevant results. These results were summarised in Table 5, and they showed support for 
the hypothesis that the somatic, rather than cognitive, symptoms of depression are related to information processing bias. Although this conclusion is supported by the overall pattern of findings, the significance of the comparisons varied from case to case, and by method of analysis. In particular, the results from the homophone interpretation task were not consistent with the hypothesis. As already discussed, this may be because bias on the homophone task is not directly related to depression. However, it may simply be that interpretative bias is an exception to the general pattern that was observed; somatic symptoms may not influence interpretation more than cognitive symptoms. A similar limitation is that the results were found with only a limited range of tasks; investigating a wider range of tasks will allow investigators to determine how general the effect is. Finally, the current study did not compare the effects of the somatic and cognitive symptoms of anxiety on information processing, because the anxiety inventories used did not have factor structures that divide the symptoms this way (Bjelland et al., 2002; Osman, Barrios, Aukes, Osman, \& Markway, 1993). Future research could investigate this issue using an inventory designed to differentiate the cognitive and somatic symptoms of anxiety, for example, the Cognitive-Somatic Anxiety Questionnaire (Schwartz, Davidson, \& Goleman, 1978).

A possible alternative interpretation of the findings is that the somatic subscale of the BDI-II is a more valid measure of general depression than the cognitive scale. This interpretation seems unlikely. The somatic scale contains eleven items, and the cognitive-affective scale ten; given that the BDI-II has excellent psychometric properties this means that it is unlikely that one subscale is substantially more reliable than the other. Similarly, the somatic symptoms of 
depression (for example, weight change or sleep disturbance) do not have greater face validity as measures of depression than do the cognitive symptoms (for example, perceiving the self as a failure). Thus, the most plausible interpretation of the results is that the greater influence of somatic symptoms is genuine. The findings could be further strengthened by using a variety of depression scales to compare the relationship between somatic and cognitive symptoms and information processing bias. The results also suggest that existing self-report scales that contain a predominance of somatic items, for example, the Inventory to Diagnose Depression (Zimmerman \& Coryell, 1987), might be particularly suited to investigations of information processing bias in depression.

The strong association between somatic symptoms and cognitive biases may help explain why studies have found discontinuities between nonclinical and clinical samples. The DSM-IV criteria used to diagnose depression in studies of clinical depression are predominantly somatic (Gotlib \& Hammen, 1992). In contrast, many self-report inventories, for example the frequently-used BDI and BDI-II, also contain many cognitive symptoms (Basco et al., 1997). In combination with the greater symptom severity shown by clinical populations, the predominantly somatic content of the DSM-IV criteria may mean that studies using these criteria are more likely to find evidence of cognitive biases.

As discussed earlier, ideally the present results should be replicated in a clinical population, but large sample sizes might be necessary to obtain enough statistical power to make the results of such an experiment meaningful. Also as noted in the discussion of Aim 1, it is worth considering the causal direction of the relationship between information processing bias and mood. Because these studies were correlational, they do not show whether the somatic symptoms of 
depression have a greater influence on information processing biases than do the cognitive symptoms, or if the relationship is the opposite: information processing biases have a greater influence on the somatic symptoms of depression than on the cognitive. However, there is theoretical and empirical support for the first interpretation, but no obvious theoretical explanation accommodates the second interpretation. In particular, the study by Kumari et al. (1998) showed that a biological intervention caused negative biases in autobiographical memory, and that this effect was not wholly mediated by changes in cognitive symptoms (selfrated mood). These conclusions could be confirmed by manipulating information processing biases and examining whether somatic or cognitive symptoms of depression are more strongly affected. If manipulating bias does not affect somatic symptoms more, support would be obtained for the hypothesis that the causal direction is from somatic symptoms to information processing biases.

The findings relevant to Aim 2 suggest that prevailing models of information processing bias in anxiety and depression are incomplete or incorrect. Schema and network theories predict that cognitive symptoms of depression will influence information processing more than somatic symptoms. The pattern of results shown in the current project was diametrically opposed to this prediction. If this pattern is consistently replicable, then future models of information processing bias will need to explicitly include physiological processes. The final section of this thesis discusses how such a model might work. 


\section{Implications of Findings Relevant to Subsidiary Aim 1}

Experiment 1 examined whether positive biases on the DOAT are characteristic of the general population, as previous authors have concluded, or if they are shown by only a minority of people. Previous research selected control participants based on particularly low depression or anxiety scores; the present study took an unselected sample and examined the responses of participants from each quartile of anxiety and depression. Only participants who were in the lower $50 \%$ of depression scores, or the lowest $25 \%$ of anxiety scores, showed a positive bias on the task. These results suggest that it is not that being unusually high in anxiety or depression causes the erosion of a normative positive bias; rather, only people who show a below average amount of negative emotion show a positive bias. The results reinforce the point that control groups in depression research should cover a broad range of normative values rather than being selected for being extremely low in depressive symptomatology (Ingram \& Hamilton, 1999) ${ }^{6}$. The results also suggest that positive attentional biases may be a product of particularly positive mood states; future research could further explore this finding by looking at the relationship between attention and positive mood.

\section{Implications of Findings Relevant to Subsidiary Aim 2}

Experiment 2 examined whether autobiographical specificity was related to a range of factors: anxiety, depression, thinking style, and wordlist memory.

\footnotetext{
${ }^{6}$ The experiments in this thesis accommodated this suggestion by including as the control group all participants who scored beneath thirteen on the BDI-II.
} 
Consistent with prediction, specificity did not relate to self-reported anxiety or depression. Rather it related to thinking style and to wordlist memory ability.

This study has confirmed that nonclinically depressed participants do not show overgeneral memory, but the puzzle discussed in the introduction remains. Why do clinically depressed participants show overgeneral memory, when subclinically depressed participants do not? As discussed in the results, there was no significant difference in specificity of recall between the participants who had recently undergone treatment for depression and the other participants. This suggests that overgeneral memory is not always triggered by serious cases of depression. It also suggests that overgeneral memory is not due to side effects of medication, because the majority of the treated participants were taking medication at or shortly before the time of the study. One possibility is that overgeneral memory in clinical depression is the product of an interaction between depression and IQ, with overgeneral memory only being found in depressed individuals with below average intelligence. This would explain why the effect is not found in student populations, who are generally of above average intelligence (Crawford \& Allan, 1997), but the issue remains a matter for further research.

Specificity of memories was, however, related to wordlist recall ability. This is consistent with the speculation that the processes that cause overgeneral recall may be the same as those that cause poor wordlist memory in depression. However, the relationship was not as strong as might have been expected - a correlation of .20 , compared with a correlation of .25 between thinking style and specificity. It is possible that specificity is related to general intelligence rather than specifically to memory; future research could examine this. An experiment 
designed to examine this particular issue would also benefit from including a task measuring motivation and effort.

Interestingly, the relationship between thinking style and specificity of recall was opposite in direction to that found by Merckelbach et al. (1996). These authors predicted that people with a right-hemispheric dominant thinking style would pay less attention to detail and would thus be more likely to produce overgeneral memories. However, they found the opposite: right-hemispheric thinkers produced more specific memories. The current project found results in line with their original hypothesis: left-hemispheric thinkers produced more specific memories. The main difference between the two studies is that Merckelbach et al. (1996) used only emotional cues to trigger memories, and the current study used only neutral cues. One possible explanation for the pattern of results is that left-hemispheric thinkers could be generally better at reporting specific memories, but right-hemispheric thinkers particularly good at recalling emotion-related information ${ }^{7}$. This hypothesis could be better tested in an experiment that measured thinking style and specificity of recall in response to both neutral and emotional cues. A simple distinction between holistic and analytical thinking (Iaccino, 1993) does not provide an obvious explanation of the contrasting results of the present study and that of Merckelbach et al. (1996), but a more detailed examination of theories of hemispheric specialisation would lead this thesis too far astray from its main aims. This could be an area for future theory development and research.

7 This possibility is supported by evidence that the right hemisphere shows specialization for the processing of emotional information (Mandal et al., 1996). 
Implications of Findings Relevant to Subsidiary Aim 3

As predicted, right-hemispheric thinking was related to the production of more emotional homophones, both positive and negative. This finding is consistent with arguments that the right hemisphere of the brain plays a special role in the processing of emotion (Borod et al., 2002). We measured hemispheric dominance indirectly, using a paper-and-pencil test, but there is evidence that responses on the test do relate to measurable physiological differences (Merckelbach, Muris, Pool et al., 1996). The PT contains very little explicit reference to emotion ${ }^{8}$, which makes the test's relationship with a behavioural index of emotional responding quite striking. Researchers have already started to look at the relationship between brain functioning and positive or negative cognitive biases (Deldin et al., 2001; Gotlib, Ranganath, \& Rosenfeld, 1998); the present data suggest that it would also be productive to look at brain functioning and bias towards emotional material regardless of valence. The interaction between the two forms of biases (positive-negative and nonemotional-emotional) would also be an interesting area of study. Finally, it would be revealing to see whether the PT relates to other emotion-related tasks; for example, righthemispheric dominant individuals may show attentional biases towards emotional material on the DOAT and dot-probe tasks.

\footnotetext{
${ }^{8}$ Of the twenty questions, only three contain emotional content, and this content is tangential. These questions are "Do you base your decisions on objective facts rather than feelings?", "Do you use a playful approach to problem solving?”, and "Do you use a serious, all business approach to problem solving?”
} 


\section{Implications of Findings Relevant to Subsidiary Aim 4}

The overall findings have mixed implications for the possibility of using biases in information processing to estimate level of depression. In each experiment, only two of the five tasks were significantly related to depression, and in each case the relationship was closer to small than to moderate in Cohen's (1988) effect size terms, with correlations ranging from .12 to .17 in magnitude. This means that neither of the two task batteries examined in the current project would, by themselves, be useful measures of depression. If the four depressionrelated tasks were put together in one experiment, and similar correlations between depression and bias were obtained, the resulting battery could be more useful, but probably only for group, rather than individual, comparisons; this possibility requires investigation. Even a battery that was only reliable when looking at differences between large groups could be useful for theoretical research in areas where differences in willingness to report symptoms are suspected to be an issue, for example, examinations of gender differences. It might also be possible to increase the tasks' relationships with depression using methodological refinements, as discussed under Aim 1. When compared to some results that have been reported in the literature, like the correlation between bias and depression of .44 reported by Dalgleish (1995), these results are disappointing. However, in retrospect this was to be expected; the published literature is likely to be biased in favour of significant findings, and given small sample sizes this means large effect sizes (Meehl, 1990). Also, some strategies that have been used to select participants are likely to inflate obtained effect sizes, compared to those that would be found using an unselected sample (Coyne, 
1994). In sum, developing an information processing measure of depression remains an elusive goal. Reaching it will require continuing to refine different tasks, and then eventually conducting large-scale studies.

\section{Implications of the Overall Project}

The main aims of the project involved comparing the effects of anxiety and depression, and comparing the effects of the somatic and cognitive symptoms of depression. Both areas of investigation produced results that can not be easily explained by the traditional cognitive models (Beck et al., 1979; Bower, 1981; Horowitz et al., 1997). Can more recent models of cognition and emotion provide a better fit to the data? This section describes how they might.

It is worth emphasizing that cognitive-level models are essential to the explanation of cognitive biases in emotion, and that the present results do not argue against this. Modelling the form and function of information processing and describing the biological structures that carry out this processing are related but distinct and equally important enterprises ${ }^{9}$ (Ilardi \& Feldman, 2001; Teasdale, 1999). Rather, cognitive models will need to include explanations of the differences between anxiety and depression, and of the close association between the somatic symptoms of depression and processing bias. Certain recent cognitive models are able to accommodate such explanations. As discussed in the introduction, Williams et al. (1997) have proposed a model of how depression and anxiety affect information processing. They also described how this model

\footnotetext{
${ }^{9}$ Some have argued that findings from biological neuroscience are not relevant to the understanding of how the mind works (Coltheart, 2002). However, others strongly disagree (Ilardi \& Feldman, 2001). For present purposes, it is sufficient to note that it is difficult to provide an account of the close association of the somatic symptoms of depression with information processing bias without discussing the biology of the brain.
} 
could fit within each of two larger models of information processing, the Multiple-Entry Memory system (MEM) and Interacting Cognitive Subsystems (ICS). Teasdale (1999) has drawn parallels between ICS and what is known about the brain and emotion, and so it is possible to map Williams et al.'s (1997) explanation on to biological structures. As discussed below, this two-level model also provides a possible explanation for the close association between the somatic symptoms of depression and information processing bias.

ICS is a general model of cognition that has been applied in detail to cognition-emotion relations (Teasdale \& Barnard, 1993). The basic tenet of the model is that cognitions come in qualitatively different forms. Relatively unprocessed perceptual information is represented in sensory codes, and somewhat more processed information is represented in speech and visual object codes. Regularities in speech and visual objects are encoded as meanings. For example, multiple sightings of one object on top of another, combined with hearing the speech code "above" or "on top of" could be used to construct a meaning code signifying the concept of “above” (Teasdale \& Barnard, 1993). Most importantly, meanings can be propositional (cold, rational, explicit) or implicational (hot, implicit) ${ }^{10}$. Emotion is conceptualised as activation of certain implicational meanings. Memories for information in the different mental codes are stored separately, and specific processes are needed to transform information from one type of code to another.

Williams et al. (1997) suggest that the distinct processing biases associated with anxiety and depression could be explained within the ICS model. They suggest that implicational codes for anxiety may be tightly bound to

\footnotetext{
${ }^{10}$ A propositional/implicational distinction has also been proposed by earlier authors, for example, Oatley and Johnson-Laird (1987).
} 
sensory codes; thus, when the anxiety code is activated, sensory processes are affected. In contrast, the implicational codes for depression may be tightly bound to propositional codes; thus, when the depression code is activated, processing of meaning is affected. This explanation is an elaboration and extension of Williams et al.'s $(1988,1997)$ original priming-elaboration distinction.

Cognitive models like ICS provide ideas as to how anxiety and depression might produce different effects on information processing, but they leave unanswered the question of why they should do so. Functional approaches to emotion do suggest such an explanation. Oatley and Johnson-Laird (1987) suggest that anxiety evolved to promote vigilance for danger. Biased attention and judgement serve this goal (Mathews, 1990). It also makes sense that anxiety would work at the perceptual level, serving to rapidly identify dangerous stimuli. This may explain why anxiety affects perceptual implicit memory. Similarly, Oatley and Johnson-Laird (1987) suggest that depression evolved to promote withdrawal from unproductive goals and the establishment of new ones. Biased memory may help with this goal, by forcing the individual to focus on the negative consequences of current patterns of behaviour. It also makes sense that depression would work at the conceptual level, serving to identify behaviours that should be avoided (Williams et al., 1997).

The argument that the functional differences between anxiety and depression are the product of evolution suggests that they may be served by specific, evolved, and phylogenetically old structures in the brain. No-one has yet provided a model of how the different processes that are associated with anxiety and depression might map onto biological structures. However, Teasdale (1999) 
has described how ICS could map onto these structures, which provides a useful start for thinking about the issue. First, the biology.

Gray (1982) based his influential theory of the neuropsychology of anxiety on the concept of a behavioural inhibition system (BIS). The concept of the BIS was based on animal data showing that novel stimuli, or stimuli signalling potential punishment or nonreward, triggered a specific set of responses. Anxiolytic drugs selectively prevented this set of responses. The BIS is the system that produces these responses, and Gray (1982) operationalises anxiety as an increase in activity in the BIS. Gray (1982) describes evidence that the septo-hippocampal system is particularly important in the functioning of the BIS, and more recent evidence has generally supported this idea (Buck, 1999).

The amygdala is a region of the brain that is highly interconnected with the septo-hippocampal system (Buck, 1999). LeDoux (1995, 2000) argues that the amygdala plays a particularly important role in fear. He presents evidence that a specific part of the amygdala processes sensory stimuli and triggers fear reactions. The amygdala receives sensory stimuli in highly processed form from cortical structures, and in less processed form from the thalamic sensory relay structures. The latter route allows rapid responses to signs of threat. Fear conditioning can occur to information received from either source. In addition, the model suggests that the hippocampus interacts with the amygdala to modulate the fear response. This lets conditioned fear responses be affected by context; thus the organism can respond to a stimulus as threatening in one situation, but not in another. Finally, Rolls and Treves (1998) observe that the amygdala has neurons that project back to many of the areas that supply it with information. These authors argue that the physiological characteristics of these 
neurons, and the way that they fit into the organisation of the brain, suggest that they influence recall and attention.

Teasdale (1999) describes how his cognitive ICS model may map onto LeDoux's neuropsychological model. Teasdale’s model is one of cognition and emotion in general, and LeDoux's is primarily a model of fear conditioning, so it might seem to be a stretched comparison. However, Teasdale (1999) argues that LeDoux’s model is likely to be relevant to at least some other emotions. In particular, the amygdala appears to be involved in both anxiety and depression (Buck, 1999), and neuroimaging studies have shown that activity in this area is elevated in both these states (Davidson, Pizzagalli, Nitschke, \& Putnam, 2002).

Teasdale's (1999) mapping between ICS and LeDoux’s model suggests that the amygdala functions to transform sensory or propositional codes to implicational codes. Sensory code is received via the thalamic sensory relay structures, propositional code representing current stimuli is received from cortical structures, and propositional code representing past events is received from the hippocampus. It is useful to consider the joint implications of Williams et al.'s (1997) mapping between their own theory and ICS, Teasdale's (1999) mapping between ICS and LeDoux’s model, and Rolls and Treves' (1998) argument that the amygdala influences information processing. Combining these ideas, it is possible to speculate as to how the different effects of anxiety and depression may be manifested in neural terms. The information processing effects of depression may be mediated at the interfaces between cortical structures and the amygdala, and between the hippocampus and the amygdala. The amygdala does not have the anatomical capacity to send information directly back to the thalamus, but it does back project to early and late sensory processing 
areas (LeDoux, 1995), and it is these projections that may be particularly affected by anxiety. These hypotheses could be tested using functional imaging to examine the activity of the connections between these areas in participants with emotional disorders, as advocated by Davidson et al. (2002).

Thus, we can see how cognitive, evolutionary, and biological explanations of the different effects of anxiety and depression might be constructed and integrated. This framework may also help explain the close association between the somatic symptoms of depression and information processing bias. The hippocampus, the amygdala, and the hypothalamus are often grouped together and called the limbic system ${ }^{11}$ (Buck, 1999). As well as its involvement in anxiety and depression, the limbic system controls drive, experience of pleasure, the endocrine system, sleep, appetite, and libido (Shelton et al., 1991). Thus, it is likely that the limbic system is involved in the somatic symptoms of depression (Shelton et al., 1991). The majority of items contained in the somatic scale of the BDI-II concern bodily functions that are controlled by the limbic system - "loss of pleasure, loss of interest, loss of energy, changes in sleep, changes in appetite, tiredness-fatigue, and loss of interest in sex"12 (Dozois et al., 1998, p. 86). The association between these symptoms and information processing bias suggests that, consistent with Teasdale's (1999) speculation, depression-congruent bias may also be controlled by the limbic system. In this light, the involvement of the hippocampus in explicit memory (Teasdale, 1999), and of the amygdala in threat evaluation (LeDoux, 1995), appear especially interesting.

\footnotetext{
${ }^{11}$ LeDoux (1996) argues that neuroscientists should postulate specific circuits rather than using the term limbic system, but the term is useful for descriptive purposes (Buck, 1999).

${ }^{12}$ The other symptoms loading on this scale are "crying, agitation, and loss of concentration" (Dozois et al., 1998, p. 86).
} 
This has been a necessarily brief and speculative explanation of the present results, but if the results are consistently replicable, then it is an explanation that could be further tested and developed. Future research could also directly examine the relationship between information processing biases and specific brain activity (Davidson et al., 2002). As Teasdale (1999) argues, comparing cognitive and biological levels of analysis should serve to refine and constrain explanations at both levels.

It seems fitting to close by turning to the concerns that inspired Beck's theory, and thus first inspired research into cognition and emotion. What are the implications of the present results for the treatment of anxiety and depression?

Segal, Lau, and Rokke (1999) have suggested that cognitive therapists could conceptualise their treatments as working by counteracting cognitive biases. It may also be helpful to explicitly tell depressed and anxious individuals about the way that their mood states can bias the way that they process information about the world. The present results extend this recommendation, and imply that clinicians can tell depressed clients that these biases are neurobiologically caused. Some clients may find this information helpful or reassuring, because it suggests that the biases are normal biological processes rather than personal failings. (It would also be important to emphasize that the therapist will teach the client cognitive strategies that have been demonstrated to help overcome the biases). The results also suggest that interventions for anxiety may profit by focussing on changing attentional processes, while interventions for depression may profit by focussing on changing memory processes. More speculatively, Mathews and MacLeod (2002) reviewed evidence that experimentally induced information processing biases influenced mood. It may 
be possible to develop this paradigm into a component of a treatment package. If so, it may be that inductions of positive bias in attention and judgement are especially efficacious in the treatment of anxiety, and inductions of positive bias in memory are especially efficacious in the treatment of depression.

This thesis has shown that anxiety and depression have different effects on information processing, and has suggested that the effects of depression on information processing are biologically mediated. This final section has shown that these findings make sense in the context of recent cognitive and biological models of emotion, and also make sense from an evolutionary perspective. Further progress in specifying the relations between information processing and emotion may help integrate cognitive and biological levels of understanding (LeDoux, 2000). Ultimately, this project may help to build a model that can integrate knowledge across areas that are now disparate, and advance both our understanding of ourselves and the treatment of disorders that are so very distressing to so many. 
References

American Psychiatric Association. (1994). Diagnostic and statistical manual of mental disorders (4th ed.). Washington, DC: American Psychiatric Association.

Angst, J. (1992). Epidemiology of depression.

Psychopharmacology, 106(Suppl), 71-74.

Bahrick, H. P., Hall, L. K., \& Berger, S. A. (1996). Accuracy and distortion in memory for high school grades. Psychological Science, 7, 265-271.

Banos, R. M., Medina, P. M., \& Pascual, J. (2001). Explicit and implicit memory biases in depression and panic disorder. Behaviour Research \& Therapy, 39, 61-74.

Basco, M. R., Krebaum, S. R., \& Rush, A. J. (1997). Outcome measures of depression. In H. H. Strupp \& L. M. Horowitz (Eds.), Measuring patient changes in mood, anxiety, and personality disorders: Toward a core battery (pp. 191-245). Washington, DC, US: American Psychological Association.

Beck, A. T., \& Beamesderfer, A. (1974). Assessment of depression: The depression inventory. In P. Pichot (Ed.), Psychological measurements in psychopharmacology. Modern problems in pharmacopsychiatry (Vol. 7, pp. 151-169). Basel, Switzerland: Darger.

Beck, A. T., \& Beck, R. (1972). Screening depressed patients in family practice: A rapid technic. Postgraduate Medicine, 52, 81-85.

Beck, A. T., Rush, A. J., Shaw, B. F., \& Emery, G. (1979). Cognitive therapy of depression. NY: Guilford. 
Beck, A. T., \& Steer, R. A. (1991). Relationship between the Beck Anxiety Inventory and the Hamilton Anxiety Rating Scale with anxious outpatients. Journal of Anxiety Disorders, 5, 213-223.

Beck, A. T., \& Steer, R. A. (1993). Beck Anxiety Inventory

Manual. Orlando: The Psychological Corporation.

Beck, A. T., Steer, R. A., \& Brown, G. K. (1996). BDI - II: Beck

Depression Inventory - Second Edition manual. San Antonio: The Psychological Corporation.

Beidel, D. C., \& Turner, S. M. (1986). A critique of the theoretical bases of cognitive-behavioral theories and therapy. Clinical Psychology Review, 6, 177-197.

Beuke, C. J. (2002). Anxiety, Depression, and Deployment of Attention. Paper presented at the Stress and Anxiety Research Society 23rd International Conference, Melbourne, Australia.

Bieling, P. J., Antony, M. M., \& Swinson, R. P. (1998). The StateTrait Anxiety Inventory, Trait version: Structure and content re-examined. Behaviour Research \& Therapy, 36, 777-788.

Bjelland, I., Dahl, A. A., Haug, T. T., \& Neckelmann, D. (2002). The validity of the Hospital Anxiety and Depression Scale: An updated literature review. Journal of Psychosomatic Research, 52, 69-77.

Blaney, P. H. (1986). Affect and memory: A review. Psychological Bulletin, 99, 229-246.

Borod, J. C., Bloom, R. L., Brickman, A. M., Nakhutina, L., \& Curko, E. A. (2002). Emotional processing deficits in individuals with unilateral brain damage. Applied Neuropsychology, 9, 23-36. 
Bower, G. H. (1981). Mood and memory. American Psychologist, 36, 129-148.

Bradley, B., \& Mathews, A. (1983). Negative self-schemata in clinical depression. British Journal of Clinical Psychology, 22, 173-181.

Bradley, B. P., Mogg, K., \& Millar, N. (1996). Implicit memory bias in clinical and non-clinical depression. Behaviour Research \& Therapy, 34, 865-879.

Bradley, B. P., Mogg, K., Millar, N., \& White, J. (1995). Selective processing of negative information: Effects of clinical anxiety, concurrent depression, and awareness. Journal of Abnormal Psychology, 104, 532-536.

Bradley, B. P., Mogg, K., \& Williams, R. (1994). Implicit and explicit memory for emotional information in non-clinical subjects. Behaviour Research \& Therapy, 32, 65-78.

Bradley, B. P., Mogg, K., \& Williams, R. (1995). Implicit and explicit memory for emotion-congruent information in clinical depression and anxiety. Behaviour Research \& Therapy, 33, 755-770.

Brewin, C. R., Reynolds, M., \& Tata, P. (1999). Autobiographical memory processes and the course of depression. Journal of Abnormal Psychology, 108, 511-517.

Brittlebank, A. D., Scott, J., Williams, J. M., \& Ferrier, I. N. (1993). Autobiographical memory in depression: State or trait marker? British Journal of Psychiatry, 162, 118-121.

Buck, R. (1999). The biological affects: A typology. Psychological Review, 106, 301-336. 
Burt, D. B., Zembar, M. J., \& Niederehe, G. (1995). Depression and memory impairment: A meta-analysis of the association, its pattern, and specificity. Psychological Bulletin, 117, 285 - 305.

Byrne, A., \& Eysenck, M. W. (1993). Individual differences in positive and negative interpretive biases. Personality \& Individual Differences, $\underline{14}, 849-851$.

Carter, C. S., Maddock, R. J., \& Magliozzi, J. (1992). Patterns of abnormal processing of emotional information in panic disorder and major depression. Psychopathology, 25, 65-70.

Clark, D. A., Beck, A. T., \& Alford, B. A. (1999). $\underline{\text { Scientific }}$ foundations of cognitive theory and therapy of depression. New York: John Wiley \& Sons, Inc.

Clark, D. A., \& Teasdale, J. D. (1982). Diurnal variations in clinical depression and accessibility of memories of positive and negative experiences. Journal of Abnormal Psychology, 52, 1090-1097.

Clark, L. A., \& Watson, D. (1991a). Theoretical and empirical issues in differentiating depression from anxiety. In J. Becker \& A. Kleinman (Eds.), Psychosocial aspects of depression (pp. 39-65). Hillsdale, NJ: Lawrence Erlbaum.

Clark, L. A., \& Watson, D. (1991b). Tripartite model of anxiety and depression: Psychometric evidence and taxonomic implications. Journal of Abnormal Psychology, 100, 316-336.

Cohen, J. (1988). Statistical power analysis for the behavioural sciences (2nd ed.). Hillsdale: L Erlbaum Associates. 
Coltheart, M. (2002). Cognitive neuropsychology. In H. Pashler \& J. Wixted (Eds.), Stevens' handbook of experimental psychology (3rd ed.), Vol. 4: Methodology in experimental psychology. (pp. 139-174). NY: John Wiley. Corballis, M. C. (1999). Are we in our right minds? In S. D. Sala (Ed.), Mind myths: Exploring popular assumptions about the mind and brain. (pp. 25-41). Chichester: Wiley.

Coren, S. (1992). The left-hander syndrome: The causes and consequences of left-handedness. New York: The Free Press.

Cox, B. J., Enns, M. W., Borger, S. C., \& Parker, J. D. A. (1999). The nature of the depressive experience in analogue and clinically depressed samples. Behaviour Research \& Therapy, 37, 15-24.

Coyne, J. C. (1994). Self-reported distress: Analog or ersatz depression? Psychological Bulletin, 116, 29-45.

Coyne, J. C., \& Schwenk, T. L. (1997). The relationship of distress to mood disturbance in primary care and psychiatric populations. Journal of Consulting \& Clinical Psychology, 65, 161 - 168.

Crawford, J. R., \& Allan, K. M. (1997). Estimating premorbid WAIS-R IQ with demographic variables: Regression equations derived from a UK sample. Clinical Neuropsychologist, 11, 192-197.

Crits-Christoph, P., \& Connolly, M. B. (1997). Measuring change in patients following psychological and pharmacological interventions: Anxiety disorders. In H. H. Strupp \& L. M. Horowitz (Eds.), Measuring patient changes in mood, anxiety, and personality disorders: Toward a core battery. (pp. 155188). Washington, DC, US: American Psychological Association. 
Dalgleish, T. (1995). Performance on the emotional Stroop task in groups of anxious, expert, and control subjects: A comparison of computer and card presentation formats. Cognition \& Emotion, 9, 341-362.

Dalgleish, T., \& Cox, S. G. (2000). Mood and memory. In G. E. Berrios \& J. R. Hodges (Eds.), Memory disorders in psychiatric practice (pp. 3446). NY: Cambridge University Press.

Danion, J. M., Kauffmann-Muller, F., Grange, D., Zimmerman, M. A., \& Greth, P. (1995). Affective valence of words, explicit and implicit memory in clinical depression. Journal of Affective Disorders, 34, 227-234.

Davidson, R. J., Pizzagalli, D., Nitschke, J. B., \& Putnam, K. (2002). Depression: Perspectives from affective neuroscience. Annual Review of Psychology, 53, 545-574.

Dawes, R. M. (2001). Everyday irrationality: How pseudoscientists, lunatics, and the rest of us systematically fail to think rationally. Boulder, CO: Westview Press.

De Ruiter, C., \& Brosschot, J. F. (1994). The emotional Stroop interference effect in anxiety: Attentional bias or cognitive avoidance? Behaviour Research \& Therapy, 32, 315-319.

Deldin, P. J., Keller, J., Gergen, J. A., \& Miller, G. A. (2001). Cognitive bias and emotion in neuropsychological models of depression. Cognition \& Emotion, 15, 787 - 802.

Denny, E. B., \& Hunt, R. R. (1992). Affective valence and memory in depression: Dissociation of recall and fragment completion. Journal of Abnormal Psychology, 101, 575-580. 
Dozois, D. J. A., \& Dobson, K. S. (2001). Information processing and cognitive organization in unipolar depression: Specificity and comorbidity issues. Journal of Abnormal Psychology, 110, 236 - 246.

Dozois, D. J. A., Dobson, K. S., \& Ahnberg, J. L. (1998). A psychometric evaluation of the Beck Depression Inventory-II. Psychological Assessment, 10, 83-89.

Ehrlichman, H., \& Halpern, J. N. (1988). Affect and memory: Effects of pleasant and unpleasant odors on retrieval of happy and unhappy memories. Journal of Personality \& Social Psychology, 55, 769-779.

Ellis, H. C., \& Moore, B. A. (1999). Mood and Memory. In T. Dalgleish \& M. J. Power (Eds.), Handbook of cognition and emotion (pp. 193210). Chichester: John Wiley \& Sons.

Endler, N. S., Cox, B. J., Parker, J. D., \& Bagby, R. M. (1992). Self-reports of depression and state-trait anxiety: Evidence for differential assessment. Journal of Personality \& Social Psychology, 63, 832-838.

Enns, M. W., Cox, B. J., Parker, J. D. A., \& Guertin, J. E. (1998). Confirmatory factor analysis of the Beck Anxiety and Depression in patients with major depression. Journal of Affective Disorders, 47, 195-200.

Erdfelder, E., Faul, F., \& Buchner, A. (1996). GPOWER: A general power analysis program. Behavior Research Methods, Instruments, \& Computers, 28, 1-11.

Eysenck, M. W., \& Byrne, A. (1994). Implicit memory bias, explicit memory bias, and anxiety. Cognition \& Emotion, 8, 415 - 431.

Eysenck, M. W., MacLeod, C., \& Mathews, A. (1987). Cognitive functioning and anxiety. Psychological Research, 49, 189-195. 
Feske, U., \& Chambless, D. L. (1995). Cognitive behavioral versus exposure only treatment for social phobia: A meta-analysis. Behavior Therapy, 26, 695-720.

Flett, G. L., Vredenburg, K., \& Krames, L. (1997). The continuity of depression in clinical and nonclinical samples. Psychological Bulletin, 121, 395-416.

Fogarty, S. J., \& Hemsley, D. R. (1983). Depression and the accessibility of memories: A longitudinal study. British Journal of Psychiatry, 142, 232-237.

Forgas, J. P., Bower, G. H., \& Krantz, S. E. (1984). The influence of mood on perceptions of social interactions. Journal of Experimental Social Psychology, 20, 497-513.

Free, M. L. (1999). Cognitive therapy in groups: Guidelines and resources for practice. NY: John Wiley \& Sons.

Gilhooly, K. J., \& Green, A. J. K. (1988). The use of memory by experts and novices. In A. M. Colley \& J. R. Beech (Eds.), Cognition and action in skilled behaviour. Advances in psychology (Vol. 55, pp. 379-395). Oxford, England: North-Holland.

Goddard, L., Dritschel, B., \& Burton, A. (1996). Role of autobiographical memory in social problem solving and depression. Journal of Abnormal Psychology, 105, 609-616.

Gotlib, I. H., \& Cane, D. B. (1987). Construct accessibility and clinical depression: A longitudinal investigation. Journal of Abnormal Psychology, 96, 199-204. 
Gotlib, I. H., \& Hammen, C. L. (1992). Psychological aspects of depression: Toward a cognitive-interpersonal integration. New York, NY: John Wiley \& Sons.

Gotlib, I. H., \& Krasnoperova, E. (1998). Biased information processing as a vulnerability factor for depression. Behavior Therapy, 29, 603617.

Gotlib, I. H., Lewinsohn, P. M., \& Seeley, J. R. (1995). Symptoms versus a diagnosis of depression: Differences in psychosocial functioning. Journal of Consulting \& Clinical Psychology, 63, 90-100.

Gotlib, I. H., \& MacLeod, C. (1997). Information processing in anxiety and depression: A cognitive-developmental perspective. In J. A. Burack \& J. T. Enns (Eds.), Attention, development, and psychopathology (pp. 350-378). New York: The Guilford Press.

Gotlib, I. H., \& McCann, C. D. (1984). Construct accessibility and depression: An examination of cognitive and affective factors. Personality \& Social Psychology, 47, 427-439.

Gotlib, I. H., McLachlan, A. L., \& Katz, A. N. (1988). Biases in visual attention in depressed and nondepressed individuals. $\underline{\text { Cognition \& }}$ Emotion, 2, 185-200.

Gotlib, I. H., \& Neubauer, D. L. (2000). Information-processing approaches to the study of cognitive biases in depression. In S. L. Johnson \& A. M. Hayes (Eds.), Stress, coping, and depression (pp. 117-143). Mahwah, NJ: Lawrence Erlbaum Associates. 
Gotlib, I. H., Ranganath, C., \& Rosenfeld, J. P. (1998). Frontal EEG alpha asymmetry, depression, and cognitive functioning. $\underline{\text { Cognition \& }}$ Emotion, 12, 449-478.

Gotlib, I. H., Roberts, J. E., \& Gilboa, E. (1996). Cognitive interference in depression. In I. G. Sarason \& G. R. Pierce (Eds.), Cognitive interference: Theories, methods, and findings. The LEA series in personality and clinical psychology (pp. 347-377). Mahwah, NJ: Lawrence Erlbaum Associates.

Gray, J. A. (1982). Precis of The Neuropsychology of Anxiety: An Enquiry into the Functions of the Septo-Hippocampal System. Behavioral \& Brain Sciences, 5, 469-534.

Haaga, D. A., \& Solomon, A. (1993). Impact of Kendall, Hollon, Beck, Hammen, and Ingram (1987) on treatment of the continuity issue in "depression" research. Cognitive Therapy \& Research, 17, 313-324.

Halberstadt, J. B., Niedenthal, P. M., \& Kushner, J. (1995). Resolution of lexical ambiguity by emotional state. Psychological Science, 6 , 278-282.

Hammen, C., Miklowitz, D. J., \& Dyck, D. G. (1986). Stability and severity parameters of depressive self-schema responding. Journal of Social \& Clinical Psychology, 4, 23-45.

Herrmann, C. (1997). International experiences with the hospital anxiety and depression scale: A review of validation data and clinical results. Journal of Psychosomatic Research, 42, 17-41.

Hill, A. B., \& Dutton, F. (1989). Depression and selective attention to self-esteem threatening words. Personality \& Individual Differences, $\underline{10}, 915-917$. 
Hill, A. B., \& Knowles, T. H. (1991). Depression and the "emotional" Stroop effect. Personality \& Individual Differences, 12, 481-485.

Horowitz, L. M., Nelson, K. L., \& Person, E. A. (1997). Using empirical research findings to develop a behavioral measure of depression: A proposed direction for future research. In H. H. Strupp \& L. M. Horowitz (Eds.), Measuring patient changes in mood, anxiety, and personality disorders: Toward a core battery. (pp. 339-368). Washington, DC: American Psychological Association.

Hsu, L. M. (2000). Effects of directionality of significance tests on the bias of accessible effect sizes. Psychological Methods, 5, 333-342.

Hunt, M. M. (1993). The story of psychology. New York: Doubleday \& Co.

Iaccino, J. F. (1993). Left brain-right brain differences: Inquiries, evidence, and new approaches. Hillsdale, NJ: Lawrence Erlbaum Associates.

Ilardi, S. S., \& Craighead, W. E. (1999). The relationship between personality pathology and dysfunctional cognitions in previously depressed adults. Journal of Abnormal Psychology, 108, 51-57.

Ilardi, S. S., \& Feldman, D. (2001). The cognitive neuroscience paradigm: A unifying metatheoretical framework for the science and practice of clinical psychology. Journal of Clinical Psychology, 57, 1067-1088.

Ingram, R. E., \& Hamilton, N. A. (1999). Evaluating precision in the social psychological assessment of depression: Methodological considerations, issues, and recommendations. Journal of Social \& Clinical Psychology, 18, 160-180. 
Jacobson, N. S., Martell, C. R., \& Dimidjian, S. (2001).

Behavioral activation treatment for depression: Returning to contextual roots. Clinical Psychology-Science \& Practice, 8, 255-270.

Jenkins, J. J., Russell, W. A., \& Suci, G. J. (1958). An atlas of semantic profiles for 360 words. American Journal of Psychology, 71, 688-699.

Jenkins, W., \& McDowall, J. (2001). Implicit memory and depression: An analysis of perceptual and conceptual processes. $\underline{\text { Cognition \& }}$ Emotion, 15, 803 - 812.

Jensen, A. R. (1998). The g factor: The science of mental ability. Westport, CT: Praeger Publishers/Greenwood Publishing Group.

Johnson, E. J., \& Tversky, A. (1983). Affect, generalization, and the perception of risk. Journal of Personality \& Social Psychology, 45, 20-31.

Johnson, M. K., \& Multhaup, K. S. (1992). Emotion and MEM. In S.-A. Christianson (Ed.), The handbook of emotion and memory: Research and theory (pp. 33-66). Hillsdale: Lawrence Erlbaum Associates.

Kendall, P. C., Hollon, S. D., Beck, A. T., Hammen, C. L., \& Ingram, R. E. (1987). Issues and recommendations regarding use of the Beck Depression Inventory. Cognitive Therapy \& Research, 11, 289-299.

Kennedy, B. L., Schwab, J. J., Morris, R. L., \& Beldia, G. (2001). Assessment of state and trait anxiety in subjects with anxiety and depressive disorders. Psychiatric Quarterly, 72, 263-276.

Klieger, D. M., \& Cordner, M. D. (1990). The Stroop task as measure of construct accessibility in depression. Personality \& Individual Differences, 11, 19-27. 
Knight, L. (Ed.). (1993). Collins English dictionary and thesaurus. Glasgow: HarperCollins.

Krantz, S., \& Hammen, C. L. (1979). Assessment of cognitive bias in depression. Journal of Abnormal Psychology, 88, 611-619.

Kucera, H., \& Francis, W. N. (1967). Computational analysis of present-day American English. Providence: Brown University Press.

Kuiper, N. A., \& MacDonald, M. R. (1983). Schematic processing in depression: The self-based consensus bias. Cognitive Therapy \& Research, 7, 469-484.

Kumari, V., Hemsley, D. R., Cotter, P. A., Checkley, S. A., \& Gray, J. A. (1998). Haloperidol-induced mood and retrieval of happy and unhappy memories. Cognition \& Emotion, 12, 497-508.

Kuyken, W., \& Dalgleish, T. (1995). Autobiographical memory and depression. British Journal of Clinical Psychology, 34, 89-92.

Kyrios, M., \& Iob, M. A. (1998). Automatic and strategic processing in obsessive-compulsive disorder: Attentional bias, cognitive avoidance or more complex phenomena? Journal of Anxiety Disorders, 12, 271292.

Lang, A. J., \& Craske, M. G. (1997). Information processing in anxiety and depression. Behaviour Research \& Therapy, 35, 451-455.

Lawson, C., \& MacLeod, C. (1999). Depression and the interpretation of ambiguity. Behaviour Research \& Therapy, 37, 463-474.

Lecrubier, Y. (1998). Is depression under-recognised and undertreated? International Clinical Psychopharmacology, 13, S3-S6. 
LeDoux, J. E. (1995). Emotion: Clues from the brain. Annual Review of Psychology, 46, 209-235.

LeDoux, J. E. (1996). The emotional brain: The mysterious underpinnings of emotional life. NY: Simon \& Schuster.

LeDoux, J. E. (2000). Emotion Circuits in the Brain. Annual Review of Neuroscience, 23, 155-184.

Leventhal, L. (1999). Updating the debate on one- versus twotailed tests with the directional two-tailed test. Psychological Reports, 84, 707718.

Lewinsohn, P. M., Solomon, A., Seeley, J. R., \& Zeiss, A. (2000). Clinical implications of "subthreshold" depressive symptoms. Abnormal Psychology, 109, 345-351.

Lilienfeld, S. O. (1995). Seeing both sides: Classic controversies in abnormal psychology. Pacific Grove, CA: Brooks/Cole.

Lipsey, M. W. (1998). Design sensitivity: Statistical power for applied experimental research. In L. Bickman \& D. J. Rog (Eds.), $\underline{\text { Handbook of }}$ applied social research methods (pp. 39-68). Thousand Oaks, CA: Sage Publications.

Lovibond, P. F., \& Lovibond, S. H. (1995). The structure of negative emotional states: Comparison of the Depression Anxiety Stress Scales (DASS) with the Beck Depression and Anxiety Inventories. Behaviour Research \& Therapy, 33, 335-343.

Mackinger, H. F., Pachinger, M. M., Leibetseder, M. M., \& Fartacek, R. R. (2000). Autobiographical memories in women remitted from major depression. Journal of Abnormal Psychology, 109, 331-334. 
MacLeod, A. K., Andersen, A., \& Davies, A. (1994). Self-ratings

of positive and negative affect and retrieval of positive and negative affect memories. Cognition \& Emotion, 8, 483-488.

MacLeod, C. (1990). Mood disorders and cognition. In M. W. Eysenck (Ed.), Cognitive psychology: An international review (pp. 9-56). Oxford: John Wiley \& Sons.

MacLeod, C., \& Hagan, R. (1992). Individual differences in the selective processing of threatening information, and emotional responses to a stressful life event. Behaviour Research \& Therapy, 30, 151-161.

MacLeod, C., Mathews, A., \& Tata, P. (1986). Attentional bias in emotional disorders. Journal of Abnormal Psychology, 95, 15-20.

MacLeod, C., \& Mathews, A. M. (1991). Cognitive-experimental approaches to the emotional disorders. In P. R. Martin (Ed.), $\underline{\text { Handbook of }}$ behavior therapy and psychological science: An integrative approach (pp. 116150). Elmsford, NY: Pergamon Press.

MacLeod, C., \& Rutherford, E. M. (1998). Automatic and strategic cognitive biases in anxiety and depression. In K. Kirsner \& C. Speelman (Eds.), Implicit and explicit mental processes (pp. 233-254). Mahwah, NJ: Lawrence Erlbaum Associates.

MacLeod, C. M. (1991). Half a century of research on the Stroop effect: An integrative review. Psychological Bulletin, 109, 163-203.

Macmillan, M. (1991). Freud evaluated : the completed arc. Amsterdam: North-Holland. 
Mandal, M. K., Asthana, H. S., Pandey, R., \& Sarbadhikari, S. (1996). Cerebral laterality in affect and affective illness: A review. Journal of Psychology, 130, 447-459.

Mathews, A. (1990). Why worry? The cognitive function of anxiety. Behaviour Research \& Therapy, 28, 455-468.

Mathews, A., \& MacLeod, C. (1985). Selective processing of threat cues in anxiety states. Behaviour Research \& Therapy, 23, 563-569.

Mathews, A., \& MacLeod, C. (2002). Induced processing biases have causal effects on anxiety. Cognition \& Emotion, 16, 331-354.

Mathews, A., Mogg, K., May, J., \& Eysenck, M. (1989). Implicit and explicit memory bias in anxiety. Journal of Abnormal Psychology, 98, 236240.

Mathews, A., Richards, A., \& Eysenck, M. (1989). Interpretation of homophones related to threat in anxiety states. Journal of Abnormal Psychology, 98, 31-34.

Mathews, A., Ridgeway, V., \& Williamson, D. A. (1996). Evidence for attention to threatening stimuli in depression. Behaviour Research \& Therapy, 34, 695-705.

Matt, G. E., Vazquez, C., \& Campbell, W. K. (1992). Moodcongruent recall of affectively toned stimuli: A meta-analytic review. $\underline{\text { Clinical }}$ Psychology Review, 12, 227-255.

Mayo, P. R. (1983). Personality traits and the retrieval of positive and negative memories. Personality \& Individual Differences, 4, 465-471.

Mayo, P. R. (1989). A further study of the personality-congruent recall effect. Personality \& Individual Differences, 10, 247-252. 
McCabe, S. B., \& Gotlib, I. H. (1995). Selective attention and clinical depression: Performance on a deployment-of-attention task. Journal of Abnormal Psychology, 104, 241-245.

McCabe, S. B., \& Toman, P. E. (2000). Stimulus exposure duration in a deployment-of-attention task: Effects on dysphoric, recently dysphoric, and nondysphoric individuals. Cognition \& Emotion, 14, 125-142.

McNally, R. J., Lasko, N. B., Macklin, M. L., \& Pitman, R. K. (1995). Autobiographical memory disturbance in combat-related posttraumatic stress disorder. Behaviour Research \& Therapy, 33, 619-630.

McNeil, D. W., Tucker, P., Miranda Jr, R., Lewin, M. R., \& Nordgren, J. C. (1999). Response to depression and anxiety Stroop stimuli in posttraumatic stress disorder, obsessive-compulsive disorder, and major depressive disorder. Journal of Nervous \& Mental Disease, 187, 512-516.

Meehl, P. E. (1990). Why summaries of research on psychological theories are often uninterpretable. Psychological Reports, 66, 195-244.

Merckelbach, H., de Jong, P., \& Muris, P. (1990). Conjugate lateral eye movements, cerebral dominance, and anxiety. In P. J. D. Drenth \& J. A. Sergeant (Eds.), European perspectives in psychology, Vol. 2: Clinical, health, stress and anxiety, neuropsychology, psychophysiology. (pp. 369-379). New York: John Wiley \& Sons.

Merckelbach, H., Muris, P., \& Horselenberg, R. (1996). Correlates of overgeneral memories in normal subjects. Behavioural \& Cognitive Psychotherapy, 24, 109-115. 
Merckelbach, H., Muris, P., Pool, K., \& De Jong, P. J. (1996).

Reliability and validity of a paper-and-pencil test measuring hemisphere preference. European Journal of Personality, 10, 221-231.

Mineka, S., Watson, D., \& Clark, L. A. (1998). Comorbidity of anxiety and unipolar mood disorders. Annual Review of Psychology, 49, 377412.

Mogg, K., Bradley, B. P., Miller, T., Potts, H., Glenwright, J., \& Kentish, J. (1994). Interpretation of homophones related to threat: Anxiety or response bias effects? Cognitive Therapy \& Research, 18, 461-477.

Mogg, K., Bradley, B. P., \& Williams, R. (1995). Attentional bias in anxiety and depression: The role of awareness. British Journal of Clinical Psychology, 34, 17-36.

Mogg, K., Mathews, A., May, J., Grove, M., Eysenck, M., \& Weinman, J. (1991). Assessment of cognitive bias in anxiety and depression using a colour perception task. Cognition \& Emotion, 5, 221-238.

Moore, R. G., Watts, F. N., \& Williams, J. M. (1988). The specificity of personal memories in depression. British Journal of Clinical Psychology, 27, 275-276.

Nunnally, J. C., \& Bernstein, J. H. (1994). Psychometric theory (3rd ed.). New York: McGraw Hill.

Oatley, K., \& Johnson-Laird, P. N. (1987). Towards a cognitive theory of emotions. Cognition \& Emotion, 1, 29-50.

Osman, A., Barrios, F. X., Aukes, D., Osman, J. R., \& Markway, K. (1993). The Beck Anxiety Inventory: Psychometric properties in a community population. Journal of Psychopathology \& Behavioral Assessment, 15, 287 - 297. 
Overall, J. E. (1990). Tests of one-sided versus two-sided hypotheses in placebo-controlled clinical trials. Neuropsychopharmacology, 3 , 233-235.

Ratcliff, R. (1993). Methods for dealing with reaction time outliers. Psychological Bulletin, 114, 510-532.

Richards, A., \& Whittaker, T. M. (1990). Effects of anxiety and mood manipulation in autobiographical memory. British Journal of Clinical Psychology, 29, 145-153.

Roediger, H. L., \& McDermott, K. B. (1992). Depression and implicit memory: A commentary. Journal of Abnormal Psychology, 101, 587591.

Rolls, E. T., \& Treves, A. (1998). Neural networks and brain function. NY: Oxford University Press.

Rude, S. S., Wenzlaff, R. M., Gibbs, B., Vane, J., \& Whitney, T. (2002). Negative processing biases predict subsequent depressive symptoms. Cognition \& Emotion, 16, 423-440.

Ruiz-Caballero, J. A., \& Gonzalez, P. (1994). Implicit and explicit memory bias in depressed and nondepressed subjects. Cognition \& Emotion, 8, 555-569.

Ruscio, J., \& Ruscio, A. M. (2000). Informing the continuity controversy: A taxometric analysis of depression. Journal of Abnormal Psychology, 109, 473-487.

Rusting, C. L. (1999). Interactive effects of personality and mood on emotion-congruent memory and judgment. Journal of Personality \& Social Psychology, 77, 1073-1086. 
Schwartz, G. E., Davidson, R. J., \& Goleman, D. J. (1978).

Patterning of cognitive and somatic processes in the self-regulation of anxiety:

Effects of meditation versus exercise. Psychosomatic Medicine, 40, 321-328.

Schwarz, N., \& Clore, G. L. (1983). Mood, misattribution, and judgments of well-being: Informative and directive functions of affective states. Journal of Personality \& Social Psychology, 45, 513-523.

Scott, K. M., Mogg, K., \& Bradley, B. P. (2001). Masked

semantic priming of emotional information in subclinical depression. $\underline{\text { Cognitive }}$ Therapy \& Research, 25, 505-524.

Segal, Z. V., Lau, M. A., \& Rokke, P. D. (1999). Cognition and emotion research and the practice of cognitive-behavioural therapy. In T. Dalgleish \& M. J. Power (Eds.), Handbook of cognition and emotion (pp. 705726). Chichester, England: John Wiley \& Sons.

Shelton, R. C., Hollon, S. D., Purdon, S. E., \& Loosen, P. T. (1991). Biological and psychological aspects of depression. Behavior Therapy, 22, 201-228.

Sidley, G. L., Whitaker, K., Calam, R. M., \& Wells, A. (1997). The relationship between problem-solving and autobiographical memory in parasuicde patients. Behavioural \& Cognitive Psychotherapy, 25, 195-202. Snaith, R. P. (1992). Availability of the Hospital Anxiety and Depression (HAD) scale. British Journal of Psychiatry, 161, 422.

Spearman, C. (1910). Correlation calculated from faulty data. British Journal of Psychology, 3, 271-295. 
Spielberger, C. D., Gorsuch, R. L., Lushene, R., Vagg, P. R., \& Jacobs, G. A. (1983). Manual for the State-Trait Anxiety Inventory. Palo Alto, CA: Consulting Psychologists Press.

Spinhoven, P. H., Ormel, J., Sloekers, P. P. A., \& Kempen, G. I. J. M. (1997). A validation study of the Hospital Anxiety and Depression scale (HADS) in different groups of Dutch subjects. Psychological Medicine, 27, 363370.

Sweet, A. A., \& Loizeaux, A. L. (1991). Behavioral and cognitive treatment methods: A critical comparative review. Journal of Behavior Therapy \& Experimental Psychiatry, 22, 159-185.

Teasdale, J. D. (1999). Multi-level theories of cognition-emotion relations. In T. Dalgleish \& M. J. Power (Eds.), $\underline{\text { Handbook of cognition and }}$ emotion (pp. 665-681). Chichester: John Wiley \& Sons.

Teasdale, J. D., \& Barnard, P. J. (1993). Affect, cognition, and change: Re-modelling depressive thought. Hillsdale, NJ: Lawrence Erlbaum Associates.

Teasdale, J. D., \& Fogarty, S. J. (1979). Differential effects of induced mood on retrieval of pleasant and unpleasant events from episodic memory. Journal of Abnormal Psychology, 88, 248 - 257.

Teasdale, J. D., \& Russell, M. L. (1983). Differential effects of induced mood on the recall of positive, negative and neutral words. $\underline{\text { British }}$ Journal of Clinical Psychology, 22, 163-171.

Urdang, L. (1997). The Oxford Thesaurus (2nd edn.). Oxford: Clarendon Press. 
van Blakom, A. J. L. M., van Oppen, P., Vermeulen, A. W. A., van Dyck, R., Nauta, M. C. E., \& Vorst, H. M. C. (1994). A meta-analysis on the treatment of obsessive compulsive disorder: A comparison of antidepressants, behavior, and cognitive therapy. Clinical Psychology Review, 14, 359-381.

Van den Hout, M., Tenney, N., Huygens, K., Merckelbach, H., \& Kindt, M. (1995). Responding to subliminal threat cues is related to trait anxiety and emotional vulnerability: A successful replication of Macleod and Hagan (1992). Behaviour Research \& Therapy, 33, 451-454.

Vredenburg, K., Flett, G. L., \& Krames, L. (1993). Analogue versus clinical depression: A critical reappraisal. Psychological Bulletin, 113, 327-344.

Watkins, E., Teasdale, J. D., \& Williams, R. M. (2000). Decentring and distraction reduce overgeneral autobiographical memory in depression. Psychological Medicine, 30, 911-920.

Watkins, P. C., Martin, C. K., \& Stern, L. D. (2000). Unconscious memory bias in depression: Perceptual and conceptual processes. Abnormal Psychology, 109, 282-289.

Watkins, P. C., Mathews, A., Williamson, D. A., \& Fuller, R. D. (1992). Mood-congruent memory in depression: Emotional priming or elaboration? Journal of Abnormal Psychology, 101, 581-586.

Watkins, P. C., Vache, K., Verney, S. P., \& Mathews, A. (1996). Unconscious mood-congruent memory bias in depression. Journal of Abnormal Psychology, 105, 34-41. 
Watson, D., Clark, L. A., \& Tellegen, A. (1988). Development and validation of brief measures of positive and negative affect: The PANAS scales. Journal of Personality \& Social Psychology, 54, 1063-1070.

Wenzlaff, R. M., \& Bates, D. E. (1998). Unmasking a cognitive vulnerability to depression: How lapses in mental control reveal depressive thinking. Journal of Personality \& Social Psychology, 75, 1559-1571.

Wenzlaff, R. M., \& Eisenberg, A. R. (2001). Mental control after dysphoria: Evidence of a suppressed, depressive bias. Behavior Therapy, 32, 2745.

Wessel, I., Meeren, M., Peeters, F., Arntz, A., \& Merckelbach, H. (2001). Correlates of autobiographical memory specificity: The role of depression, anxiety and childhood trauma. Behaviour Research \& Therapy, 39, 409-421.

Westra, H. A., \& Kuiper, N. A. (1997). Cognitive content specificity in selective attention across four domains of maladjustment. Behaviour Research \& Therapy, 35, 349-365.

Williams, J. M., \& Broadbent, K. (1986). Autobiographical memory in suicide attempters. Journal of Abnormal Psychology, 95, 144-149.

Williams, J. M., \& Dritschel, B. H. (1988). Emotional disturbance and the specificity of autobiographical memory. Cognition \& Emotion, 2, 221234.

Williams, J. M., \& Scott, J. (1988). Autobiographical memory in depression. Psychological Medicine, 18, 689-695.

Williams, J. M. G. (1992). Autobiographical memory and emotional disorders. In S. A. Christianson (Ed.), The handbook of emotion and 
memory: Research and theory. (pp. 451-477). Hillsdale, NJ: Lawrence Erlbaum Associates.

Williams, J. M. G. (1996). Depression and the specificity of autobiographical memory. In D. C. Rubin (Ed.), Remembering our past: Studies in autobiographical memory (pp. 244-267). New York: Cambridge University Press.

Williams, J. M. G., \& Dritschel, B. (1992). Categoric and extended autobiographical memories. In M. A. Conway, D. C. Rubin, H. Spinnler \& W. A. Wagenaar (Eds.), Theoretical Perspectives on Autobiographical Memory (pp. 391 - 412). Dordrecht, The Netherlands: Kluwer Academic.

Williams, J. M. G., Mathews, A., \& MacLeod, C. (1996). The emotional Stroop task and psychopathology. Psychological Bulletin, 120, 3-24.

Williams, J. M. G., Teasdale, J. D., Segal, Z. V., \& Soulsby, J. (2000). Mindfulness-based cognitive therapy reduces overgeneral autobiographical memory in formerly depressed patients. Journal of Abnormal Psychology, 109, 150-155.

Williams, J. M. G., Watts, F. N., MacLeod, C., \& Mathews, A. (1988). Cognitive psychology and emotional disorders. Oxford, England: John Wiley \& Sons.

Williams, J. M. G., Watts, F. N., MacLeod, C., \& Mathews, A. (1997). Cognitive psychology and emotional disorders (2nd ed). Chichester: John Wiley \& Sons. 
Williams, W. H., Williams, J. M. G., \& Ghadiali, E. J. (1998). Autobiographical memory in traumatic brain injury: Neuropsychological and mood predictors of recall. Neuropsychological Rehabilitation, 8, 43 - 60.

Wing, A. M., \& Baddeley, A. D. (1980). Spelling errors in handwriting: A corpus and a distributional analysis. In U. Frith (Ed.), Cognitive processes in spelling (pp. 251 - 285). London: Academic Press.

Zarantonello, M., Slaymaker, F., Johnson, J. E., \& Petzel, T. (1984). Effects of anxiety and depression on anagram performance, ratings of cognitive interference, and the negative subjective evaluation of performance. Journal of Clinical Psychology, 40, 20-25.

Zenhausern, R. (1978). Imagery, cerebral dominance, and style of thinking: A unified field model. Bulletin of the Psychonomic Society, 12, 381384.

Zigmond, A. S., \& Snaith, R. P. (1983). The Hospital Anxiety and Depression Scale. Acta Psychiatrica Scandinavica, 67, 361-370.

Zimmerman, M., \& Coryell, W. (1987). The Inventory to Diagnose Depression (IDD): A self-report scale to diagnose major depressive disorder. Journal of Consulting \& Clinical Psychology, 55, 55-59. 